\title{
The generation and amplification of intergalactic magnetic fields in analogue laboratory experiments with high power lasers
}

\author{
G. Gregoria ${ }^{\mathrm{a}}$, B. Reville ${ }^{\mathrm{b}}$, F. Miniatic \\ ${ }^{a}$ Department of Physics, University of Oxford, Parks Road, Oxford OX1 3PU, United Kingdom \\ ${ }^{b}$ Centre for Plasma Physics, Queen's University Belfast, University Road, BT7 1NN, United Kingdom \\ ${ }^{c}$ Physics Department, Wolfgang-Pauli-Strasse 27, ETH-Zürich, CH-8093 Zürich, Switzerland
}

\begin{abstract}
The advent of high-power laser facilities has, in the past two decades, opened a new field of research where astrophysical environments can be scaled down to laboratory dimensions, while preserving the essential physics. This is due to the invariance of the equations of magneto-hydrodynamics to a class of similarity transformations. Here we review the relevant scaling relations and their application in laboratory astrophysics experiments with a focus on the generation and amplification of magnetic fields at cosmological shock waves. These arise during the collapse of protogalactic structures, resulting in the formation of high Mach number shocks in the intergalactic medium, which act as sources of vorticity in protogalaxies. The standard model for the origin of magnetic fields is via baroclinic generation from the resulting misaligned pressure and temperature gradients (the so-called Biermann battery process). While both experiment and numerical simulation have confirmed the occurrence of this mechanism at shocks, reconciling the resulting weak fields with present day observations is an un-solved problem, although it is generally accepted that turbulent motions of the weakly magnetised plasma plays a key role. Bridging the vast scale differences is a challenge both numerically and experimentally. A summary of novel laboratory experiments aimed at investigating additional processes that may shed light on these and other processes, such us turbulent amplification, resistive and collision-less plasma instabilities will be discussed in this review, particularly in relation to experiments using high power laser systems. The connection between laboratory shock waves and additional mechanisms, such as diffusive shock acceleration will be discussed. Finally, we will summarize the impact of laboratory investigation in furthering our understanding of plasma physics on super-galactic scales.
\end{abstract}

Keywords:

\section{Introduction}

This review focuses on the laboratory simulation of astrophysical environments. This is a very broad and rapidly developing field of research, with several review papers and books having already appeared on the topic [e.g., 1, 2, 3, 4]. As is frequently pointed out in these reviews, the field of laboratory astrophysics is at the foundation of astronomy and astrophysics. It addresses fundamental questions related to the astrophysical objects through the generation of controlled, reproducible conditions in terrestrial experiments, thus offering a viable complementary approach to both astrophysical observations (by providing, for example, the means of directly measuring quantities of interest not accessible by observation) and numerical calculations, overcoming limitations in resolution, numerical viscosity and potentially addressing non-linear aspects of the dynamical evolution. This approach has also proved important in the validation of simulation codes [5].

Laboratory astrophysics covers a vast range of topics, with applications arising from high-energy particle physics, nuclear physics, condensed matter physics, etc. In some cases, matter and plasma conditions which are analogous to those found in astrophysical counterparts can be directly reproduced and studied in the laboratory. In most situations, however, it is not possible to reach the exact conditions found in the astrophysical environment because the spatial, temporal, and energy scales may be outside the range of what is directly reproducible in an experiment. A different approach relies on making connections between experiments and astrophysical objects through similarity criteria. Making such a connection can only be achieved by careful examination of the relations between experimental data, 
observations of the astrophysical phenomenon being investigated experimentally, and numerical simulations of both the experiment and the astrophysical system. Here we explore this approach further, concentrating on a specific problem: laboratory astrophysics and its use to study primordial magnetogenesis and the amplification of magnetic fields by plasma processes. Clearly, this is possible (and meaningful) only if we can devise a means by which the relevant physics in a terrestrial laboratory can be related to the Universe at its largest scales. At first sight, this may be difficult to visualise as most of the parameters of interest, such as the spatial, temporal and energy scales associated to the structure formation and evolution in the Universe are clearly not reproducible in an experiment. Nevertheless, a connection between the two systems does indeed exist, provided we can show that the laboratory and astrophysical environments evolve in such a way that the governing equations are invariant under a scale transformation; this requires the corresponding spatial, density, pressure, time, and so on, values in one system to be mapped onto the other system by an appropriate combination of multiplicative constants. This is an example of what we call a similarity relation. This similarity can be obtained via plasma-fluid equations $[6,7,8,9,10,11]$, or even at the kinetic level $[12,13]$ under some conditions.

\section{Astrophysical context}

It is generally accepted that magnetic fields are present almost everywhere in the universe, from stars and planets to the interstellar medium of galaxies $[14,15]$, to the hot intracluster medium of galaxy clusters $[16,17,18]$ and even in the vast cosmic voids $[19,20,21]$. The fundamental question raised by these observations is clearly the origin of the magnetic field. That dynamo action, i.e. the generation of magnetic field through the hydrodynamic motion of a conducting fluid, is the principal mechanism is in little doubt. The hypothesis of hydrodynamic dynamo was first proposed in 1919 by Larmor to explain the origin of the Earth's magnetic field, but the process turned out highly non trivial and required decades of study to reach maturity [22]. Having expanded in scope, it remains to date an active field of research [23]. The equation for the magnetic field evolution in a highly conducting fluid are derived starting from Faraday's induction equation,

$$
\frac{\partial \mathbf{B}}{\partial t}=-\nabla \times \mathbf{E},
$$

with the electric field obtained from Ohm's law. In the simplest model, the latter reads

$$
\mathbf{J}=\sigma \mathbf{E}^{\prime}=\sigma(\mathbf{E}+\mathbf{u} \times \mathbf{B})
$$

where $\mathbf{E}^{\prime}$ is the electric field in the local rest frame of a given fluid element, with $\sigma$ the conductivity. Combining with Ampère's law,

$$
\mathbf{J}=\frac{1}{\mu_{0}} \nabla \times \mathbf{B}
$$

where the displacement current has been neglected. Assuming constant conductivity, the induction equation becomes

$$
\frac{\partial \mathbf{B}}{\partial t}=\nabla \times(\mathbf{u} \times \mathbf{B})+\frac{\eta}{\mu_{0}} \nabla^{2} \mathbf{B}
$$

where the first term on the RHS represents the effect of the induced electric field, while the second term represents Ohmic diffusion with $\eta=1 / \sigma$ the magnetic diffusivity. Equation (4) contains the effect of hydrodynamic dynamo. Note from the linearity of $\mathbf{B}$ in equation (4), it is immediately clear that hydrodynamic dynamo only works if a non vanishing magnetic field already exists. For this reason the origin of magnetic field in astrophysical environment is understood as the result of two basic steps [24, 23]: ( $i$ ) the generation of a seed and (ii) the amplification by the hydromagnetic mechanism. Two classes of hydromagnetic dynamo are defined, namely large scale and small scale dynamo, which, depending on the characteristic of the flow (helicity), determine whether a magnetic field is amplified on scales larger or smaller than the outer scale of the turbulence.

In the following we shall focus on the generation of magnetic seeds and the amplification of magnetic field by the small scale dynamo mechanism in a turbulent flow in the absence of large scale helicity. In addition, we restrict our attention to extragalactic environments, in particular the intergalactic medium in its various forms, from the 
relatively cold supersonic flows characterising the gravitationally induced mass accretion onto forming cosmic structures [25] such as galaxies, galaxy filaments and clusters, to the turbulent conditions of hot gas inside those forming structures [26, 27, 28, 29, 30].

The flow inside cosmic structures produced by gravitational collapse, is characterised by a high Reynolds number, $R e=u L / v$, where $L$ and $u$ are the characteristic spatial and velocity scale of the system, while $v$ is the kinematic viscosity. In addition, the magnetic Reynolds number $R m=\mu_{0} u L / \eta$ is also expected to be large. The value of the magnetic Prandtl number, $P m=\mu_{0} v / \eta$, in the intergalactic space is not clear. Spitzer's value taken at face value gives a very large $P m$ number. However, in a high $\beta$ (defined as the ratio of thermal to magnetic pressures) plasma, the particle mean free path is likely determined by plasma micro instabilities $[31,32,33]$ instead of Coulomb collisions, which restricts $P m$ to values not vastly different from unity. In any case, the turbulent velocity field is to a good approximation homogeneous, isotropic and non-helical [28, 29], so that the relevant magnetic field amplification mechanism is the small scale or turbulent dynamo. According to the current standard picture, the amplification by the turbulent dynamo can be summarised in three basic steps $[34,35,36]$. First, when the initial magnetic field is of vanishing strength, magnetic energy grows exponentially, at a rate $\gamma=\sqrt{R e} / 30 \tau_{L}$, where $\tau_{L}=L / u_{L}$ is the eddy turnover time, i.e., the ratio of the turbulent outer scale $L$ to the turbulent velocity dispersion at that scale. This phase is however, short lived, and terminates when the magnetic energy reaches approximate equipartition with the kinetic energy at the viscous scale, i.e., at time $\sim \tau_{L} / \sqrt{R e}$. At this point the magnetic energy stops growing at and below the viscous scales where magnetic tension has become sufficiently strong to prevent bending of magnetic field lines. Nevertheless, the magnetic energy continues to grow, as the Alfvén scale $L_{A}$, which marks equipartition between magnetic and kinetic energy, becomes increasingly larger than the viscous scale. For Kolmogorov's turbulence, $L_{A} \equiv v_{A}^{3} / C^{3 / 2} \epsilon_{\text {turb }}$, with $\epsilon_{\text {turb }}$ the turbulent dissipation rate and $v_{A}=B / \sqrt{\mu_{0} \rho}$ is the Alfvén speed and $C$ a Kolmogorov's constant. This second stage can be described by the conversion of turbulent kinetic energy into magnetic energy at the rate corresponding to a fraction of the turbulent dissipation rate. For a constant turbulent dissipation rate the growth of magnetic energy is no longer exponential, but rather is linear. Numerical efforts have been made to estimate the efficiency of the turbulent dynamo mechanism in converting kinetic to magnetic energy in astrophysical environments, assuming an extrapolation to the huge $R e$ of astrophysical flows, with some authors claiming universal values of 4-5\% [37]. Finally, the amplification saturates when magnetic energy reaches equipartition with the turbulent kinetic energy on scales of order $\lesssim L$, i.e., a fraction of the turbulent outer scale.

Note that saturation is believed to be easily achieved whenever the dynamical timescale of the system is much shorter than the age of the universe, like for example in the case of stars, compact objects, active nuclei or even the interstellar medium. This is most likely not the case, however, in the intergalactic medium [38]. Hence the efficiency of turbulent dynamo in converting kinetic to magnetic to energy plays an important role in this case. From the above discussion it is instead clear that the strength of the initial seed is not particularly important. Seeds of different strength only produce a logarithmic delay in the evolution of the magnetic energy. Therefore, of the several mechanisms that have been proposed in the literature [see, 39, for a review] we select a few examples of particular importance in astrophysics that have been reproduced in the laboratory. These include the Biermann's battery process, Weibel's instability and the resistive mechanism induced by return currents.

The Biermann battery mechanism is of particular interest here because of the ubiquitous presence of shocks in the large scale structure of the universe. These shocks appear as cosmic structures collapse under gravitational instability. The resulting shocks have high Mach number and the inevitable geometrical asymmetries provide the misalignment between pressure and density gradients [40], i.e. the baroclynic conditions that enable the Biermann battery effect [41]. The interesting part is that this process inevitably precedes the formation of galaxies, so even in the absence of a primordial field galaxies are born magnetised. In addition, despite its importance, the Biermann battery effect has not been tested experimentally until very recently for baroclynic conditions generated by shock waves. The Weibel instability can also be produced at shocks [42, 43], which makes it interesting for the same reasons as above. However, despite its much stronger seed value, the magnetic field is generated on microscopic scales [44, 45, 46], which is prone to fast resistive dissipation. Finally, the resistive current-driven mechanism of [47] is arguably the only astrophysical process that produces sizeable magnetic seeds throughout intergalactic space. Unlike the case of collapsed structures such as galaxies and galaxy clusters, dynamo action is most likely very inefficient in cosmic voids where the flow is expanding and any turbulence would quickly dye out. Thus the value of the magnetic field 
observed in this environment reflects the value of the original seed and tell us about the generation process. ${ }^{1}$ The current estimates accounting for variability of the astronomical (gamma-ray) sources suggest $B \approx 10^{-18} \mathrm{G}$, which is consistent with analytic and numerical predictions for the resistive mechanism [47, 48]. The Biermann battery and Weibel instability on the other hand, are unlikely to operate in the voids, but rather only inside collapsed structures such as filaments and clusters. More exotic processes have been theorised in the early universe, however, a successful theory would need to explain magnetic field in voids if they turned out much larger than mentioned above.

\section{Mechanisms for the generation of seed fields}

\subsection{Generalized Ohm's law and the Biermann Battery}

Inspection of Faraday's law, equation (1), generation of magnetic field in an unmagnetised plasma is produced only if the electric field is not purely electrostatic, i.e. it cannot be described by a scalar potential $\phi$ alone, but must include a vector potential A:

$$
E=-\nabla \phi+\frac{\partial \mathbf{A}}{\partial t}
$$

Clearly only the second term contributes to Faraday's law. If the magnetic field is initially zero, any source term must clearly be independent of the field itself. To see which terms can make such a contribution to the electric field in general, requires us to revisit the Ohm's law. Starting from the equation of motion of the electron fluid, and neglecting the inertial and gravitational terms due to the smallness of the electron mass, one obtains a modified Ohm's law reading

$$
0=-n_{e} e\left(\mathbf{E}+\mathbf{v}_{e} \times \mathbf{B}\right)-\nabla p_{e}+\mathbf{F}_{e, i}, \quad \rightarrow \quad \mathbf{E}=-\mathbf{u} \times \mathbf{B}+\frac{1}{e n_{e}} \mathbf{J} \times \mathbf{B}-\frac{\nabla p_{e}}{e n_{e}}+\eta \mathbf{J},
$$

where $p_{e}, n_{e}, \mathbf{v}_{e}$ are the electron fluid pressure, density and velocity, $e$ the elementary charge, $\mathbf{J} \approx n_{e} e\left(\mathbf{u}-\mathbf{v}_{e}\right)$ the total current density with $\mathbf{u}$ the mass velocity, and $\mathbf{F}_{e, i}=e n_{e} \eta \mathbf{J}$ the collisional friction term responsible for the electric resistivity [49]. Comparing to Eq. (2), we see that two additional terms contributing to the electric field arise, the $\mathbf{J} \times \mathbf{B}$ term, known as the Hall term, and the $\nabla p_{e}$ due to the electron fluid's response to local pressure gradients. Comparing the relative magnitudes of the first and second terms on the right hand side, we see that the Hall term can be safely neglected on length scales $L \gg(c / u)\left(\omega_{\mathrm{ce}} / \omega_{\mathrm{pe}}\right) c / \omega_{\mathrm{pe}} \sim \beta_{\mathrm{e}}^{-1 / 2} c / \omega_{\mathrm{pe}}$, where $\omega_{\mathrm{ce}}, \omega_{\mathrm{pe}}$ and $\beta_{\mathrm{e}}$ are the electron cyclotron frequency, plasma frequency and plasma beta respectively. This limit applies for mosts cases of interest in this review. In any case, if the magnetic field is initially zero, this term makes no contribution to the generation of magnetic field.

We now turn our attention to the pressure gradient term. Again, if the medium is unmagnetised, and ignoring collisions for now, Ohm's law is simply

$$
\mathbf{E}=-\frac{\nabla p_{e}}{e n_{e}}
$$

Now, if the electron fluid is barotropic, i.e.. $n_{e}=n_{e}\left(p_{e}\right)$, it is straightforward to show that the electric field can be expressed as the gradient of a scalar field, i.e. there exists a balance between an electrostatic field and the electron pressure gradient, with no net flow of current, and consequently no source of magnetic field $(\partial \mathbf{A} / \partial t=0)$. However, this will not always be the case. In particular, if the density gradient is not aligned with the pressure gradient, it is immediately apparent that the electric field will cease to be curl-free. Thus, on sufficiently short times, an electron current can now flow in the plasma that will not be balanced by a corresponding motion of the ions (on account of their larger inertia), which indeed results in the appearance of a time dependent magnetic field ${ }^{2}$

$$
\frac{\partial \mathbf{B}}{\partial t}=\nabla \times\left(\frac{\nabla p_{e}}{e n_{e}}\right)=\frac{\nabla p_{e} \times \nabla n_{e}}{e n_{e}^{2}} .
$$

Indeed it is this latter effect, the inertia of the ions, that is key to providing the energy source for the magnetic field in this mechanism. Considering the source term for internal energy, $-\mathbf{J} \cdot \mathbf{E} \approx\left(\mathbf{u}-\mathbf{v}_{e}\right) \cdot \nabla p_{e}$, it is evident that the magnetic

\footnotetext{
${ }^{1}$ Of course since the seed is generated at a cosmological redshift, $z>0$, it would be diluted with respect to the original value by a factor $(1+z)^{2}$, which is trivial to account for.

${ }^{2}$ Note, using $p_{e}=n_{e} k T_{e}$ this reduces to the $\nabla n \times \nabla T$ form more familiar to laser plasma community [50].
} 
field extracts energy from the plasma simply due to the difference in the rates at which the electron pressure gradient does work on the different species in the background plasma.

To complete our derivation of the Biermann battery equations, we re-introduce the magnetic field and collisions. Considering a collisional plasma, it is reasonable to assume thermodynamic equilibrium such that the electron pressure, in a charge neutral plasma with $n_{e}=Z n_{i}$ (where $Z$ the ion charge and $n_{i}$ the ion density) can be re-expressed in terms of the total pressure, $p_{e}=Z p /(1+Z)$. Again taking constant and uniform resistivity, and using Ampére's law to remove the current, the induction equation now reads:

$$
\frac{\partial \mathbf{B}}{\partial t}=\nabla \times(\mathbf{u} \times \mathbf{B})+\frac{\eta}{\mu_{0}} \nabla^{2} \mathbf{B}+\frac{m_{i}}{e(1+Z)} \frac{\nabla p \times \nabla \rho}{\rho^{2}},
$$

with $m_{i}$ the mass of the dominant ion species. This equation is the same as (4), but with an additional term, the Biermann battery, that generates a magnetic field when pressure and density gradients are misaligned.

There are two scenarios where the Biermann battery is thought to play a crucial role in proto-galactic field generation. One associated with structure formation shocks [15] and the other with ionisation fronts produced during cosmic reionization [51, 52]. It is possible to estimate the magnitude of the seed fields produced via the Biermann mechanism in structure formation shocks if we exploit its parallel with the generation of vorticity, $\boldsymbol{\Omega}=\nabla \times \mathbf{u}$ [41]. Taking the curl of the Navier-Stokes equation produces an equation for the vorticity

$$
\frac{\partial \mathbf{\Omega}}{\partial t}=\nabla \times(\mathbf{u} \times \mathbf{\Omega})+v \nabla^{2} \boldsymbol{\Omega}-\frac{\nabla p \times \nabla \rho}{\rho^{2}} .
$$

that resembles very closely equation (9), provided we neglect the dissipation terms and we rescale the magnetic field by a factor $m_{i} / e(1+Z)$. Thus when vorticity is generated at cosmic shocks, magnetic field is also generated with strength $B=\Omega(1+Z) m_{i} / e$. From dimensional analysis we infer a vorticity of order the free fall rate, $t_{f f} \sim \sqrt{G \rho}$. Upon rescaling

$$
B \approx \frac{e(1+Z)}{m_{i}} \sqrt{G \rho} \approx 10^{-21}\left(\frac{n_{\mathrm{IGM}}}{10^{-4} \mathrm{~cm}^{-3}}\right)^{-\frac{1}{2}} \mathrm{G},
$$

with $n_{\mathrm{IGM}}$ the pareticle density in the intergalactic medium. This crude estimate is consistent with results from numerical simulations of structure formation [41].

The Biermann battery mechanism as a source of magnetic fields in the intergalactic medium was first proposed in the context of ionization fronts produced during the epoch of cosmic reionization [53]. Compared to shocks, this process produces larger and smaller seeds in high and low density regions respectively. In particular, a numerical model measures a volume averaged seed field of strength a few $\times 10^{-22} \mathrm{G}$ and a mass averaged seed of several $\times 10^{-19}$ $\mathrm{G}$, with peak values about an order of magnitude higher than that in the most dense regions [54].

\subsection{Resistive generation by cosmic-ray driven return currents}

Magnetic field generation can also occur in the presence of energetic particle currents. In the context of the intergalactic medium, large-scale magnetic fields can be generated due to a resistive instability excited by the return currents generated when high energy protons (heretofore CR for cosmic-ray) escape from the first generation of galaxies [47]. In this scenario the CRs are accelerated at supernova blast waves [55, 56, 57, 58] marking the death of the massive $\mathrm{OB}$ stars which produced the high energy photons responsible for the re-ionization of the intergalactic medium.

In the first galaxies, the magnetic fields were presumably much weaker than today ${ }^{3}$, such that the CRs, having a long mean free path, would naturally escape the parent galaxy in large quantities [59]. The resulting energetic particle flux propagating outwards into the IGM would be likely dominated by protons, electrons being typically fewer in number, as implied by modern day measurements of CRs arriving at earth, but also on account of their susceptibility to more severe energy losses [59]. Thus, the radial flux will carry a positive current $\mathbf{J}_{c}$ permeating the surrounding IGM. However, to maintain quasi-neutrality, a return current $\mathbf{J}_{r}$ must be drawn in the background plasma. Since the return current is carried by thermal electrons, which in the low temperature IGM plasma, will be subject to Coulomb

\footnotetext{
${ }^{3}$ or we wouldn't be having this discussion
} 
collisions, an electric field must be established in the background plasma, in order for the electrons to overcome the frictional drag of the heavy background ions. Returning to the generalised Ohm's law, equation (6), we see that the appropriate field is given by $\mathbf{E}=\eta \mathbf{J}_{r}$. This field opposes the propagation of the CR but is too weak to prevent their escape. In order to satisfy Ampére's law: $\mu_{0}\left(\mathbf{J}_{c}+\mathbf{J}_{r}\right)=\nabla \times \mathbf{B} \approx 0$, i.e. $\mathbf{J}_{r} \approx-\mathbf{J}_{c}$. The plasma resistivity in an unmagnetized medium is determined by its collisional rate which only depends on temperature, and can be written as, $\eta(T)=\eta_{0} T^{3 / 2}$ [49], where $\eta_{0}$ is a constant. Owing to the formation of structure in the universe, driven by gravitational instability, considerable density and temperature inhomogeneities existed in the IGM long before the onset of re-ionization. Thus the electric field is also inhomogeneous and in particular

$$
\nabla \times \mathbf{E}=\nabla \times \eta \mathbf{J}_{r} \approx-\frac{3}{2} \eta \mathbf{J}_{c} \times \frac{\nabla T}{T}
$$

where we have neglected the term $\propto \nabla \times \mathbf{J}_{c}$ (to lowest order, one would expect the CR current to be spherically symmetric) and have used Spitzer's expression for the resistivity. The important point is that, since the current is unrelated to the IGM inhomogeneities, the curl of the electric field is non vanishing. The rotational component of the electric field sustains Faraday's induction and generates magnetic field. The process is most efficient when the IGM temperature is low, which occurs before cosmic reionization. The analysis of Ref. [47] used the observed UV luminosity function of high redshift galaxies to estimate the production rate of CR in those galaxies and the ensuing return currents in the IGM, $\mathbf{J}_{r}$. They also used simulations of structure formation to estimate the temperature gradient scale-length, $T / \nabla T$, entering the curl of the electric field [48]. They found that magnetic field is robustly generated throughout intergalactic space at rate of $10^{-17}-10^{-16}$ Gauss/Gyr, until the temperature of the intergalactic medium is raised to $10^{4} \mathrm{~K}$ by cosmic re-ionization when the age of the universe is roughly $t=1 \mathrm{Gyr}$.

\subsection{Collisionless plasmas and Weibel instability}

In many astrophysical environments the interstellar and intergalactic medium is swept with supersonic flows, which drives hydrodynamic instabilities and shocks. While on the macroscopic scale these shocks behave like an ordinary shock, at the microscopic level their structure is very different from terrestrial ones. As an example we consider the outer shock seen in the Chandra X-ray observations of the supernova remnant SN 1006 [60] (see Fig. 1). Since the inferred post-shock density and temperature are $n_{i} \sim 1 \mathrm{~cm}^{-3}$ and $T_{i} \sim 15 \mathrm{keV}$, respectively, the Coulomb mean free path is thus $\sim 13 \mathrm{pc}$. This length is of the order of the diameter of the remnant $(\sim 10 \mathrm{pc})$ and much less than the observed shock thickness $\sim 0.04 \mathrm{pc}$ [61]. On Earth, the width of any shock is of the order of the collisional mean free path, but, in the case of SN 1006 it is evident that Coulomb collisions cannot be responsible for the observed width. Instead, nonlocal, non-binary, "anomalous" collisions between particles must be responsible for stopping the incoming flow and cause irreversible dissipation and heating in the downstream plasma. These are believed to be longrange collective interactions between particles and magnetic fields [62], and those mediate the formation of a so-called collision-less shock. Compelling evidence of the existence of such shocks in laboratory laser-based experiments has recently been provided [63].

Collision-less shocks thus require and, as direct consequence are sources of, electro-magnetic fields. For some conditions, like SN 1006, a magnetic field is already present and magnetised shocks can be produced [64, e.g.]. But we can not always expect a dynamically important magnetic field to be present. This is certainly the case of shocks arising during structure formation, when the magnetic field was still tiny. Shock formation is still possible, and this arises because, the initial ion distribution function of the interpenetrating flow is highly anisotropic: that is, consisting of two streams of counter-propagating ions. This situation is susceptible to a number of kinetic instabilities. One possibility is the formation of strongly fluctuating electric fields via the Buneman instability. The characteristic length of this instability is $[62,61] \ell_{E S} \sim K \lambda_{D}$, where $\lambda_{D}$ the Debye length, and the numerical factor, $K \gtrsim 1$, accounts for the number of growth times required for the instability to develop. Essentially, this instability is determined by Langmuir wave turbulence and it requires for the flow to have travelled several ion plasma wave oscillations. While this may be the mechanism for the formation of a weak shock (with Mach number $\lesssim 3.1$ ), it is generally believed that in the case of stronger shocks a more robust source of shock formation is the Weibel instability [42, 43]. What happens here is that in presence of counter streaming ions, a non-zero perturbation of the magnetic field perpendicular to the flow causes particles moving in one direction to concentrate at one point while the ones moving in the other direction to diverge. This creates a current that reinforces the initial perturbation and the magnetic field can thus rapidly grow. It 


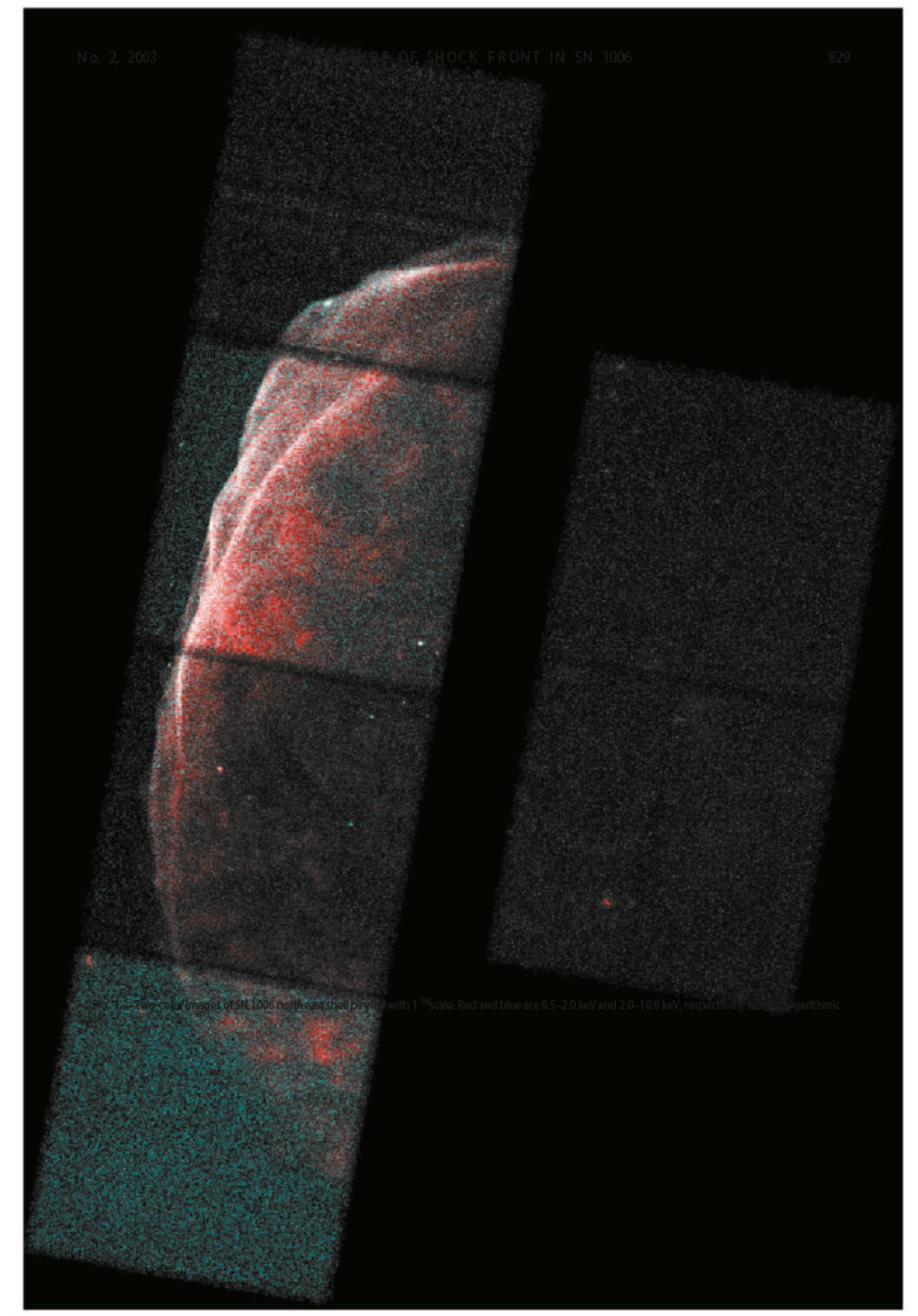

Figure 1: Composite image of SN 1006 in the $0.5-2.0 \mathrm{keV}$ (red) and 2.0-10.0 keV (blue) energy bands. Adapted from Ref. [60]. 
has been recently proposed $[44,45,64]$ that generation of magnetic fields can occur at cosmic shocks via the Weibel instability [42, 43]. Analytic estimates indicate that, for large Mach numbers, $M \gg 3$, the shock width is determined by the ion skin depth, i.e.,

$$
\ell_{E M} \sim K \frac{c}{\omega_{p i}} \approx 3 K \times 10^{-9}\left(\frac{n_{\mathrm{IGM}}}{10^{-4} \mathrm{~cm}^{-3}}\right)^{-1 / 2} \mathrm{pc},
$$

were $\omega_{p i}$ is the ion plasma frequency. The numerical coefficient $K \sim 10-100$ is determined by particle-in-cell (PIC) simulations $[45,64]$. On this scale, the magnetic field has reached saturation and it is near equipartition with the incoming ram pressure of the flow. This gives,

$$
B_{E M} \sim 10^{-8}\left(\frac{\eta_{B}}{10^{-3}}\right)^{1 / 2}\left(\frac{v_{s}}{10^{7} \mathrm{~cm} / \mathrm{s}}\right)\left(\frac{n_{\mathrm{IGM}}}{10^{-4} \mathrm{~cm}^{-3}}\right)^{1 / 2} \mathrm{G},
$$

where $v_{s}$ is the shock speed, and $\eta_{B}$ is an efficiency factor that is determined through numerical simulation [45, 46, 64]. Hence, while large magnetic fields can be produced by the Weibel instability, their scale is determined by the electromagnetic ion skin depth, $\ell_{E M}$, which is tiny on intergalactic scales, and thus their value is relevant only within the shock transition layer, while dissipating shortly afterwards.

\section{Similarity relations}

The discussion here closely follows that of Ref. [11], but with some simplifications in order to keep the treatment as straightforward as possible. The basic premise is that two systems, although vastly different in scale, can be accurately described in a fluid framework. For this to be true, a fluid description must be applicable in both the laboratory system and the astrophysical system. A number of assumptions must be made in this regard. Firstly, we must limit ourselves to low-frequency phenomena, occurring at frequencies well below the ion-plasma frequency. We also require some level of collisionality, such that the particle distributions can be treated as Maxwellian. While this is generally true in the laboratory investigations we discuss in this review, where binary Coulomb collisions make a major contribution, it is not necessarily true in astrophysical systems. However, as stated previously, where Coulomb collisions are insufficient to maintain near Maxwellian distributions, collisions off small-scale electromagnetic fluctuations is understood to provide a similar effect. In any case, assuming that two vastly disparate systems can indeed both be described within a fluid framework, there is still no guarantee that a laboratory fluid should behave in the same manner as an astrophysical fluid. The two systems will exhibit the same dynamics only under some specific conditions. In order to show how a similarity relation can be constructed, here we consider a special case of magneto-hydrodynamics (MHD) with negligible heat conduction, radiation diffusion or quantum effects. Inclusion of these effects is straightforward, but at the expense of a more cumbersome mathematical treatment $[65,66,67,68,69,2,70,71,11]$. We will consider further terms later on in this review. We assume the plasma is described by a single fluid, but with appropriate transport coefficients that are derived from kinetic theory [72].

We start by writing down the full set of MHD equations in the limits discussed above. The equation for the conservation of mass reads as

$$
\frac{\partial \rho}{\partial t}+\nabla \cdot \rho \mathbf{u}=0
$$

where $\rho$ is the mass density, $t$ the time and $\mathbf{u}$ the fluid velocity. The equation for conservation of momentum is

$$
\rho\left(\frac{\partial \mathbf{u}}{\partial t}+\mathbf{u} \cdot \nabla \mathbf{u}\right)=-\nabla p+\nabla \cdot \sigma_{v}+\mathbf{F}_{E M},
$$

where $p$ is the fluid pressure, $\sigma_{v}$ the stress tensor, and $\mathbf{F}_{E M}$ electromagnetic volume forces. In the case of an incompressible fluid, we have $\sigma_{v}=\rho v\left[\nabla \mathbf{u}+(\nabla \mathbf{u})^{T}\right]$, with $v$ the viscosity. The average Lorentz force on a given fluid element is given by $\mathbf{F}_{E M}=\rho_{C} \mathbf{E}+\mathbf{J} \times \mathbf{B}$, where $\rho_{C}$ is the charge density, $\mathbf{J}$ the current density, $\mathbf{E}$ the electric field, and $\mathbf{B}$ is the magnetic field.

In the absence of external heat sources, the equation for conservation of energy is

$$
\frac{\partial}{\partial t}\left(\rho \epsilon+\frac{\rho u^{2}}{2}\right)+\nabla \cdot\left[\rho \mathbf{u}\left(\epsilon+\frac{u^{2}}{2}\right)+p \mathbf{u}\right]=\nabla \cdot\left(\sigma_{v} \cdot \mathbf{u}\right)-\mathbf{J} \cdot \mathbf{E}
$$


where $\epsilon$ is the specific internal energy. Note that we have ignored a number of additional effects here, such as thermal diffusivity carried by either electrons or radiation.

Finally, our equations are completed with the equation for the evolution of the magnetic field, which, for now we can take to be the same as equation (4):

$$
\frac{\partial \mathbf{B}}{\partial t}=\nabla \times(\mathbf{u} \times \mathbf{B})+\frac{\eta}{\mu_{0}} \nabla^{2} \mathbf{B}
$$

This completes the set MHD equations that, in principle, describe the plasma evolution equally well in the laboratory as well as in a suitable astrophysical system.

In order to highlight relations between the MHD equations in two systems, we now rescale the variables in the hydrodynamic equations by a corresponding characteristic value. This allows us to rewrite the MHD equations in an invariant form, and all the details associated with the physical dimensions of the system are contained in characteristic dimensionless numbers. We write the velocity, position, mass density, current density, and electric field as

$$
\mathbf{u} \rightarrow u_{0} \mathbf{u}^{*}, \quad \mathbf{r} \rightarrow \ell_{0} \mathbf{r}^{*}, \quad \rho \rightarrow \rho_{0} \rho^{*}, \quad \mathbf{J} \rightarrow J_{0} \mathbf{J}^{*}, \quad \mathbf{E} \rightarrow E_{0} \mathbf{E}^{*}
$$

where $u_{0}, \ell_{0}, \rho_{0}, J_{0}$, and $E_{0}$ are the characteristic velocity, length, density, current density and electric field of the system, respectively. From now on we will use the convention that starred quantities (i.e. $\mathbf{u}^{*}$ ) are dimensionless, while quantities with subscript 0 (i.e. $u_{0}$ ) correspond to a characteristic value for that variable. We can also set

$$
t \rightarrow t_{0} t^{*}, \quad p \rightarrow p_{0} p^{*}, \quad \mathbf{B} \rightarrow B_{0} \mathbf{B}^{*}, \quad \epsilon \rightarrow \epsilon_{0} \epsilon^{*}, \quad \rho_{C} \rightarrow \rho_{C_{0}} \rho_{C}^{*} .
$$

However, the choice of the values for $t_{0}, p_{0}, B_{0}, \epsilon_{0}$, and $\rho_{C_{0}}$ is not arbitrary, but determined by the requirement that the ideal MHD equations (i.e. , without viscous and resistive dissipation) should remain scale invariant. It is thus possible to show [11] that $t_{0} \equiv \ell_{0} / u_{0}, p_{0} \equiv \rho_{0} u_{0}^{2}, \epsilon_{0} \equiv u_{0}^{2}$, and $B_{0} \equiv u_{0} \sqrt{\mu_{0} \rho_{0}}$. In addition, from Ampére's law and Ohm's law, we have $E_{0}=u_{0} B_{0}$ and $J_{0}=B_{0} / \ell_{0} \mu_{0}$ We see that the reference magnetic field has a value such that the fluid velocity and the Alfvén velocity [73] are the same. Similarly, charge conservation implies $\rho_{C_{0}}=J_{0} / u_{0}$. These assumptions also imply

$$
\frac{\partial}{\partial t} \rightarrow \frac{u_{0}}{\ell_{0}} \frac{\partial}{\partial t^{*}}, \quad \nabla \rightarrow \frac{\nabla^{*}}{\ell_{0}}
$$

Using the relations above, the MHD equations become [11]:

$$
\begin{gathered}
\frac{\partial \rho^{*}}{\partial t^{*}}+\nabla^{*} \cdot \rho^{*} \mathbf{u}^{*}=0 \\
\frac{\partial \mathbf{B}^{*}}{\partial t^{*}}=\nabla^{*} \times\left(\mathbf{u}^{*} \times \mathbf{B}^{*}\right)+\frac{1}{R m} \nabla^{* 2} \mathbf{B}^{*}, \\
\rho^{*}\left(\frac{\partial \mathbf{u}^{*}}{\partial t^{*}}+\mathbf{u}^{*} \cdot \nabla^{*} \mathbf{u}^{*}\right)=-\nabla^{*} p^{*}+\frac{1}{R e} \nabla^{*} \cdot\left[\nabla^{*} \mathbf{u}^{*}+\left(\nabla^{*} \mathbf{u}^{*}\right)^{T}\right]+\rho_{C}^{*} \mathbf{E}^{*}+\mathbf{J}^{*} \times \mathbf{B}^{*}, \\
\frac{\partial}{\partial t^{*}}\left(\rho^{*} \epsilon^{*}+\frac{\rho^{*} u^{* 2}}{2}\right)+\nabla^{*} \cdot\left[\rho^{*} \mathbf{u}^{*}\left(\epsilon^{*}+\frac{u^{2}}{2}\right)+p^{*} \mathbf{u}^{*}\right]=\frac{1}{R e} \nabla^{*} \cdot\left[\nabla^{*} \mathbf{u}^{*}+\left(\nabla^{*} \mathbf{u}^{*}\right)^{T}\right] \cdot \mathbf{u}^{*}-\mathbf{J}^{*} \cdot \mathbf{E}^{*} .
\end{gathered}
$$

In these equations, we recognize familiar dimensionless numbers. The fluid Reynolds number, $R e=u_{0} \ell_{0} / v$, represents the ratio of inertial to viscous effects. Similarly, the magnetic Reynolds number $R m=\mu_{0} u_{0} \ell_{0} / \eta$, gives the ratio of inertial to resistive processes. Similarity between the laboratory and astrophysical object is achieved if these dimensionless numbers are the same in both systems. However, exact similarity is rarely achievable, the Reynold's numbers in astrophysical systems, taken at face value, typically out of reach for laboratory plasma conditions. The alternative viewpoint is that if $R e$ and $R m$ are sufficiently large in both systems, each can be treated as infinite Reynolds number plasmas, and again, similarity is achieved. One should however always take care when making such an approximation. That either system behaves as an infinite Reynolds number fluid is not certain, and of course, in turbulent systems, strong non-local, multi scale coupling is always a possibility. Nevertheless, provided due care is taken, laboratory experiments may be representative of their astrophysical counterparts as long as ideal conditions are 
achieved at some representative scale. To quantify this statement in a more formal way, we take $\ell_{0}^{(1)}, u_{0}^{(1)}, \rho_{0}^{(1)}$ and $J_{0}^{(1)}$ as the characteristic values for the parameters in a given laboratory experiment. The corresponding values in the astrophysical system are then simply found by the following linear scalings:

$$
\ell_{0}^{(2)}=g_{a} \ell_{0}^{(1)}, \quad u_{0}^{(2)}=g_{b} u_{0}^{(1)}, \quad \rho_{0}^{(2)}=g_{c} \rho_{0}^{(1)}, \quad J_{0}^{(2)}=g_{d} J_{0}^{(1)},
$$

where $g_{a, b, c, d}$ are scaling constants. From this set of parameters, we can scale all the other characteristic quantities as

$$
t_{0}^{(2)}=\frac{g_{a}}{g_{b}} t_{0}^{(1)}, \quad p_{0}^{(2)}=g_{c} g_{b}^{2} p_{0}^{(1)}, \quad B_{0}^{(2)}=g_{b} \sqrt{g_{c}} B_{0}^{(1)}, \quad \epsilon_{0}^{(2)}=g_{b}^{2} \epsilon_{0}^{(1)}, \quad \rho_{C_{0}}^{(2)}=\frac{g_{d}}{g_{b}} \rho_{C_{0}}^{(1)} .
$$

Thus, we have simple relationships that allow us to map the parameters in one system onto the other.

Finally following the same approach as above, the dimensionless form of the induction equation with the Biermann battery source term (Eq. 9) is

$$
\frac{\partial \mathbf{B}^{*}}{\partial t^{*}}=\nabla^{*} \times\left(\mathbf{u}^{*} \times \mathbf{B}^{*}\right)+\frac{1}{R m} \nabla^{* 2} \mathbf{B}^{*}+\frac{1}{B i} \frac{\nabla^{*} p^{*} \times \nabla^{*} \rho^{*}}{\rho^{* 2}},
$$

where we define Biermann number,

$$
B i=\frac{e \sqrt{\mu_{0} \rho_{0}} \ell_{0}(1+Z)}{m_{i}}=(1+Z) \frac{e B_{0}}{m_{i}} t_{0} .
$$

Clearly, from the last expression, we see that this term is negligible in a magnetised plasma, $B i \sim \omega_{g} t_{0} \gg 1$ $\left(\omega_{g}=e B_{0} / m_{i}\right)$, but if the field is initially very weak, the Biermann term can completely dominate the magnetic field evolution.

\section{Numerical MHD and analogue experiments}

The above equations of MHD contain within them, a countless number of physical processes with solutions describing the motion of simple plasma waves, shocks, magnetic reconnection, turbulence, current sheets, dynamos, etc. However, even in the ideal limit $(\eta, v \rightarrow 0)$, the equations are strongly non-linear, and although some exact solutions can be found, an approach to solving these equations analytically, at least in any systematic sense, is currently unknown. Numerical approaches are thus desirable, although construction of accurate schemes that can handle any and all possible situations is challenging. This is perhaps not surprising, as any numerical approach relies on the ability to approximate a system with an infinite number of degrees of freedom using a discretised (finite) representation of the dependent variables. From this perspective, the advantage of laboratory experiments is self-evident. In addition, at large Reynolds numbers, Re $\gg 1$, which is the regime most applicable in both astrophysical and the laboratory plasmas we discuss here, experiments have shown that the flow is unstable to even minute fluctuations. Turbulence is the natural outcome, which involves multi-scale coupling from some outer scale to the dissipation scale. When we take these points into consideration, the inherent difficulty in constructing a generalised numerical approach is clear. Luckily, in most circumstances, it is possible to identify, and even quantify the limitation of a given approach, and provided due care is taken, MHD simulations offer a powerful tool to study complex plasma behaviour in the fluid limit. These days, numerical simulations are almost a necessity for the interpretation of experimental data, making the two fields, numerical MHD and experiment, complimentary.

The study of numerical methods for MHD is an active research field in its own right, and an impressive number of different techniques have been developed. Which approach is best suited to a given problem will depend on the physical process under investigation. Grid based codes, where the dependent parameters are discretised on a spatial grid, are certainly the most common (see Ref. [74] for a brief description of several different grid based codes, and a comparison of the results for a given problem with matching initial conditions). Shock capturing schemes are easily implemented in this framework, and are particularly attractive for most of the laboratory investigations discussed in this review, since shocks are often produced in these experiments. These schemes are also the most commonly used in astrophysical simulations, although MHD extensions of smoothed particle hydrodynamics (SPH) codes are also frequently used in cosmological simulations. While these codes easily generate shock waves, that trivially satisfy 
the Rankine-Hugoniot relations, their ability to handle multi-scale coupling is less clear, and the role of numerical viscosity and resistivity is an ever-present issue.

The maximum Reynolds number that can be achieved in numerical simulations demands some further discussion, and distinguishing between physical and numerical effects is an important, but rarely discussed issue. Even in the ideal MHD limit, numerical viscosity is unavoidable, since any numerical errors will generally act like a viscosity or hyper-viscosity term. Generally speaking, for grid based codes, viscosity is favourable, as it damps unwanted grid scale fluctuations. It is possible to place somewhat crude limits on the effective numerical Reynolds number in such simulations. Since flow on the grid scale is inevitably laminar, one should expect to have $\operatorname{Re}=\bar{u} \Delta x / v \sim 1$ at the grid scale ( $\Delta x$ is the grid spacing, and $\bar{u}$ the characteristic velocity at that scale), rescaling up to the simulation domain $L=N \Delta x$, where $N$ is the number of grid cells in a given dimension, we expect $N<\operatorname{Re}<N^{2}$, the upper limit unlikely to be realised in practise. A similar argument holds for the magnetic Reynolds number, and in general, it is thought that such simulations, in the absence of applied resistivity/viscosity, are limited to Prandtl numbers of order unity. It is, in principle, a straightforward matter to include resistivity and viscosity in these simulations, however this introduces additional problems, since it is important to resolve the resistive scale [75], which shrinks to minute scales with decreasing viscosity, $\ell \propto v^{3 / 4}$. One must also ensure that the physical viscosity and resistivity dominate over their numerical counterparts, which is not always trivial to quantify. Various sophisticated methods have been developed to minimise the computational effort in reproducing large Reynolds numbers, while still resolving/capturing the scales of interest. Adaptive Mesh Refinement (AMR) is now included in many MHD codes [76, 77, 78, 79]. The use of high order schemes with hyper-viscosity/resistivity have also been used successfully in turbulence simulations [23]. Moreover, conceptually trivial processes such as the Biermann battery cannot always be easily implemented in fluid codes [80]. Spectral codes are perhaps the best suited methods to study turbulence [81, 75], but are of limited use when it comes to modelling laboratory shock experiments.

Ultimately, any numerical approach can only approximate the physical system, and relies heavily on the ingenuity of the programmer. Experiments on the other hand make no approximation on the microphysics (Ohm's law, scalar viscosity/resistivity). Current laser plasma experiments have succeeded in producing ionised fluid flows with Reynolds numbers $\operatorname{Re}>10^{4}$. While this still falls short of what is typically of interest in astrophysical scenarios, it is still better than what can be realitistically achieved using computational resources available today. Producing Magnetic Reynolds numbers in current experiments are unfortunately more modest, typically falling in the regime $\mathrm{Rm} \gtrsim 1$. However, the fields are typically sufficiently weak that a hydrodynamics scaling is unaffected, while MHD scaling can be achieved within a certain range [82, 11]. With academic access to large facilities such as OMEGA [83], NIF [84] and LMJ [85], higher magnetic Reynolds numbers can be achieved, but balancing of other effects becomes increasingly important. We will discuss this later.

\section{Laser facilities for laboratory astrophysics}

As stated above, numerical simulations offer a powerful aid for investigations of fundamental plasma physics, but clearly comes into its own in its ability to model astrophysical systems. While astrophysical systems can not be reproduced in the laboratory (a fact that is important to always bear in mind), it is possible to isolate and study specific processes of astrophysical relevance. This represents a completely different approach, but is in many regards complimentary to numerical and theoretical studies, since, once identified and understood, it removes any ambiguity associated with numerical effects, etc. The resulting physics, subject to the above discussed constraints, can subsequently be scaled to their astrophysical counterparts, providing tangible numbers that can quantify the relevant parameters. If we want to reproduce in laboratory experiments conditions representative of the intergalactic medium, we need to access facilities where strong shocks can be generated in an initially unmagnetized plasma. While high power lasers are not the only type of facilities available for laboratory astrophysics experiments (see e.g, Ref. [3]), they meet all the requirements needed to investigate the evolution of magnetic fields from tiny seeds to larger one via amplification driven by shocks and turbulence.

The experiments discussed in this review have been carried out at different laser facilities across the globe. To illustrate the way these lasers work, we consider as an example, what is currently the largest laser system in world: the National Ignition Facility (NIF) laser, shown in Figure 2 [86]. As the name suggests, the main motivation behind the development of this large laser facility is the challenge of achieving inertial confinement fusion [87], i.e., using several laser beams to implode a deuterium-tritium capsule and initiate self-sustained fusion reactions [88]. These lasers start 


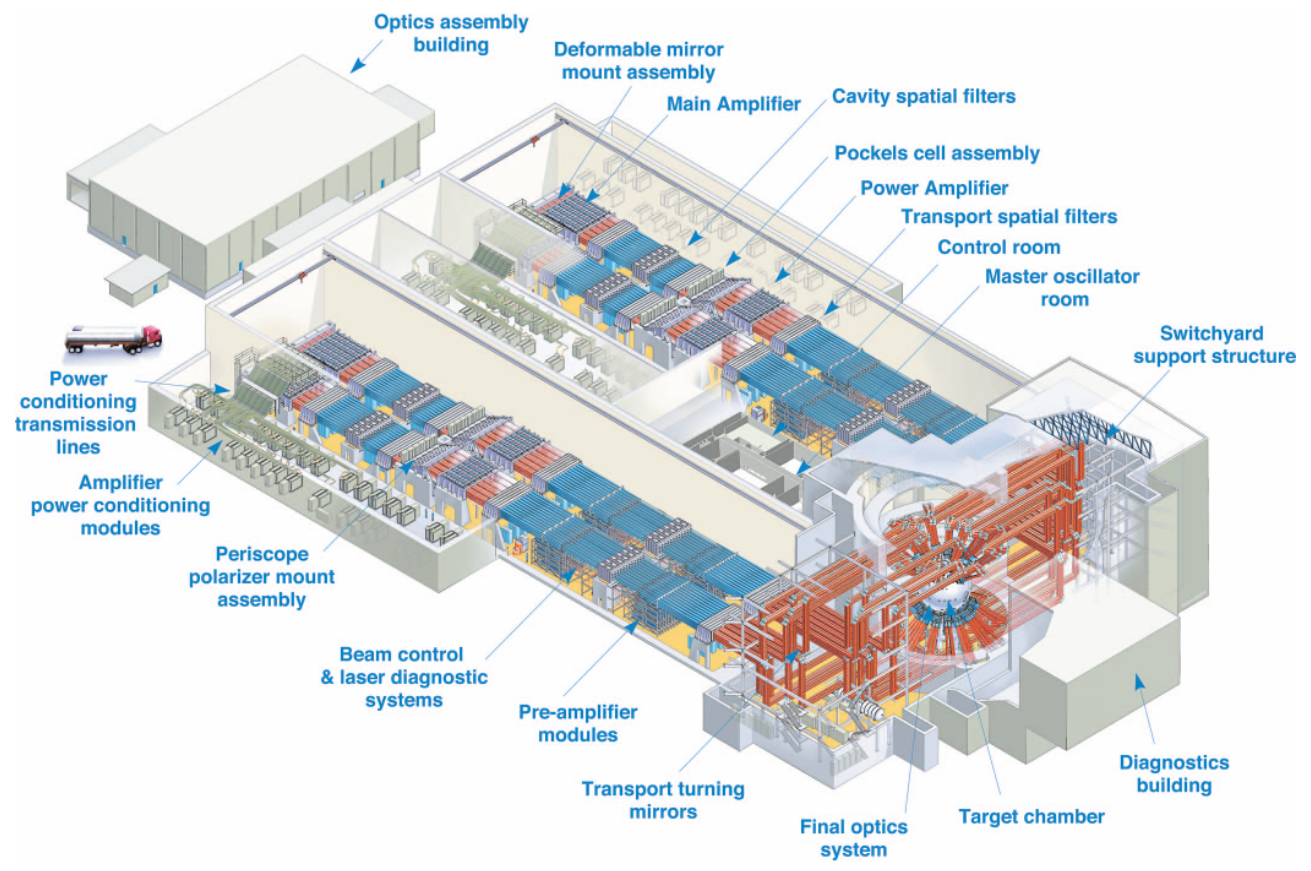

Figure 2: Schematic layout of the NIF laser and its main components. Adapted from Ref. [86].

very similar to any ordinary lasers, with a low energy beam, but with very high quality spatial and temporal profile. Then as the beam propagates along the laser chain, it is progressively expanded and amplified in flashlamp-pumped Nd-doped glass. Even so, the energy per unit area on the glass reaches values that are close to the damage threshold and this significantly reduces the shot rate to only a few per day. All of these lasers operate at the fundamental frequency of $1054 \mathrm{~nm}$, but prior to delivery they are often converted to double or triple the operating frequency with KDP crystals. The NIF laser has 192 beams, and each one can be independently pointed, focussed and timed. The laser spot on each beamline can be further controlled using a phase plate, diffractive plate that allows a pre-determined spatial distribution of the laser energy over a spot diameter ranging from a few hundreds of microns to a few millimeters. Since its commissioning a few years ago, experiments with the NIF laser have been largely dedicated to ignition campaign experiments (see Ref. [89] for the latest results), and only very recently it has opened for discovery science experiments. For this reason, the laboratory experiment discussed here have all employed smaller laser systems, but in the next years further progress on NIF is expected to occur. We will discuss these points further at the end of this review.

\section{Scaled laboratory experiments}

Here we discuss a laboratory experiment designed to verify the Biermann battery mechanism at shocks [90] and its relation to protogalactic magnetic field generation. The experiment was conducted at the LULI 2000 laser facility [91]. The details of the experimental setup are given in Fig. 3. Either one or two frequency-doubled (527 nm), 1.5 ns-long laser beams were focussed on the tip of a $500 \mu \mathrm{m}$ diameter carbon rod. At focus, each laser beam had a 400 $\mu \mathrm{m}$ flat-top distribution achieving a peak intensity of $2 \times 10^{14} \mathrm{~W} / \mathrm{cm}^{2}$, delivering $\sim 350 \mathrm{~J}$. The interaction chamber was filled with helium gas at pressure $p=0.8 \pm 0.3$ mbar and $p=1.6 \pm 0.3$ mbar. As energy is impulsively deposited, the sample is heated and initially undergoes a ballistic expansion until the shocked mass is roughly equal to the ejected mass. The shock transitions to a Sedov-Taylor blast wave [92, 93].

The experiment was designed to monitor the properties of the plasma and the shock with a number of different diagnostics. The shock wave evolution during times $t \lesssim 200 \mathrm{~ns}$ (where $t=0$ corresponds to the time of the laser pulse) was monitored using transverse interferometry and Schlieren/shadowgraphy [94] with an optical probe (with 


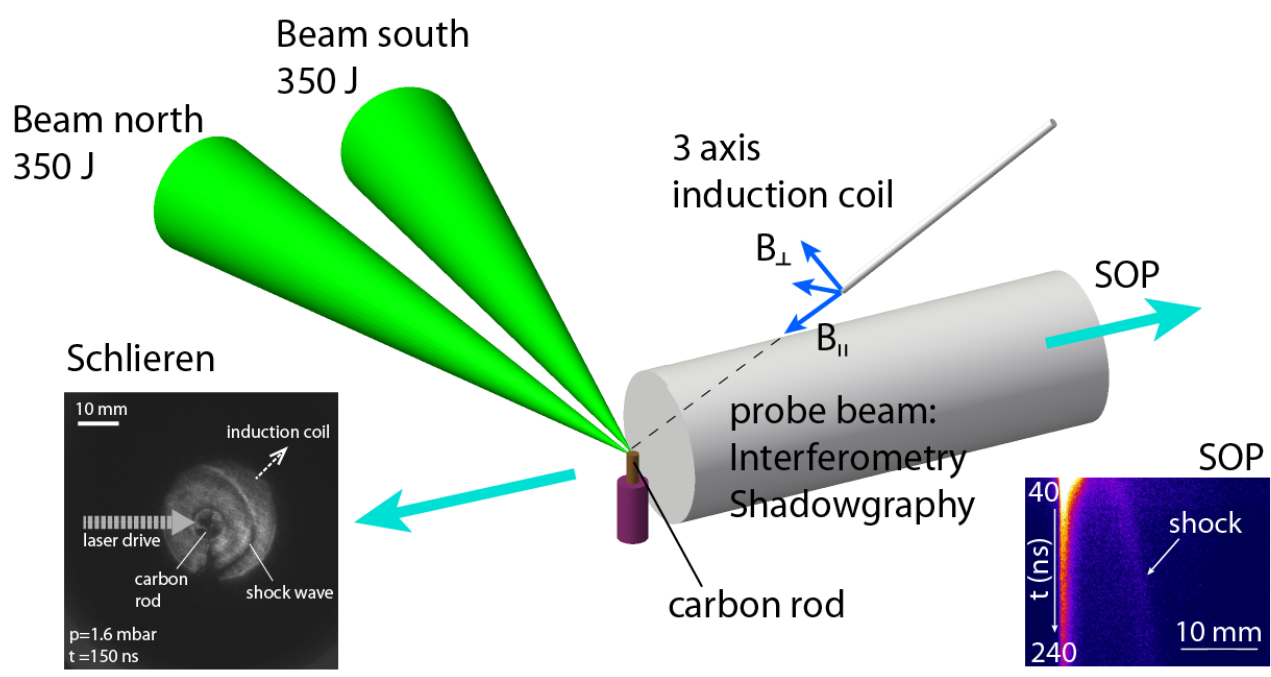

Figure 3: Experimental setup showing the laser beams and diagnostics configuration. The insets show an example of a Schlieren on the left, and an optical streak pyrometry (SOP) on the right. Adapted from Ref. [90].

$532 \mathrm{~nm}$ wavelength and $6 \mathrm{~ns}$ gate width). The interferometer was of Mach-Zehnder type with $\sim 25 \mathrm{~mm}$ field of view. Under the assumption of cylindrical symmetry (i.e., via an Abel inversion), the interferometry provides the electron density, and the Schlieren image shows when the refractive index of the plasma changes rapidly, thus tracking the position of the shock. Streaked self-emission optical pyrometry (SOP) in a narrow band at $450 \pm 30 \mathrm{~nm}$ (Fig. 3) was also used to track the evolution of the shock.

The experimentally measured shock position for $t \lesssim 200 \mathrm{~ns}$ was compared with a numerical simulation for the plasma evolution (Fig. 4). This was performed using HELIOS-CR [95], a hydrodynamic code which include laser deposition, radiation transport and tabulated equation of state for non ideal plasmas. The computation was carried in 1-dimension with spherical geometry. The laser energy deposition in the HELIOS-CR code was adjusted to have the simulated shock position match the experimental values. Fig. 4 shows the agreement between the calculated electron density profile for $t \lesssim 200 \mathrm{~ns}$ and the electron density profile from the optical interferometry. Based on this quantitative agreement the simulations were used to interpret the experimental results at later times, $t \sim 0.5-1.5 \mu \mathrm{s}$, when the shock arrives at the position of the magnetic field probes (see next section) but the plasma density is below the detection limit of the optical probe. Results from these simulations are shown in Fig. 5.

The scale invariance of the MHD equations can be exploited to draw an equivalence between the laboratory experiment and the collapsed intergalactic medium. For the conditions of this experiment we have $R e \approx 1,300$, $R m \approx 3$, and $B i \approx 10$ at a scale $\ell_{0} \sim 3 \mathrm{~cm}$ (set by the radius of the shock wave at the position of the magnetic field measurements). Also, since the Biermann induced currents remains small throughout the experiment, we can neglect both resistive and Hall diffusion compared to convective transport. Similar arguments can be made for thermal and radiation transport. Thus, on scales larger than $\ell_{0}$ the ideal MHD approximation is valid to describe the experiment [90]. In the case of collapsing protogalactic structures, the dimensionless numbers are all very large [6, 90], hence there is a direct similarity between the astrophysical and the laboratory systems under consideration.

\section{Magnetic field measurements}

Magnetic fields in the experiment are measured with induction coils [96], and they provide both the magnetic field components along the shock normal $\left(B_{\|}\right)$and perpendicular to it $\left(B_{\perp}\right)$ at $2.8 \mathrm{~cm}$ and $3.6 \mathrm{~cm}$ from the carbon rod. The probe design is based on the original work of Everson et al. [96] (see Fig. 6). The idea behind this diagnostics is relatively simple: a time varying magnetic field induces an electromotive force on a small loop and the measured 
(a)

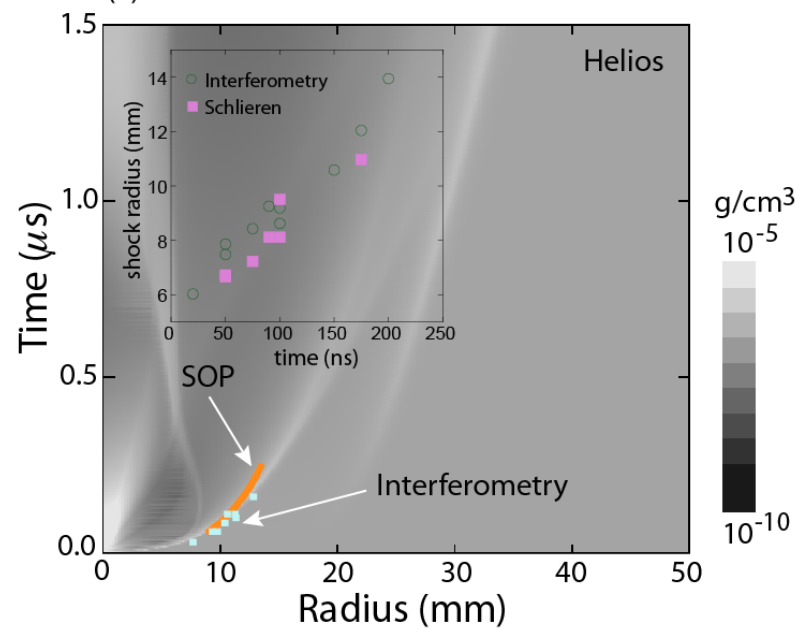

(b)

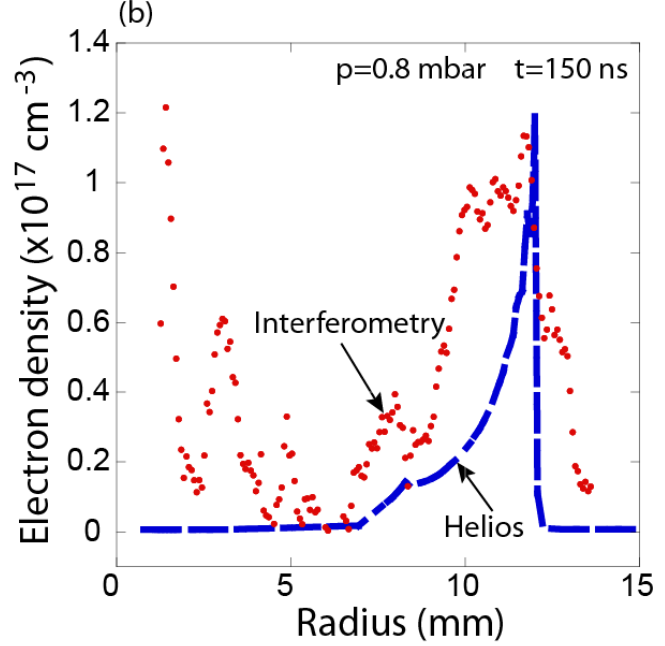

Figure 4: (a) HELIOS-CR simulations (done for $p=1$ mbar with one beam laser driver) and experimentally determined shock position with both interferometry and SOP. The inset shows an expanded view of the measured shock position. (b) Calculated electron density at $t=150 \mathrm{~ns} v s$ electron density values extracted from interferometric measurements via Abel inversion. Adapted from Ref. [90].

(a)

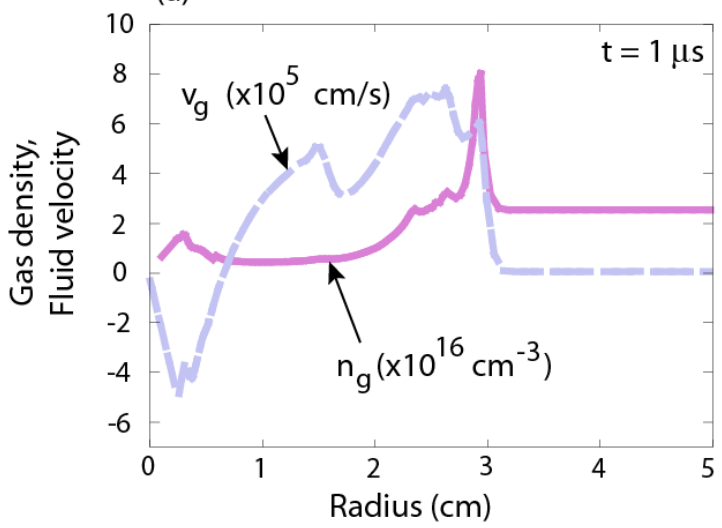

(b)

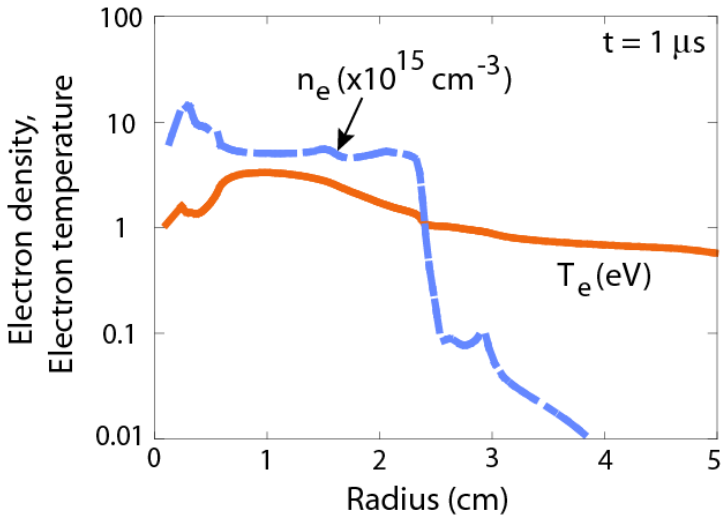

Figure 5: HELIOS-CR calculated profiles for the flow velocity $\left(v_{g}\right)$, gas density $\left(n_{g}\right)$, electron density $\left(n_{e}\right)$ and electron temperature $\left(T_{e}\right)$ at $t=1$ $\mu$ s. Adapted from Ref. [90]. 
(a)

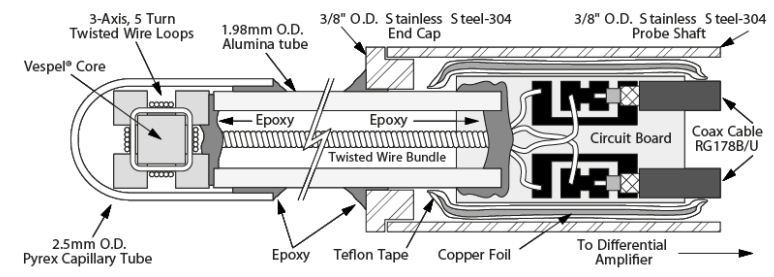

(b)

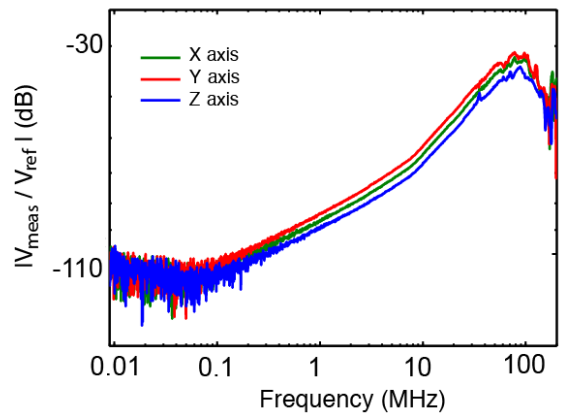

Figure 6: (a) Schematic of the induction coils sensor. From Ref. [96]. (b) Typical frequency response of the probe. From Ref. [82].

voltage $V_{\text {meas }}$ is related to the magnetic field by [96]:

$$
a N g \frac{d B(t)}{d t}=\left(1+\tau_{s} \frac{d}{d t}\right) V_{\text {meas }}(t)
$$

where $a$ is the cross sectional area of the loop, $N$ is the total number of windings, $g$ is the gain associated to operational amplifier used in the acquisition electronics, and $\tau_{s}$ accounts for the self-inductance and mutual-inductance in the induction probe sensor. Eq. (29) can be directly integrated in order to obtain $B(t)$ from the measured voltage waveform. Alternatively, it is possible to take the discrete Fourier transform of (29), solve for $B(\omega)$ and then apply the inverse discrete Fourier transform in order to retrieve the real-time dependence of the magnetic field. This latter method is often more efficient in the presence of noisy data and when there is a slowly varying background field that persists for times longer than measurement time period. The use of a windowing function to remove the offset at $\omega=0$ is thus needed.

The induction coils are calibrated in a known magnetic field generated by a set of Helmholtz coils. This allows to determine accurately the coefficients in (29). A vector network analyzer is used to drive the Helmholtz coils with a voltage that is frequency swept from $f=9 \mathrm{kHz}$ to $f=500 \mathrm{MHz}$. The measured signals in the induction coil probe $\left(V_{\text {meas }}\right)$ and in the reference Helmoltz coil $\left(V_{\text {ref }}\right)$ are simultaneously recorded. It can be shown that the relation between these two voltages is [96]

$$
\frac{V_{\text {meas }}(\omega)}{V_{\text {ref }}(\omega)}=a N g\left(\frac{16}{5^{3 / 2}}\right) \frac{\mu_{0}}{r R_{p}} \frac{\omega}{1+\left(\tau_{s} \omega\right)^{2}}\left[\omega\left(\tau_{s}-\tau\right)+i\left(\omega^{2} \tau_{s} \tau+1\right)\right]
$$

where $\omega=2 \pi f, R_{p}$ is the value of the resistor across which $V_{r e f}$ is measured, $r$ is the Helmholtz coil radius, and $\tau$ is the time delay due to the cable length difference between the probe and magnetic field generator circuit. Both $a$ and $\tau_{s}$ are determined from Eq. (30) by fitting the slopes of the imaginary and real components of $V_{\text {meas }}(\omega) / V_{\text {ref }}(\omega)$ for frequencies between 0.1 to $\sim 1 \mathrm{MHz}$ (see Fig. 6).

In the experiment of Fig. 3, each induction coil consists of 8 twisted pairs coils wound around the axis of a $\sim 3.1 \times 3.1 \mathrm{~mm}^{2}$ plastic core. The voltage from the twisted pair loops is then differentially amplified in order to remove any electric field components. The coils are protected from the surrounding plasma by a $1 \mathrm{~mm}$ thick glass tube. The position of the induction coil with respect to the carbon rod is shown in Fig. 3. From the calibration and the frequency response curve, the time resolution was shown to be better than $50 \mathrm{~ns}$.

Fig. 7 shows magnetic field traces from the induction probe. At $\sim 3 \mathrm{~cm}$ from the carbon rod, peak $B_{\perp}$ values occurring at $t \sim 1-2 \mu \mathrm{s}$ are in the range 10-30 G. The rise and gradual decay of $B_{\perp}$ is consistent with the shock front crossing the coil and the subsequent evolution of the shocked material. The position of the first peak in $B_{\perp}$ is in agreement with the shock arrival time estimates based on the HELIOS-CR simulations, consistent with the time lags between different locations of the probe coils. Finally, shots taken with no ambient gas show no peak in $B_{\perp}$ for $t \sim 0.5-2 \mu \mathrm{s}$, which further supports the inference that the magnetic field arrives with the shocked plasma. 
(a)

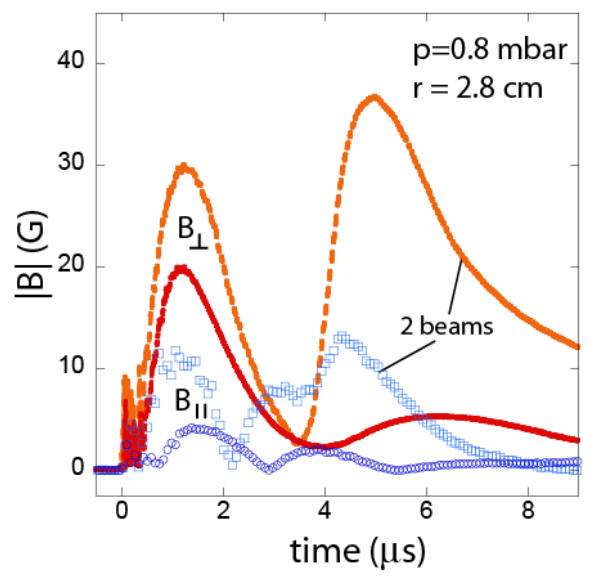

(b)

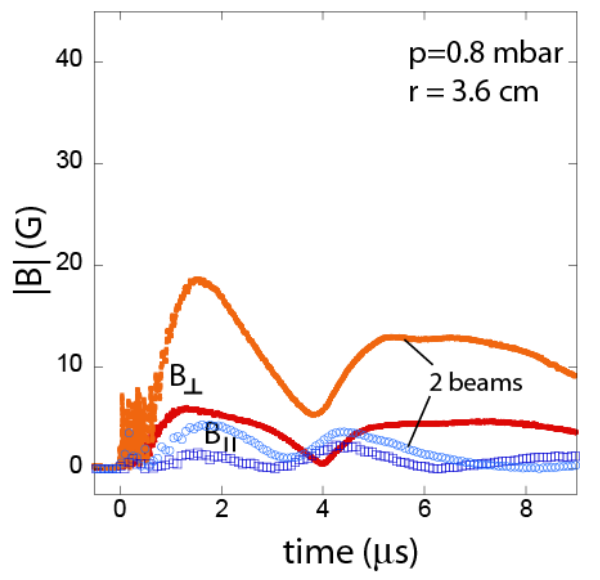

Figure 7: Magnetic field measurements. (a) $B_{\perp}$ (lines) and $B_{\|}$(symbols) traces taken at $p=0.8$ mbar. (b) Same as (b) but at $r=3.6 \mathrm{~cm}$. Adapted from Ref. [90].

\section{Laboratory Experiments of Biermann's Battery}

The experimental data shown in Fig. 7 suggests that a magnetic field could be produced at the shock. In order to assess the mechanism of magnetic field generation in these experiments it is necessary to consider a few different possibilities. It is well known for example that return currents driven by hot electrons can play an important role for field generation in laser produced plasmas [97] and in astrophysical contexts [98]. Given the laser intensity used in the experiment $\left(I=2 \times 10^{14} \mathrm{~W} / \mathrm{cm}^{2}\right)$ we expect the hot electrons to be distributed with temperature $T_{h o t} \sim 5$ $\mathrm{keV}$ [99], but they would reach the coil position at $t \lesssim 3 \mathrm{~ns}$, much earlier than the measured peak in $B_{\perp}$. It is also known that laser irradiation of solid foils produces strong magnetic fields $[50,100]$, with values $\sim 1 \mathrm{MG}$. This field develops near the critical density, $n_{c r} \sim 4 \times 10^{21} \mathrm{~cm}^{-3}$ for second harmonic illumination as in the experiments. The critical surface roughly expands at the sound speed $c_{s} \sim 2 \times 10^{7} \mathrm{~cm} / \mathrm{s}$, and the plasma maintains a temperature $\sim 1$ $\mathrm{keV}[2,101]$. Since the temperature is high near the laser spot, the magnetic Reynolds number is also large and the field is frozen-in the flow. It is thus advected and transported at the coil location. Assuming a Sedov expansion, in the case of perfect flux freezing, conservation of the magnetic flux implies $B_{\text {laser }} \sim 10^{6}\left(n_{e} v_{s}^{2} / n_{c r} c_{s}^{2}\right)^{2 / 3} \approx 0.2 \mathrm{G}$, where $n_{e}$ is the electron density at the coil position and $v_{s}$ the shock velocity there. This is likely un upper estimate, since the frozen-in conditions are, strictly speaking, only applicable in the initial phase of the expansion, very near to the laser focus. As the plasma cools, magnetic diffusion will start contributing, lowering even more the field values at the coil position. Thus, given the values of the measured magnetic fields, this contribution can also be ignored.

More likely here is the possibility of a magnetic field generated through vorticity by Biermann's battery [41]. We have shown earlier that the Biermann battery field is given by

$$
\left.\frac{\partial B}{\partial t}\right|_{B B} \approx-\frac{m_{i}}{e} \nabla \times\left(\frac{\nabla p}{\rho}\right)
$$

where we have taken $Z \sim 1$ (see Fig. 5). Neglecting electromagnetic and viscous forces, we have from the momentum equation $-\nabla p=\rho(\partial \mathbf{u} / \partial t+\mathbf{u} \cdot \nabla \mathbf{u})$, thus

$$
\left.\frac{\partial B}{\partial t}\right|_{B B} \approx \frac{m_{i}}{e} \frac{\partial \mathbf{\Omega}}{\partial t}
$$

where $\boldsymbol{\Omega}$ is the vorticity, previously introduced in the section 3.1 . Hence, $B=m_{i} \Omega / e$, and the magnetic field can be simply estimated by evaluating the vorticity in the flow. The justification for this last statement, follows from observation shown previously (see eqns 9 and 10) that the magnetic field and fluid vorticity satisfy virtually identical equations [102], with the implication that if both start from the same initial conditions, their subsequent evolution 

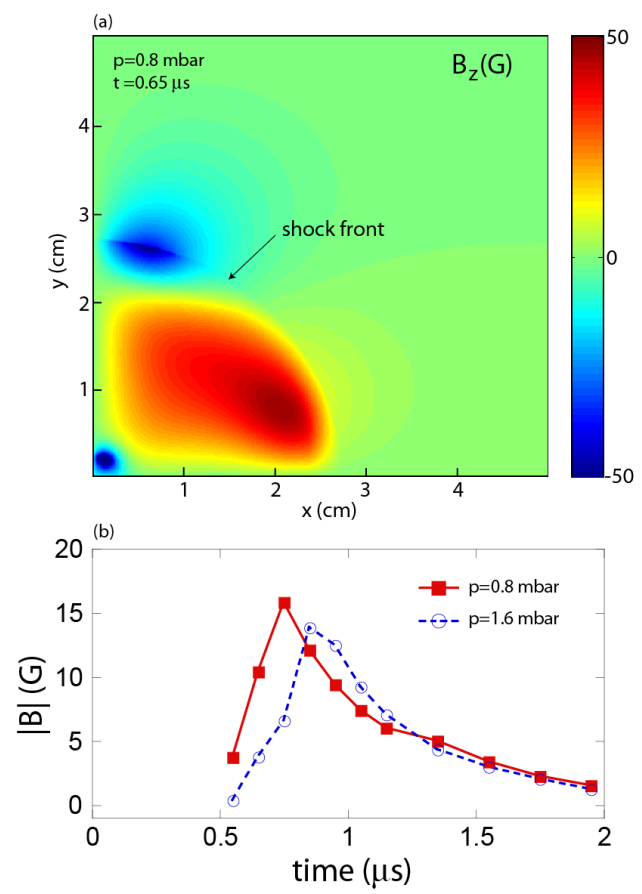

Figure 8: (a) 2-dimensional plot of the perpendicular component of the magnetic field obtained from MHD simulations. (b) Plot of the magnetic flux density at $2.7 \mathrm{~cm}$ from the center of the domain $(x=2.5 \mathrm{~cm}, y=1 \mathrm{~cm})$.

should match. In this picture, the vorticity can be evaluated analytically as [103, 104]

$$
\Omega \approx \frac{(\xi-1)^{2}}{\xi}\left|\frac{\partial \mathbf{v}_{s}}{\partial S}\right|,
$$

where $\xi$ is the shock compression ratio and $\partial \mathbf{v}_{s} / \partial S$ is the tangential gradient of the shock velocity. We approximate $\partial \mathrm{v}_{s} / \partial S \sim \kappa \mathrm{v}_{s} / r$, where $\kappa \sim 0.1-0.3$ is estimated from the observed shock asymmetry in Fig. 3. Using the inferred values from HELIOS-CR (see Fig. 5), with a density jump $\xi \sim 3$ at $r=3 \mathrm{~cm}$, we estimate $B \approx 10-30 \mathrm{G}$, in agreement with the observed values. In addition, the vorticity generated field is perpendicular to the shock normal, consistent with the experimental traces of Fig. 7 that show $B_{\perp} \gg B_{\|}$. We attribute shot-to-shot variations in the observed field values (up to $\sim 50 \%$ ) to the stochastic generation of vorticity and to variations in the radius of curvature of the shock front (as large as $\sim 20 \%$ at early times, as shown in Fig. 7).

To corroborate this interpretation, numerical simulations have been carried in 2-dimensions by solving the resistive MHD equations with a a baroclinic (i.e., Biermann battery) source term for the magnetic field. The ionization state is computed self-consistently using Saha equilibrium [68]. The results are shown in Fig. 8 with the MHD scheme implemented as described in Ref. [105]. Radiation transport, thermal conduction and laser coupling were not included in the MHD code. The initial conditions were determined by the results from HELIOS-CR simulations. At $t=150$ ns (i.e., the time when the shock has swept $\sim 1 \mathrm{~cm}$ from the initial carbon rod position), the system has uniform density and temperature, except for a central region $(\mathrm{r}<1 \mathrm{~cm})$ where the temperature is, $T(x, y)=T_{0}(1+\delta \cos \theta)$, with $T_{0}=50 \mathrm{eV}$ and $\delta=0.9$, and $\theta \in[0,2 \pi]$ the polar angle. Thus the scale coefficient $\kappa \sim 1 / \pi$. The numerical grid consists of $2000 \times 2000$ cells with resolution $\Delta x=\Delta y=0.005 \mathrm{~cm}$. The simulation results are shown in Fig. 8 . The asymmetric expansion due to the initial temperature anisotropy drives baroclinic generation of a magnetic field comparable in strength to what is measured in the lab. Note that because of finite magnetic diffusion, the plasma is magnetized ahead of the shock front.

Once that the magnetic field generation at shocks due to the Biermann battery mechanism has been established in the experiment, the next step consist in applying the similarity relations and scale this results to the intergalactic 


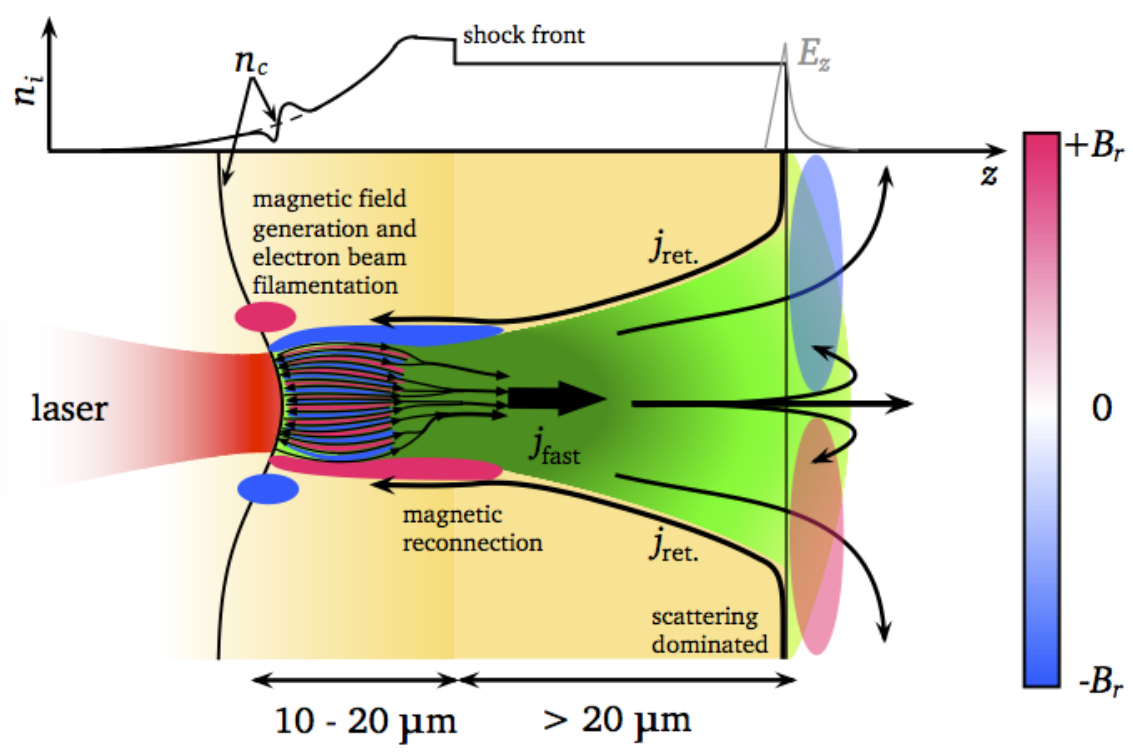

Figure 9: Schematic of a laser-produced fast-electron beam transport $\left(j_{\text {fast }}\right)$ into a solid target and consequent formation of a return current $\left(j_{\text {ret }}\right)$ and a global magnetic field. The interaction of the direct and return current leads to the formation of density and magnetic field filaments by the Weibel instability. At the rear side, the electrons form a sheath and build up an electrostatic field $E_{z}$, which can lead to re-circulation of the fast electrons and further enhancement of the process above. From Ref. [106].

medium. Following the discussion leading to equations (25) and (26), in the laboratory system we take the following representative values: $\ell_{0}^{(1)}=3 \mathrm{~cm}$, and $u_{0}^{(1)}=30 \mathrm{~km} / \mathrm{s}$. In the intergalactic medium, typical spatial and velocity scales for accretion shocks are $\ell_{0}^{(2)}=1 \mathrm{Mpc}$, and $u_{0}^{(2)}=1,400 \mathrm{~km} / \mathrm{s}$. This gives a scaling constant $g_{a} / g_{b}=2.2 \times 10^{22}$ for time variables, and thus $1 \mu \mathrm{s}$ in the laboratory translates to $0.7 \mathrm{Gyr}$ in for accretion shocks. Since $B=m_{i} \Omega / e$ and the vorticity scales as the inverse of time, then the laboratory results indicate that curved intergalactic shocks in protogalactic structures, with changing curvature at the level of a few tens of a percent on scales $\sim 1$ Mpc can generate magnetic field with values $\sim 10^{-21} \mathrm{G}$. This confirms for the first time in a direct experimental setting, the numerical estimates of Ref. [41].

\section{Laboratory Experiments with resistive return currents}

The generation of magnetic fields by resistive return currents is a process which is well know in laser-based experiments. As a matter of fact, this is an example where the laboratory work has provided a significant input to the understanding of astrophysical processes. The equations that describe the forward CR propagation in the intergalactic medium, in a laser-produced plasmas, are replaced by those of energetic electrons that stream across a solid slab of material illuminated, on one side, by a high intensity laser beam (Figure 9). Near the laser focus a density profile is set up and the laser energy is mostly deposited near or below the critical density [2], i.e. the density at which the laser frequency resonates with the plasma frequency $\omega_{\text {pe }}$. At low laser intensities $\left(\lesssim 10^{14}-10^{15} \mathrm{~W} / \mathrm{cm}^{2}\right)$ thermal electrons are generated near near the critical surface via inverse Bremsstrahlung. This coincides with the conditions in the experiments discussed previously. However, by operating the laser at much shorter pulse length, new regimes are accessible where electrons near the laser focus are directly accelerated by the ponderomotive force. In the case of an ideal plane wave geometry an electron, initially at rest, exposed to the electromagnetic field in the laser pulse, due to the conservation of the perpendicular component of the canonical momentum in a linearly polarised electromagnetic wave, at the end of the laser pulse its velocity returns to zero [109]. In a more realistic situation, the envelope of the laser pulse is not uniform having, for example, a Gaussian spatial profile, and, as a consequence, the electron feels a different electric field as it moves and thus subject to a net longitudinal force that pushes it towards the region of 

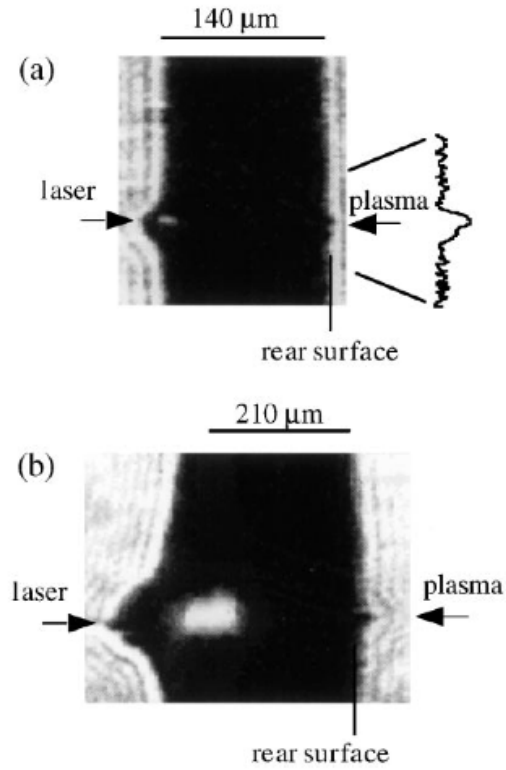

(c)

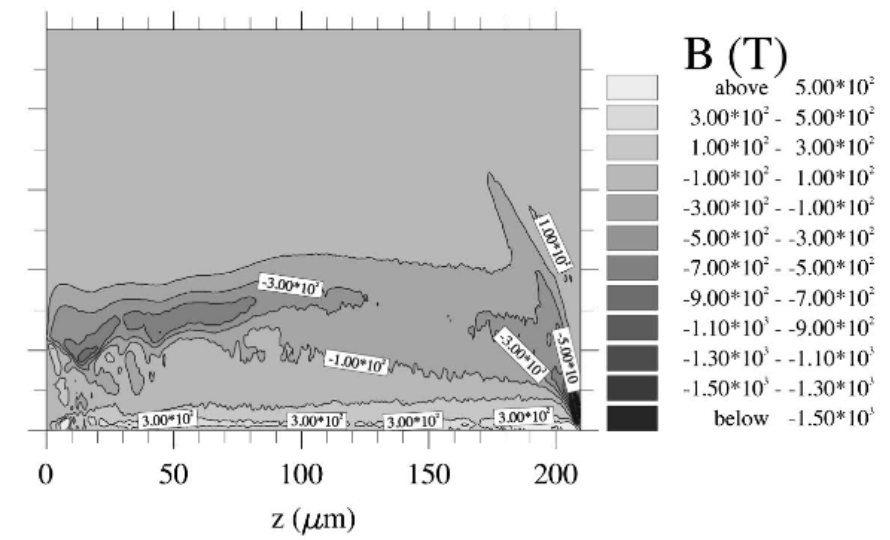

Figure 10: Left: Shadowgrams from laser experiment, showing clear evidence for collimation of fast electrons, for laser pulse duration and energy: (a) $22 \mathrm{ps}, 11.5 \mathrm{~J}$ (b) $207 \mathrm{ps}, 20.2 \mathrm{~J}$. The line in (a) gives a magnified trace of the rear surface of the region inaccessible to the probe. Adapted from Ref. [107]. Right: (c) Output from hybrid simulations showing contours of magnetic field strength at $3 \mathrm{ps}$, using $1 \mathrm{ps}, 20 \mathrm{~J}$ laser at $10^{19} \mathrm{~W} / \mathrm{cm}^{2}$. Adapted from [108].

lower laser intensity. This is the so-called "ponderomotive" acceleration [2]. At laser intensities $\gtrsim 10^{18} \mathrm{~W} / \mathrm{cm}^{2}$ the ponderomotive acceleration of electron is quite significant [110], and the inferred temperature from particle-in-cell simulations of the resulting fast electrons is [106]

$$
k_{B} T_{\text {fast }}=\left(\sqrt{1+\frac{I_{L} \lambda_{L}^{2}}{1.37 \times 10^{18}}}-1\right) m_{e} c^{2},
$$

where $I_{L}$ is the laser intensity in $\mathrm{W} / \mathrm{cm}^{2}$, and $\lambda_{L}$ the laser wavelength in $\mu \mathrm{m}$. Hence, electron temperatures in excess of $\mathrm{MeV}$ can be achieved, i.e. relativistic electrons. This is where we see the connection with cosmic ray streaming in the IGM. The fast-electrons in the experiment are partially collimated in the forward direction by the laser electromagnetic field, producing a current $j_{\text {fast }}$ in the target. The electrons in the over dense target will attempt to maintain charge neutrality, setting up a compensating current, $j_{\text {ret }}$, equal in magnitude but opposite in direction

$$
\nabla \times \boldsymbol{B}=\mu_{0}\left(\boldsymbol{j}_{\text {fast }}+\boldsymbol{j}_{\text {ret }}\right) \approx 0
$$

We see that this is analogous to the resistive mechanism suggested by [98] discussed in section 3.2, with the fast electrons taking the role of CR particles. The electrons that constitute the return current are again highly collisional, and a resistive electric field must exist to allow the electrons to overcome the frictional drag of the heavier joins in the target. This has been shown to play an important role in inertial confinement fusion (ICF) or fast ignition experiments, due to the resistively-generated magnetic fields causing self-focussing, cavitation or even shock wave formation in the target [108, 97, 111]. As an aside, it is interesting to note that, if the background plasma carrying the return current is highly magnetised $\omega_{g} \tau \gg 1$, as might be the case for example in the interstellar medium, the return current results in a very rapid magnetic field amplification process that is believed to be vital for acceleration of cosmic rays at supernova shocks $[112,113,114]$.

Using the same estimates as those given in Eq. (12), the inferred magnetic field in the experiments is of the order of tens on MG or higher. This has been confirmed in numerical simulations [108,97] as well as in experiments [107]. 
Figure 10 shows comparison between experiments and numerical experiments, demonstrating the self generation of magnetic fields, and focussing of electrons. These were obtained from a series of experiments at the Vulcan laser at Rutherford Appleton Laboratory [115] by using a high intensity beam. Thanks to the method of chirped pulse amplification (CPA) of optical laser light [116], nanosecond laser pulses can be compressed in time to sub-picosecond durations, yet carrying hundreds of joules of laser energy. In a glass laser system as the one shown in Figure 2, the maximum laser energy is limited by the damage threshold of the gain medium. The CPA technique circumvents this problem by using a pair of gratings that introduce a temporal chirp, i.e., meaning that different wavelengths travel a different distance between the gratings, and so the pulse is stretched in time. By reducing the thermal load on the gain medium, the stretched laser pulse can be further amplified. Then, prior to delivery to the target, a second pair of gratings is used to recompress back in time the amplified laser pulse. State-of-art laser systems can now achieve focused intensities exceeding $10^{20} \mathrm{~W} / \mathrm{cm}^{2}$.

\section{Laboratory Experiments of Weibel's Instability}

While the Weibel instability is thought to play a role in many laboratory situations, there are now a number of dedicated campaigns to explore specifically the role of Weibel instability in shock mediation. The experimental approach, pioneered in the papers $[117,118]$, involve generating a fast interpenetrating plasma flow by irradiating two oppositely facing foils with high-power lasers. Fig. 11 shows the results of a more recent experiment [119]. The two counter-streaming plasma flows are obtained in this case by irradiating a pair of plastic $(\mathrm{CH})$ targets, separated by 4.5 $\mathrm{mm}$, with a $1.8 \mathrm{~kJ}, 2 \mathrm{~ns}$ laser pulse at a wavelength of $351 \mathrm{~nm}$ (the UV beams in Fig. 11). The electromagnetic fields formed in the interaction region were probed using a proton radiography diagnostics [120, 121]. The high energy protons were generated with a third, high-intensity laser pulse $(1,053 \mathrm{~nm}, 800 \mathrm{~J}, 10 \mathrm{ps}$, referred to in the diagram as the IR beam in Fig. 11) focused to $10^{18} \mathrm{~W} / \mathrm{cm}^{2}$, irradiating a thin $\mathrm{Cu}$ disk $8 \mathrm{~mm}$ from the interaction region. This creates a uniform and laminar point source of protons, with a distribution of energies of order $10 \mathrm{MeV}$ via the targetnormal sheath acceleration mechanism [122, 123]. A stack of radiochromic film (RCF) $80 \mathrm{~mm}$ from the interaction region was used to detect the protons with a geometrical magnification of 11. Fig. 11 also suggests the formation of magnetic structures produced via the Weibel instability, with the size of the filaments on the order of the ion skin depth. It remains unclear however, whether coherent magnetic structures on cosmological scales can emerge out of the initial microscopic ones initialised on the ion skin depth, although collisions can play an important role. Perhaps, as in the case for the Bierman produced seed fields, mixing and stretching of the small scale fields by turbulence in the flow may assist. In this respect, the interacting plume experiments of Ref. [124] seems to suggest that large-scale self-organized plasma structures can evolve in presence of a disturbed hydrodynamic flow, although the resulting structures were coherent.

More recent work based on the same platform as the one of Fig. 11 [125] shows that the spacing of the Weibel filaments increases with time (see Fig. 12), an indication that the magnetic field is growing and increasing the deflection of the probe proton beam [125]. The larger magnetic field indicates the efficiency of the instability to convert kinetic energy into magnetic energy. The magnetic energy associated with the instability is driven by the ion flows, and goes mainly into the transverse component of the field. Numerical simulations using the code Osiris [126], see Figure 12, produce an initial linear amplification phase that saturates after 1-1.5 ns. After this time, the field amplitude and filament size continue to increase but at a reduced rate reaching a plateau at about $1 \%$ conversion of the initial kinetic energy of the ions into magnetic energy. In these experiments, however, the interpeneration of the two conterstreaming flows occurred over a distance that was insufficient to reach a fully formed collisionless shock, thus limiting the upper level of the flow magnetization [125]. On the other hand, the very fact that an increased magnetization of the flow was observed is an important confirmation that the Weibel instability in an initially unmagnetized medium is capable of generating the percent-level magnetization, as observed in Gamma Ray Bursts; collisions of relativistic flows are likely to produce even stronger fields. This agrees with multiwavelength observations of afterglow emission of GRBs suggesting sub-equipartition levels of magnetic field in the region behind the forward shock [127].

\section{Magnetic field amplification by turbulence}

Magnetic-dynamo studies in the laboratory have to date been carried out primarily using liquid-metal experiments $[128,129]$ and only recently in plasmas [82] (see below), but in both cases the experiments have been performed in the 
(a)

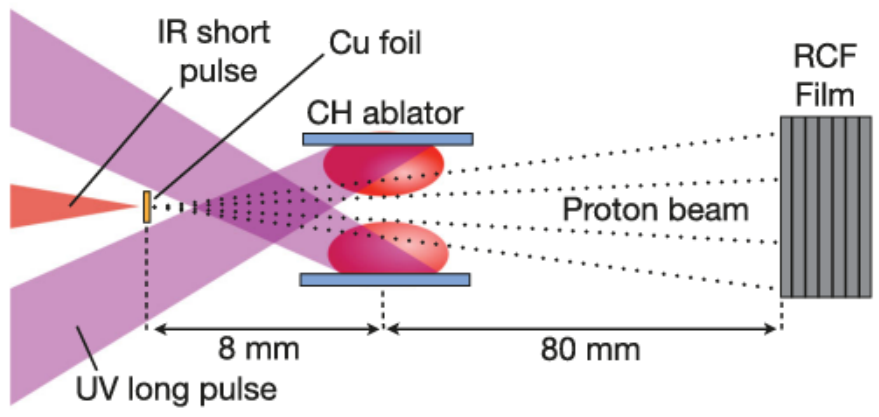

(b)

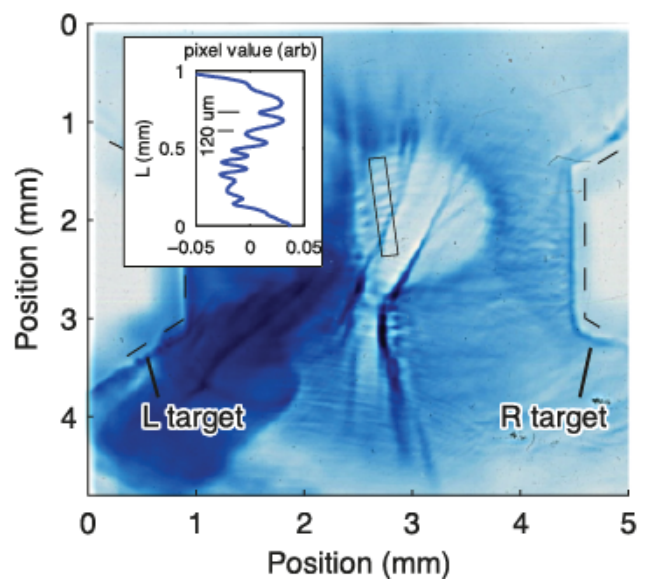

Figure 11: (a) Schematic of the experiment aimed at measuring Weibel-produced filaments. (b) Proton images of the field filaments at the collision point between the two plasmas. Adapted from Ref. [119].
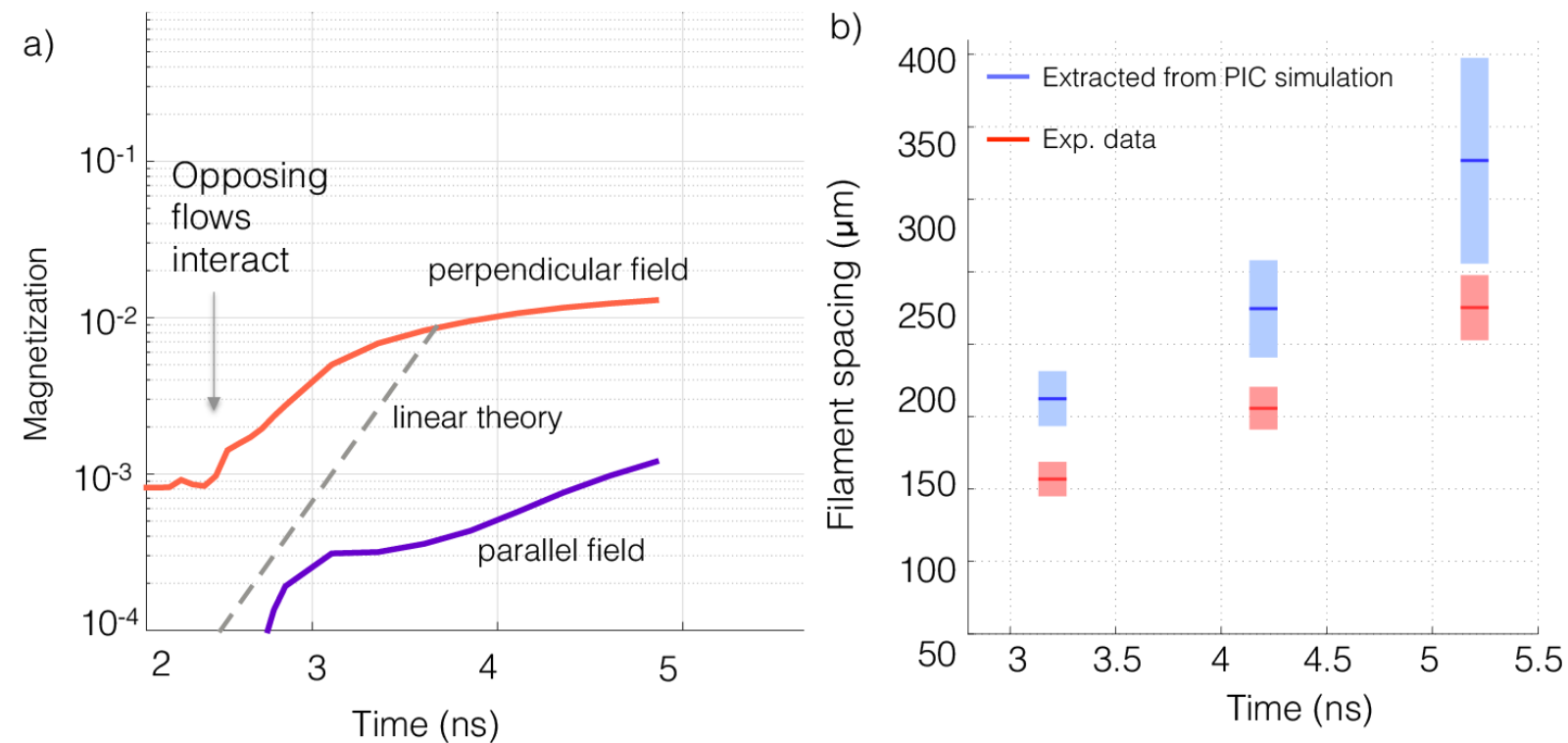

Figure 12: (a) Temporal evolution of the plasma magnetization $\left(B^{2} / 2 \mu_{0} \rho v^{2}\right)$ as a function of time. Due to the presence of Biermann generated magnetic fields near the laser spot, the perpendicular magnetization is $\sim 0.1 \%$ before the flows interact. After the collision oft he two flows, magnetic energy associated with Weibel instability increases sharply, by a factor greater than 10 in several ns. The magnetization due to the ion Weibel instability, growing at the theoretical linear growth rate is shown in the plot. This calculation shows that the Weibel-generated magnetization becomes the dominant contribution to the overall magnetization of the system. (b) Measurement of the mean separation between filaments in experimental proton radiographs (red) and synthetic proton images from 3D PIC simulations (blue). The filament spacing approximately doubles over the 2 ns of observation. Adapted from Ref. [125]. 
(a)

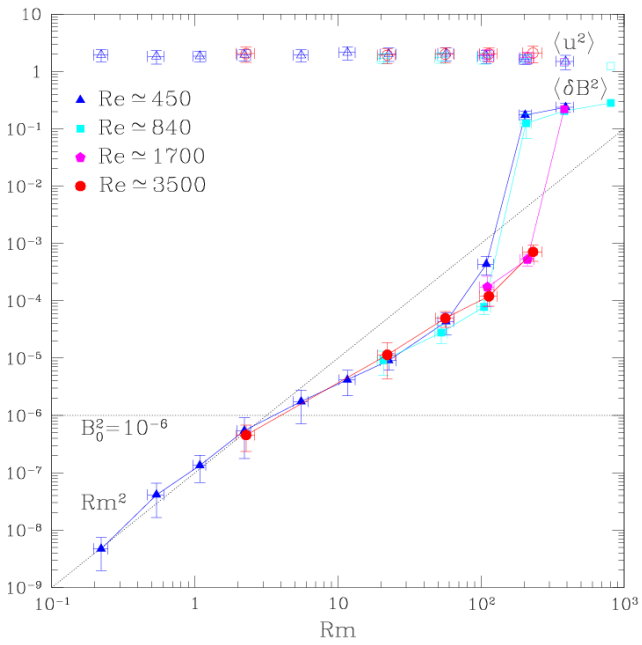

(b)

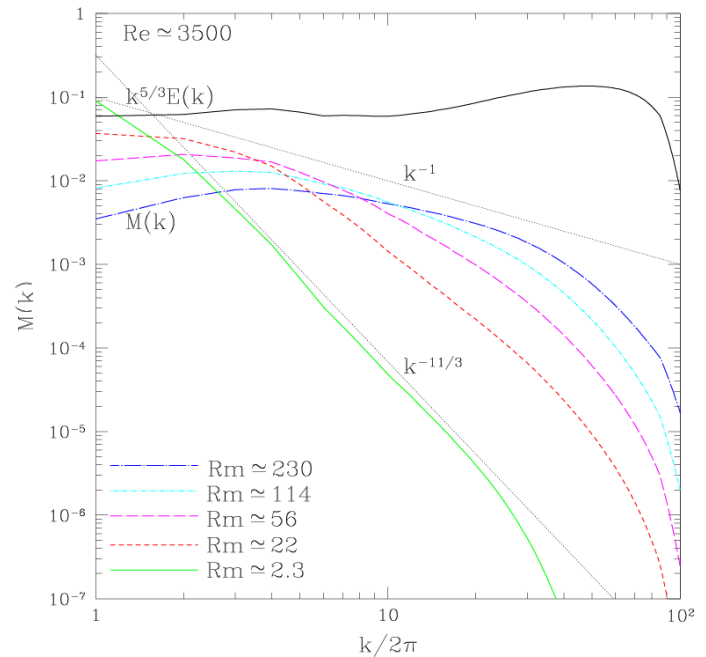

Figure 13: (a) Numerical simulations showing the growth of magnetic field as a function of Rm. (b) Calculated magnetic energy spectra. Adapted from Ref. [130].

regime where $R m \ll R e$, and magnetic diffusion dominates (i.e., $R m$ is much smaller compared to the astrophysical case). In order to understand magnetic field amplification, let's consider the induction equation and write $\mathbf{B}=\mathbf{B}_{0}+\delta \mathbf{B}$, where $\mathbf{B}_{0}$ is the small seed produced by the Biermann battery process. The linearized dimensionless induction equation reads [130]

$$
\frac{\partial \delta \mathbf{B}}{\partial t}+\mathbf{u} \cdot \nabla \delta \mathbf{B}=\delta \mathbf{B} \cdot \nabla \mathbf{u}+\frac{1}{R m} \nabla^{2} \delta \mathbf{B}+\mathbf{B}_{0} \cdot \nabla \mathbf{u} .
$$

If $R m$ is small, then the diffusion term dominates and

$$
\frac{1}{R m} \nabla^{* 2} \delta \mathbf{B} \approx-\mathbf{B}_{0} \cdot \nabla \mathbf{u},
$$

or,

$$
\eta \nabla^{2} \delta \mathbf{B} \approx-\mathbf{B}_{0} \cdot \nabla \mathbf{u},
$$

and thus $|\delta \mathbf{B}|^{2} \approx R m^{2}\left|\mathbf{B}_{0}\right|^{2}$. Taking the spatial Fourier transform of (37) we get $\eta k^{2} \delta \mathbf{B}_{\mathbf{k}}=i\left(\mathbf{k} \cdot \mathbf{B}_{0}\right) \mathbf{u}_{\mathbf{k}}$, which implies

$$
M(k)=\frac{B_{0}^{2}}{3 \eta^{2}} \frac{E(k)}{k^{2}},
$$

where $M(k)=\left|\delta \mathbf{B}_{\mathbf{k}}\right|^{2} / 3$ is the one-dimensional spectrum of the magnetic field fluctuations, and $E(k)=\left|\mathbf{u}_{\mathbf{k}}\right|^{2}$ the kinetic energy spectrum. In the case of Kolmogorov turbulence, $E(k) \sim k^{-5 / 3}$, thus $M(k) \sim k^{-11 / 3}$ (the Golitsyn spectrum), characteristic of magnetic fluctuations at low magnetic Reynolds number [131, 132]. With a small magnetic Reynolds number, the amplification is due to the stochastic tangling of an imposed field, $B_{0}$, by turbulent motions, and the saturated level is set by balancing this effect with Ohmic diffusion. In this regime, the magnetic field grows proportionally to the imposed (seed) field, as shown in Fig. 13. As $R m$ gets larger, the magnetic spectrum becomes flatter (see Fig. 13) and total amplified field grows as $R m^{a} B_{0}$, with $a<1$, as seen in MHD simulations [130]. It remains unclear, for example, what ultimately sets the saturated levels of the magnetic field. If the magnetic Reynolds number gets even larger, Eq. (37) ceases to be valid, and the fluctuation dynamo sets it. When $R m \gg 1$, the leading contribution in (35) gives $\left|\delta \mathbf{B}_{\mathbf{k}}\right|^{2} \approx\left|\mathbf{B}_{0}\right|^{2}$, and thus $M(k) \sim B_{0}^{2} k^{-1}$ [133]. The saturated state is determined by the back reaction of the fluid, and the magnetic energy density reaches equipartition with the turbulent kinetic energy (see Fig. 13). This is indeed what is needed to explain the large scale magnetization in galaxies and clusters of galaxies. 


\section{Turbulent plasma flow in the laboratory}

The idea of testing magnetic field amplification in laboratory plasmas is thus compelling. The high Reynolds number flows produced in the laser experiments described previously are expected to be naturally turbulent. This opens up the possibility of exploring turbulent induction, or dynamo, in laser produced plasmas. In the context of the Biermann battery generated fields at shocks, this can also be seen as an attempt to investigate the second stage of galactic magnetic field generation [134]. Unfortunately, the magnetic Reynolds numbers currently achievable in the laboratory are quite modest. In an effort to accelerate this process, [82] investigated the effect of passing a laser produced shock through a grid, to enhance the vorticity, and this increase the rate of stretching of magnetic fields. The experiment, shown in Fig. 14 uses the same platform described in Fig. 3 (thus having a shock and self-generated magnetic fields), but then disrupting the shock curvature by interaction with strong density variations. This mimics the process of astrophysical turbulence driven by shocks interacting with a clumpy interstellar medium. Alternatively, one can see this as a mean-field dynamo experiment, where motivated by the measurements of large scale magnetic field in the absence of a grid, introduction of a small scale fluctuating velocity field should increase the ratio of the two terms in the induction equation, namely $\nabla \times(\boldsymbol{u} \times \boldsymbol{B})$ and $\frac{\eta}{\mu_{0}} \nabla^{2} \boldsymbol{B}$.

In this experiment, the chamber is filled with argon gas at $P=1 \pm 0.2$ mbar. This has advantages compared to helium as spectroscopic measurements of the plasma temperature become available. One the other hand, the heavier ions make the flow slower. Three frequency doubled (527 nm wavelength) laser beams of the Vulcan laser facility are focussed onto a $500 \mu \mathrm{m}$ diameter carbon rod with a laser spot diameter of $300 \mu \mathrm{m}$. The total laser illumination is $300 \pm 30 \mathrm{~J}$ in a $1 \mathrm{~ns}$ pulse. The corresponding laser intensity is $4 \times 10^{14} \mathrm{~W} / \mathrm{cm}^{2}$. As done for the experiment on Biermann battery discussed earlier, the shock wave evolution was monitored using transverse interferometry and Schlieren with an optical probe (with $532 \mathrm{~nm}$ wavelength and $5 \mathrm{~ns}$ gate width). The interferometer was of Mach-Zehnder type with $\sim 50 \mathrm{~mm}$ field of view, and was used to provide the electron density. The horizontal mid-plane joining the carbon rod position and the tip of the magnetic induction coils was also imaged onto a $50 \mu \mathrm{m}$ slit of a visible spectrometer coupled to a gated CCD camera (with $20 \mathrm{~ns}$ gate). This provided space and time dependent temperature information.

The interaction of a shock with large density perturbations is reproduced in the laboratory by passing a laser produced shock in a plasma through a plastic mesh with $\ell=1.1 \mathrm{~mm}$ grid cell size, and $0.4 \mathrm{~mm}$ thick wires (see Fig. 14). The turbulent flow is clearly visible in Fig. 14. However, we should note that the Schlieren imaging technique measures density gradients, thus turbulent flow, with less distinct gradients, exhibits a reduced contrast. Without the presence of the grid the shock closely follows a Sedov-Taylor solution [93, 93] with $R_{s} \propto t^{2 / 5}$, as indicated in Fig. 15. The estimated maximum error of $\pm 2 \mathrm{~mm}$ in the experimental position is due to the uncertainty in the optical images to define the exact location of the shock front. The inferred electron density at the shock front is $n_{e} \sim 2 \times 10^{17}$ $\mathrm{cm}^{-3}$. The measured spectral lines at $1 \mathrm{~cm}$ from the carbon rod position (averaged over $0.2 \mathrm{~cm}$ ) are also shown in Fig. 15. The ratio of the emission lines has been fitted with the collisional-radiative code PrismSPECT [135]. In this density regime, the line ratios are weakly dependent on the electron density, thus providing a good measurement of the electron temperature $\left(T_{e}\right)$. The error in the reported temperature values has been estimated from the small variations in the calculated spectral line shapes with changes in $T_{e}$ of $\pm 0.5 \mathrm{eV}$. Numerical simulations performed in $1 \mathrm{~d}$ spherical geometry with the collisional-radiative hydrodynamic code HELIOS-CR, reproduce well the shock position, peak electron density and temperature values (see Fig. 15).

At the position of the grid $\left(R_{s 0}=1 \mathrm{~cm}\right)$, the flow velocity of the shock is measured to be $20 \mathrm{~km} / \mathrm{s}$ (Mach number $\sim 9$ ), although this increases upon interaction with the grid. The Reynolds number corresponding to this shock radius is $R e \sim 5 \times 10^{4}$, (with $v \approx 40 \mathrm{~cm}^{2} / \mathrm{s}$ ) while the magnetic Reynolds number is $R m \sim 3-6$ (with $\eta \approx 4 \times 10^{5} \mathrm{~cm}^{2} / \mathrm{s}$ ), and the Biermann number is $B i \sim 6$. The shock is observed to arrive at the position of the grid at $t \sim 0.3 \mu \mathrm{s}$. With the intention to describe the same astrophysical accretion shock structure as in the experiment of Fig. 3, i.e., $\ell_{0}^{(2)}=1 \mathrm{Mpc}$, and $u_{0}^{(2)}=1,400 \mathrm{~km} / \mathrm{s}$, in the present experiment we have $\ell_{0}^{(1)}=1 \mathrm{~cm}$, and $u_{0}^{(1)}=20 \mathrm{~km} / \mathrm{s}$, thus $g_{a} / g_{b}=4.4 \times 10^{22}$, and $0.3 \mu$ s corresponds to 0.4 Gyr. Similarly, the same scaling relation implies that the density perturbation of $\sim 1.1$ $\mathrm{mm}$ in the laboratory, corresponds to $\sim 110 \mathrm{kpc}$ in the intracluster medium.

\section{Measurements of turbulent induction}

The main goal of the experiment of Fig. 14 was, of course, the measurement of magnetic fields. As before, these measurements were performed with an induction coil, but consisting of 4 twisted pair coils wound around the axis of 


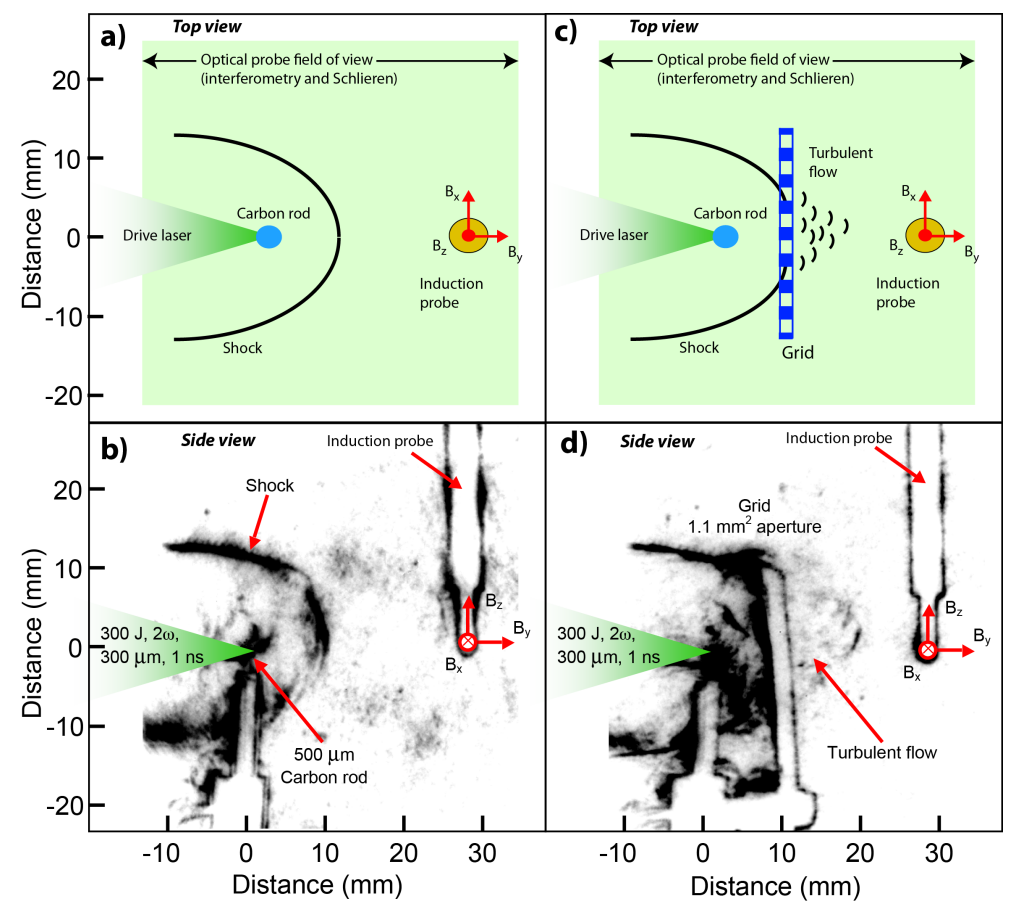

Figure 14: (a) Diagram of the experiment without the grid. (b) Same as (a) but in the presence of a plastic grid placed at $1 \mathrm{~cm}$ from the carbon rod position. (c) Schlieren image at $t=300 \mathrm{~ns}$ after the laser shot and without the grid. (d) Same as (c) but with the grid. From Ref. [82].
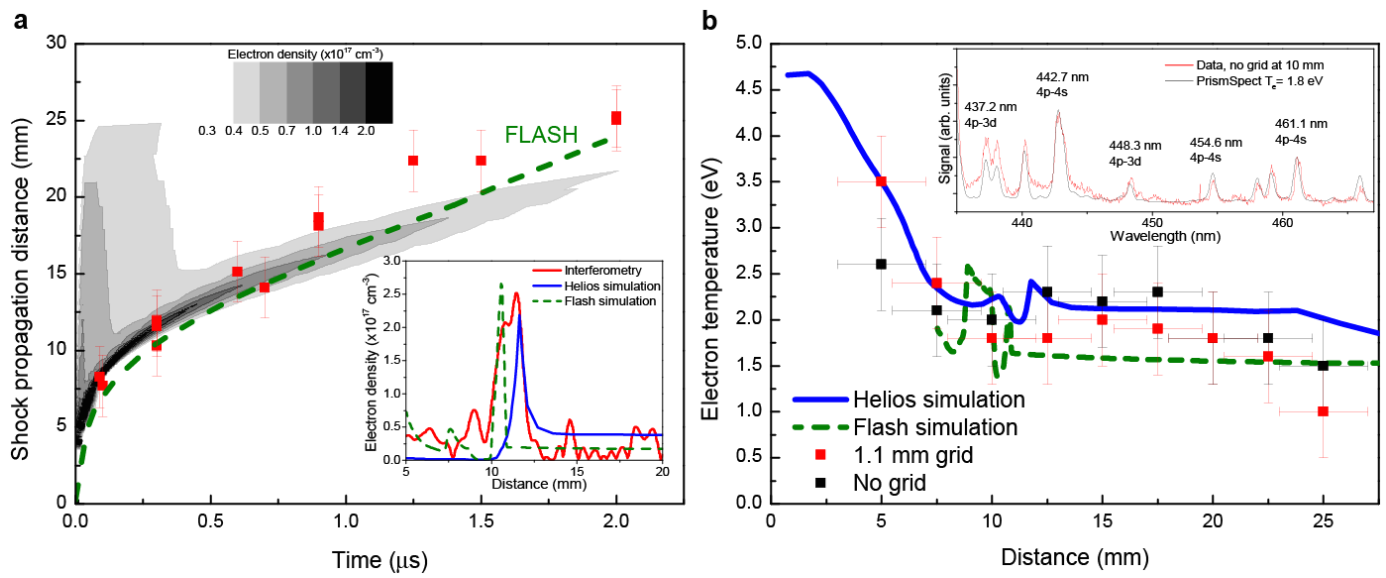

Figure 15: (a) Measurement of the shock wave position $v s$ time in the absence of a grid obtained from Schlieren data (red squares), 1d HELIOS-CR simulations (contours) and 2d FLASH simulations (dashed green line). The inset shows the measured density profile obtained via interferometry at $t=300 \mathrm{~ns}$ compared with simulation predictions. The estimated maximum error of $\pm 2 \mathrm{~mm}$ in the experimental position is due to the uncertainty in the optical images to define the exact location of the shock front. (b) Measured $T_{e}$ profiles at $t=300 \mathrm{~ns}$ without (black symbols) and with (red symbols) a grid obstacle in the flow. The low value of $T_{e}$ near the target in the FLASH simulation is an artifact due to expansion of the cold carbon material; we therefore omit this part in the graph. The inset shows the measured spectral lines at $1 \mathrm{~cm}$ from the carbon rod position. From Ref. [82]. 

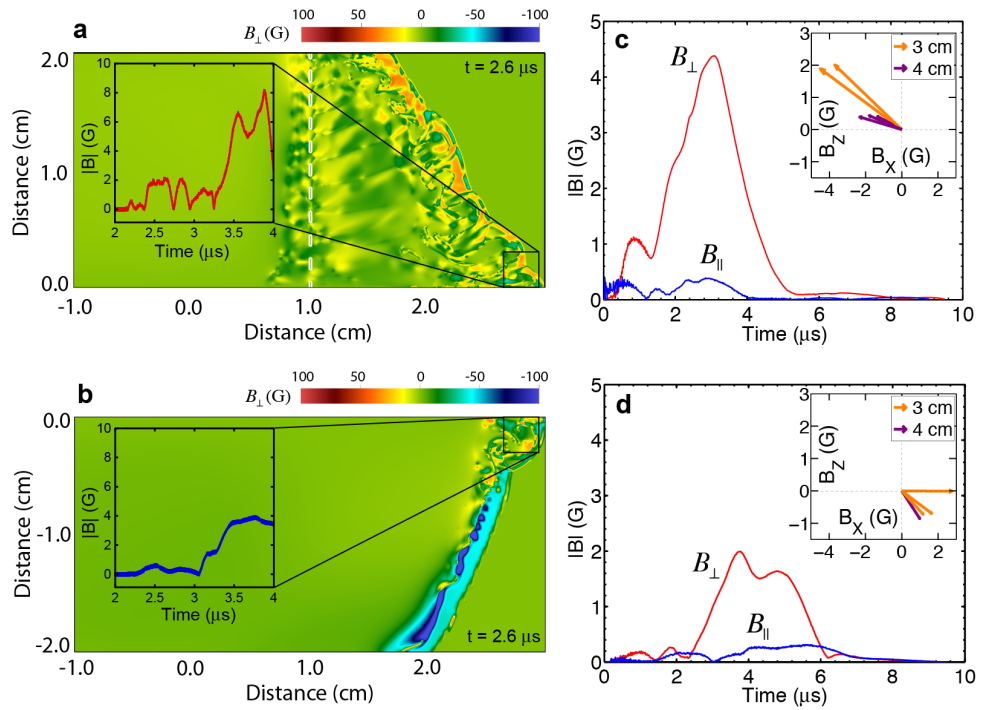

Figure 16: (a) FLASH simulation of the shock propagation through a grid at $t=2.6 \mu$ s. The simulation was initialized with circular target (of radius $250 \mu \mathrm{m})$ of carbon in the origin of a [0,2 cm] $\times[-1,3 \mathrm{~cm}]$ cylindrical $(r-z)$ computational domain, which is filled with argon gas at $1 \mathrm{mbar}$. The target is illuminated by a $300 \mathrm{~J}$ laser pulse for $1 \mathrm{~ns}$, with a spot size of $300 \mu \mathrm{m}$. The inset shows the simulated magnetic field at the induction probe location. (b) Same as (a) but with no grid. (c) The magnetic field measured at $3 \mathrm{~cm}$ from the carbon rod with components along the axis as shown in Fig. 14 for the grid case. The inset shows the projection of the magnetic field onto the $x$ - $z$ plane at the time of its maximum intensity. Each vector corresponds to different shots. Data obtained at a distance of $4 \mathrm{~cm}$ from the carbon rod are also shown in the inset. In the latter case, the field magnitude is considerably reduced. (d) Same as (c) but without the grid. From Ref. [82].

a smaller, $\sim 1 \times 1 \mathrm{~mm}^{2}$, plastic core. The coils were protected from the surrounding plasma by a boron-nitride tube, instead of a glass tube as shown in Fig. 6. With this design a time resolution of $10 \mathrm{~ns}$ was achieved. Wind tunnel experiments with a steady flow have shown that homogeneous turbulence is well developed at distances of 20 times the grid cell size [136], and this motivates to take magnetic field measurements predominantly at a distance of $2 \mathrm{~cm}$ (and $3 \mathrm{~cm}$ in some cases) downstream of the grid, which is where the induction probe was placed.

Magnetic field measurements with and without the grid are shown in Fig. 16, together with MHD simulations. The simulations were performed using FLASH [78, 137], a finite-volume MHD, shock capturing code that has recently been extended to model laboratory experiments. The simulations were performed in 2D cylindrical geometry. In order to model the full range of physical processes involved in the experiments, the simulations included the unsplit staggered mesh MHD solver [138], extended to three temperatures and cylindrical geometry, explicit Spitzer resistivity, implicit thermal conduction and heat exchange, as well as multi-group radiation diffusion with multi-material tabulated opacities and equations of state $[139,140]$.

In the case of a grid, the measured perpendicular component $\left(B_{\perp}^{2}=B_{x}^{2}+B_{z}^{2}\right.$ in Fig. 14) of the magnetic field is 2-3 times larger than without it. The time evolution of the magnetic field at the position of the induction probe is also well reproduced by the simulations. The calculated magnetic field traces at $2.8 \mathrm{~cm}$ from the grid position along the symmetry axis, averaged over a $3 \mathrm{~mm}$ x $3 \mathrm{~mm}$ volume, are also shown in Fig. 16, both with and without a grid. We notice that the measured signal starts earlier in the case with a grid than in the case without a grid. This likely occurs for two reasons. First, the shock is accelerated as it passes through the grid. As shown in earlier hydrodynamic simulations [50] and in FLASH simulations of the experiment, for large Re, the grid acts as a channel, which accelerates the flow as it passes through the pores, after which the flow velocity decreases. This can be seen in Fig. 14, where, with the grid, the turbulent flow seems to advance more rapidly towards the position of the magnetic field diagnostics. From Schlieren imaging, for all experiments with the grid, the shock is observed to arrive at the position of the probe at $t \gtrsim 1.5 \mu \mathrm{s}$. Second, the grid shadows the gas beyond it from the high-temperature radiation emitted at early times. Thus, in the case with a grid a significant fraction of the gas beyond the grid is not heated by the radiation produced as the laser hit the carbon target, and so has a higher diffusivity. This effect is visible in the 


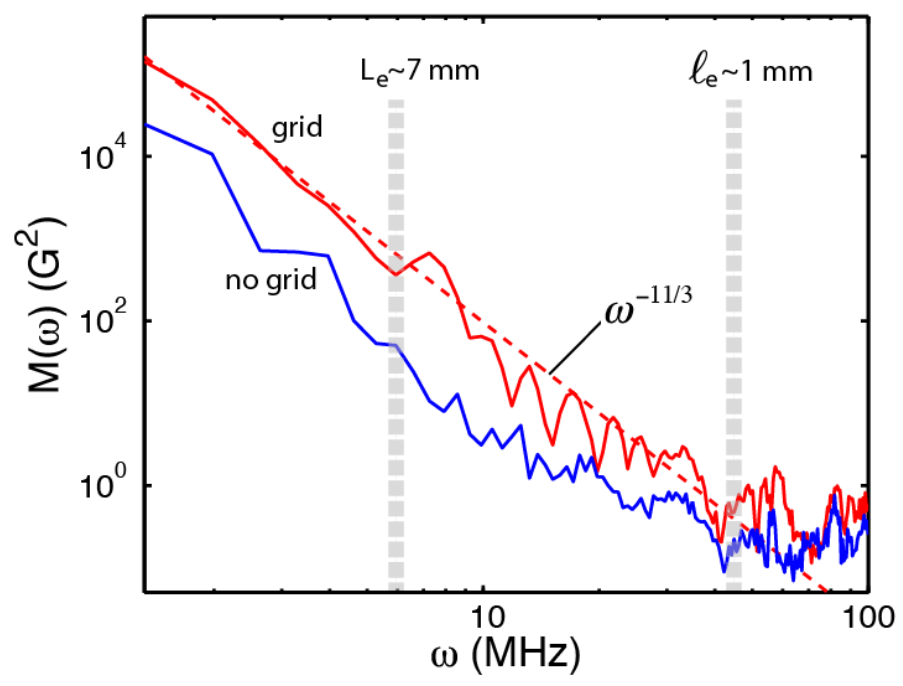

Figure 17: Plot of the measured magnetic energy spectrum $M(\omega)$. From Ref. [82].

measured temperature profiles of Fig. 15.

Fig. 17 shows the plot of the measured magnetic energy spectrum $M(\omega)=|B(\omega)|^{2}$, where $B(\omega)$ is the discrete Fourier transform of the total magnetic field as a function of time, as measured by the induction probe. $M(\omega)$ is obtained by averaging all the available laser shots with the induction coils placed at $3 \mathrm{~cm}$ from the carbon rod position. The measured spectrum shows a power law $\omega^{-11 / 3}$. Since the mean flow velocity is larger than the (subsonic) velocity fluctuations excited by the grid, then, according to Taylor's hypothesis [141], the frequency of the fluctuations is proportional to $k$, the wavenumber of the magnetic fluctuations. The magnetic energy spectrum thus exhibits the Golitsyn $\left(k^{-11 / 3}\right)$ power law dependence [131], expected for small magnetic Reynolds numbers. The experiment show that the downstream flow velocity is disturbed. The vorticity induced as the shock overtakes the grid acts as a seed for turbulent generation of magnetic field on smaller scale. Beyond the grid, the vorticity is stretched in the direction of the flow, so that the spatial scales of the largest eddies is $L_{e} \sim 7 \mathrm{~mm}$ by the time the shock reaches the induction probe (see Fig. 16). The flow velocity at the position of the grid is $v_{e} \sim 7 \mathrm{~km} / \mathrm{s}$, with the inferred energy-containing scale given by $L_{e}$. This scale determines the energy injection into the turbulent cascade, as shown in Fig. 17. The resolution limit on the high frequency side is instead determined by the coil size $\left(\ell_{e} \sim 1 \mathrm{~mm}\right)$. FLASH simulations without a grid show that the shock becomes unstable when it is overtaken by the compositional discontinuity between the vaporized carbon target material and the gas, generating vorticity, and therefore magnetic fields, at spatial scales $\lesssim 3 \mathrm{~mm}$. This is the origin of the $k$-spectrum of the magnetic field without a grid. Simulations with a grid present show that the size of the largest eddies is $\sim 2$ times larger, the flow velocity is $\sim 50 \%$ larger, and thus the magnetic Reynolds number is $\sim 3$ times larger. This is consistent with the higher magnetic field seen in the experimental data. This thus supports the mechanism of field amplification by turbulent induction: the amplified field is proportional to $R m$, and it remains dynamically unimportant since the Alfvén speed $\left(v_{A} \sim 400-1,400 \mathrm{~cm} / \mathrm{s}\right)$ is smaller than the flow velocity.

FLASH simulations are also used to confirm that the observed magnetic fields are the results of amplified Biermann battery seeds produced near the the grid by the shock wave, and not due to the diffusion of large magnetic fields generated as the laser hits the carbon target. Indeed, at the laser spot, due to small spot size and the large amount of deposited energy, large temperature and density gradients are established and those can drive Biermann battery to generate megagauss magnetic fields [50]. These are seen in the FLASH simulations as well, and so it is important to separate them from the smaller fields produced by the Biermann process at the shock. Fig. 18 shows FLASH simulations with the Biermann battery term turned on only during the first $30 \mathrm{~ns}$ and switched off afterwards. Comparison with a simulation which had baroclinic generation present throughout the simulation thus indicates the magnetic field is indeed enhanced as the shock passes through the grid, mainly due to the induced turbulence. 

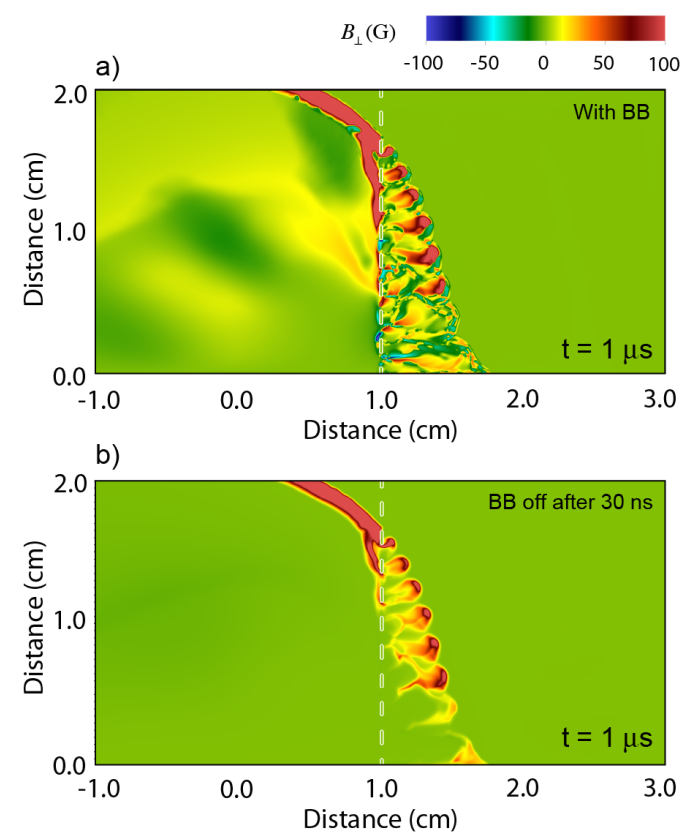

Figure 18: (a) FLASH simulations of the shock propagation with the Biermann battery (BB) term included. (b) Same as (a) but with the Biermann battery term omitted except for the first 30 ns. From Ref. [82].

\section{Magnetic field growth rate}

The results presented in the previous section, with the magnetic field spectrum exhibiting the Golitsyn power law, suggests Kolmogorov turbulence. In the regime where $R e \gg R m$ (as in the experiments discussed here), velocity fluctuations on a scale $\ell$ are thus $u_{\ell} \sim(\epsilon \ell)^{1 / 3}$, where $\epsilon$ is the power injected into the turbulence, which, in Kolmogorov turbulence, is assumed constant in the inertial range. The rate of stretching of the magnetic field at the scale $\ell$ is $u_{\ell} / \ell \sim \epsilon^{1 / 3} \ell^{-2 / 3}$. The resistive scale, i.e., the scale at which resistive diffusion become important, can be determined by equating the stretching rate with the diffusion rate, $\eta / \ell^{2}$. This gives [130]

$$
\ell_{\eta} \sim\left(\frac{\eta^{3}}{\ell}\right)^{1 / 4} \sim \frac{L_{e}}{R m^{3 / 4}}
$$

where $L_{e}$ is the energy containing (outer) scale and $R m$ has been evaluated at the outer scale. The growth rate of the magnetic field is thus

$$
\gamma \sim \frac{u_{\ell_{\eta}}}{\ell_{\eta}} \sim\left(\frac{\epsilon}{\eta}\right)^{1 / 2} \sim \frac{u_{e}}{L_{e}} R m^{1 / 2},
$$

where $u_{e}$ is the fluid velocity at the outer scale. Because of the dependence with $R m$, in the case of large magnetic Reynolds numbers this dynamo is faster than any other dynamo associated with the outer scale motions (which would growth at rate $\sim u_{e} / L_{e}$ ).

In oder to estimate the grow rate in the experiment, let's consider the magnetic field measured at two different points in space. Let $B_{1}(t)=\left(B_{x 1}^{2}+B_{y 1}^{2}+B_{z 1}^{2}\right)^{1 / 2}$ be the field measured at $3 \mathrm{~cm}$, and $B_{2}(t)=\left(B_{x 2}^{2}+B_{y 2}^{2}+B_{z 2}^{2}\right)^{1 / 2}$ the field at $4 \mathrm{~cm}$ from the carbon rod position. We define the cross correlation function between these two points as:

$$
C_{B B}(t)=\frac{1}{T_{2}-T_{1}} \int_{T_{1}}^{T_{2}} B_{1}(s) B_{2}(t+s) d s
$$




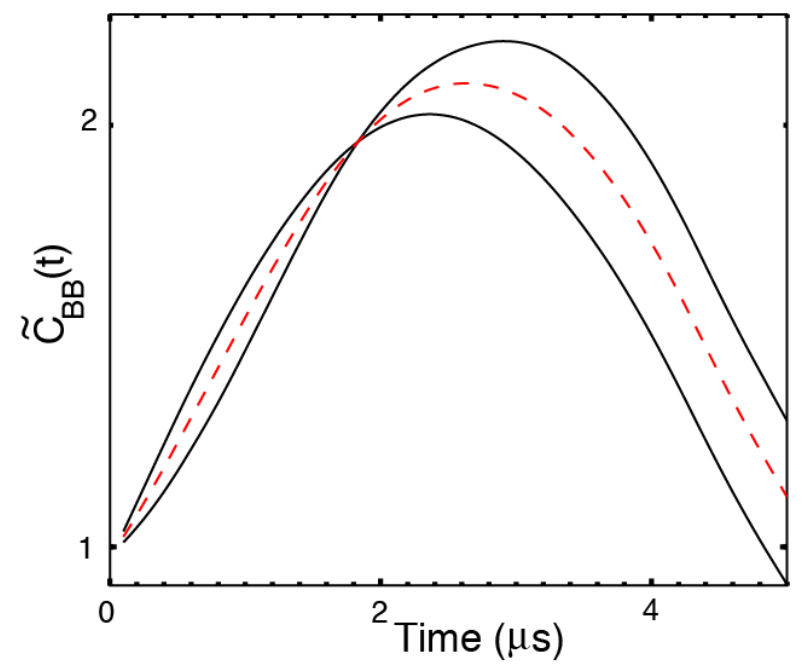

Figure 19: Plots of the normalized two-point cross correlation function, $\tilde{C}_{B B}(t)$, of the measured magnetic field at $2 \mathrm{~cm}$ and $3 \mathrm{~cm}$ from the grid position. From Ref. [82].

where $T_{1}$ and $T_{2}$ are the initial and final sampling times, respectively. In our case $T_{1}=2 \mu \mathrm{s}$ and $T_{2}=7 \mu \mathrm{s}$. We rewrite the induction equation (4) as

$$
\frac{d \mathbf{B}}{d t}=\frac{\partial \mathbf{B}}{\partial t}+\mathbf{u} \cdot \nabla \mathbf{B}=\mathbf{B} \cdot \nabla \mathbf{u}+\eta \nabla^{2} \mathbf{B}
$$

where we have assumed an incompressible fluid. The first term on the right side of (42) describes the growth of magnetic field due to stretching and twisting of the field lines, and this operates, on a spatial scale $\ell$, with a characteristic rate $\gamma \sim u_{\ell} / \ell$. The second term in (42) represents the resistive dissipation, with a characteristic decay rate given by $\eta / \ell^{2}$. Eq. (42) can be rewritten in scalar form as

$$
\frac{d B}{d t} \sim\left(\gamma-\frac{\eta}{\ell^{2}}\right) B=\tilde{\gamma} B
$$

where $\tilde{\gamma}$ is an effective growth (or decay) rate. We take $\tilde{\gamma}$ to be independent of time, which essentially limits our analysis to times before saturation has been reached. We have $B_{2}(t+u)=B_{2}(u) \exp (\tilde{\gamma} t)$, and

$$
\tilde{C}_{B B}(t)=\left[\frac{C_{B B}(t)}{C_{B B}(0)}\right]=\exp (\tilde{\gamma} t) .
$$

The increase of the normalized correlation functions is then a measure of the effective growth rate. This function is plotted in Fig. 19. Thus the slope of the normalized two-point cross correlation function for times $\lesssim 2 \mu$ s gives an amplification rate $\tilde{\gamma}=4 \times 10^{5} \mathrm{~s}^{-1}$. Since the separation between the two points where the magnetic field is measured is comparable to the outer scale, estimating $\ell \sim L_{e}$, we have $\eta / \ell^{2} \approx 8 \times 10^{5} \mathrm{~s}^{-1}$ and thus $\gamma \sim 1.2 \times 10^{6} \mathrm{~s}^{-1}$. This timescale gives a good order-of-magnitude estimate of the effective stretching rate of the chaotic flow behind the grid as predicted by Eq. (40). From our scaling relations, this laboratory stretching rate corresponds to a growth rate of $0.9 \mathrm{Gyr}^{-1}$ in the intracluster plasma. On the other hand, this rate only represents the minimal rate of magnetic field amplification one should expect in an astrophysical plasma, where resistivity is low and Rm is large. At smaller scales in our experiment, where resistivity dominates, there are still sustained magnetic fluctuations (see Fig. 17). The timescale for the resistivity-chaotic-tangling balance to be established at those scales is $\eta / \ell_{e}^{2}$, where $\ell_{e}$ is of order 1 $\mathrm{mm}$ (which is the induction coil size, therefore the measurement resolution limit). The fact that the Golitsyn spectrum extends at least to these scales is an indirect confirmation that turbulence is established [130], with inertial-range motions tangling the magnetic field much faster than the motions at the energy-conserving scale $\left(L_{e}\right)$. At scales of order $\ell_{e}$, the corresponding timescale is $\lesssim 25 \mathrm{~ns}$ in the laboratory, translating to $\lesssim 0.03$ Gyr in the intracluster medium over distances $\sim 1 \mathrm{Mpc}$. 

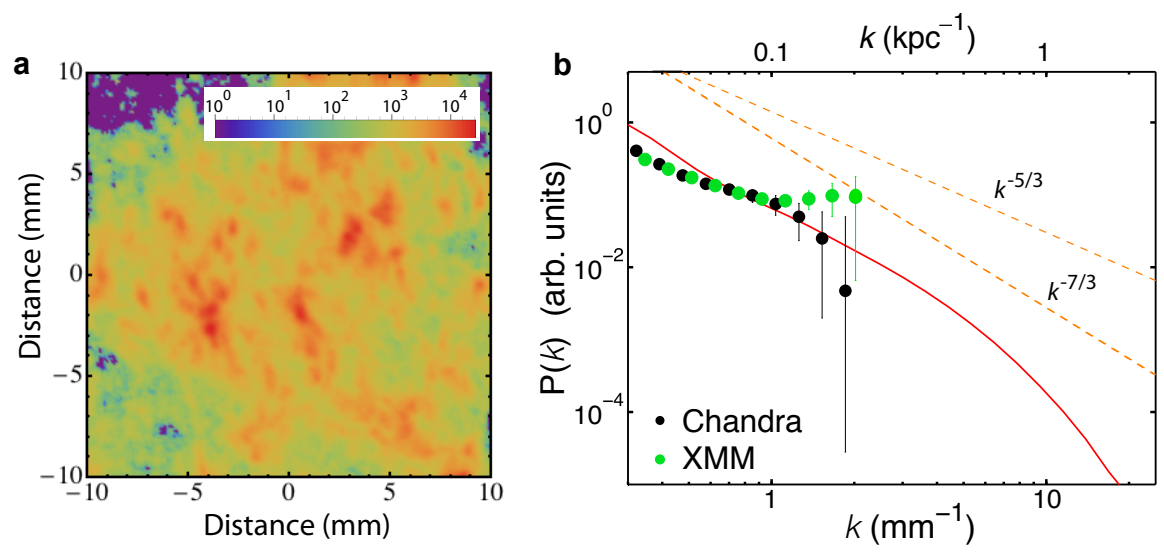

Figure 20: (a) Enlarged Schlieren image of jet collision at $\mathrm{t}=1.5 \mu \mathrm{s}$ (see Ref. [142]). (b) Calculated power spectrum from experimental data (solid line). Black and green symbols correspond to the inferred density spectrum in the Coma cluster obtained from CHANDRA and XMM satellite observations, respectively [27]. The power spectrum is arbitrarily normalized such that $P(k) \approx 1$ at the largest scale. Adapted from Ref. [142].

\section{Characterization of turbulence in the intergalactic medium and in the laboratory experiments}

During hierarchical structure formation, clusters form from accretion of filaments, galaxies, galaxy groups, and cluster mergers. These events lead to complex flows and the turbulent pressure is expected to be a few percent of the thermal pressure. In clusters of galaxies, turbulent velocities are inferred from the density perturbations, which, in turn, are obtained using the measured X-ray radiation intensities [26, 27, 143]. However, the relation between density and velocity perturbations is not entirely clear. If pressure fluctuations are the source of density perturbations and the velocity spectrum is Kolmogorov, then we would expect for the density fluctuations to exhibit a $k^{-7 / 3}$ power law [26]. To see this, we first notice that for an inviscid fluid (neglecting heat conduction and radiation transport) $d p / p=\gamma_{a} d \rho / \rho$, where $\gamma_{a}$ is the specific heat ratio. Hence pressure and density fluctuations are proportional. Also, in the Kolmogorov approximation $u_{\ell} \sim(\epsilon \ell)^{1 / 3}$, then the kinetic energy of a fluid element at the scale $\ell$ goes as $E(\ell) \sim u_{\ell}^{2}=\epsilon^{2 / 3} \ell^{2 / 3}$. The kinetic energy spectrum is thus

$$
E(k) \equiv\left|\mathbf{u}_{\mathbf{k}}\right|^{2}=\int E(\ell) e^{-i k \ell} d \ell \sim E(\ell) \ell \sim \epsilon^{2 / 3} k^{-5 / 3},
$$

where $k \sim 1 / \ell$. In the spirit of the Boussinesq approximation, since the buoyancy term dominates over the flow gradient term (i.e., the Richardson number is large), then density variations can be neglected in the fluid equations except those due to the gravity. Hence, assuming pressure is dominated by the ram term (i.e., the turbulence is supersonic), its fluctuations on a scale $\ell$ are $p_{\ell} \sim \rho u_{\ell}^{2}$, where $\rho$ is the average density in the fluid. The corresponding spectrum is thus $E_{P}(k) \sim p_{\ell}^{2} \ell \sim \epsilon^{4 / 3} \ell^{7 / 3} \sim \epsilon^{4 / 3} k^{-7 / 3}$, as we indicated above.

Conversely, if the turbulence in clusters is mainly sub-sonic at small scales (and near sonic at large scales), so density fluctuations (injected at large scales) behave like a passive scalar. This means that sounds waves are the source of density variations, thus $d \rho / \rho \sim u / c_{s}$, where $c_{s}$ is the sound speed. Therefore the density and velocity spectra are expected to be the same [144]. The situation may be more complicated when the magnetic field becomes dynamically important, as it must, due to dynamo at large $\mathrm{Rm}$. These considerations are part of a larger, fundamental question of how the energy injected at large scales into a compressible plasma is partitioned, in a turbulent cascade, between small-scale motions, magnetic and compressive fluctuations.

Results from a recent laboratory experiment aimed at the characterization of turbulent flows are shown in Figure 20. These are based on the same platform discussed above, with the difference that the turbulent flow results from the collision of two plasma jets. This is reminiscent of cluster merger events with the attempt to produce a laboratory-scale replica of a turbulent intracluster plasma, although obviously the plasma is not confined in a dark matter potential well, as it is in clusters. In the intracluster medium, large-scale turbulent motions are influenced by density stratification and gravity; however, at smaller spatial scales the time periods for buoyancy-driven motions are much longer than those 
of the turbulent motions. Therefore the fluctuations at these scales are universal, and thus similar to the turbulence we can create in our laboratory experiments. The scale invariance of hydrodynamic equations $[6,11]$ implies, if we assume that a distance of $1 \mathrm{~cm}$ in the laboratory corresponds to $100 \mathrm{kpc}$ in the astrophysical case, that $1 \mu \mathrm{s}$ becomes $0.5 \mathrm{Gyr}$ and a density of $4 \times 10^{17} \mathrm{~cm}^{-3}$ is equivalent to $0.01 \mathrm{~cm}^{-3}$ in the galaxy cluster.

The experiment was conducted using the Vulcan laser of the Central Laser Facility at the Rutherford Appleton Laboratory. Multiple laser beams (with $\sim 240 \mathrm{~J}$ total energy and $\sim 1 \mathrm{~ns}$ pulse duration) were focussed onto a carbon foil in order to launch a plasma jet into an ambient argon gas-filled chamber (at a pressure of 1 mbar). A full description of the experimental set-up is given by [142]. Ablation of target material by the laser drives a shock into the carbon foil, which then produces a collimated jet from the back surface of the target (i.e., the side opposite to that illuminated by the laser). The target material ablated by the laser is slowed by the ambient medium, creating a wrap-around shock. Schlieren measurements were taken to characterize the outflows at various times. The fastest moving material occurs on axis with $v_{0} \sim 25 \mathrm{~km} / \mathrm{s}\left(v_{0} / c_{s} \sim 4\right.$, where $c_{s}$ is the sound speed) at $3 \mathrm{~cm}$ from the target, while material on the edges of the jet moves more slowly as a result of Kelvin-Helmholtz shearing instabilities. Experiments were performed using two sets of laser beams, each set illuminating a carbon foil, producing two jets that collide. The collision drives strong turbulence in a region that grows from a size $L \sim 1 \mathrm{~cm}$ at $t=0.8 \mu \mathrm{s}$ to $\sim 2 \mathrm{~cm}$ at $t>1.5 \mu \mathrm{s}$, at which time the turbulence reaches a more relaxed state.

The measurement of the density-fluctuation spectra were performed from a Schlieren image of the turbulent region in at the collision between the two jets (see Fig. 20). In Schlieren imaging, the measured signal intensity is proportional to $\int\left(\partial n_{e} / \partial y+\partial n_{e} / \partial z\right) d x$, where $n_{e}$ is the electron density, $x, y$ are the image-plane spatial co-ordinates and $z$ the depth [94]. Therefore, under the assumption that turbulence is statistically homogeneous across the jet interaction region, the discrete Fourier transform of the central region of the jet collision directly gives the power spectrum of the density fluctuations. The result is shown in Fig. 20, using the wavelet method discussed in Ref. [27], which was used for the analysis of X-ray maps of the Coma cluster. The spectrum is consistent with either a $k^{-5 / 3}$ or a $k^{-7 / 3}$ power law, similar to what was obtained in galaxy clusters $[26,27]$. These experiments do indeed produce a turbulent plasma, somewhat similar to galaxy clusters.

Taking the measured values of jet velocity, density, and temperature in the collision region, the Reynolds number calculated with respect to the scale $L$ is $\operatorname{Re}=v_{0} L / v \sim 3 \times 10^{6}$. On the other hand the magnetic Reynolds number is $\mathrm{Rm}=v_{0} L / \eta \sim 10$, so the resistive scale lies well above the viscous scale, and close to the system scale, $L$. Since $\mathrm{Rm}$ is not very large in the experiment, the full magneto-hydrodynamic (MHD) scaling between the cluster and the laboratory is only marginally valid [11]. At such Rm, turbulent dynamo, believed to be the mechanism whereby strong fields are generated in galaxy clusters [145, 146], does not operate (see below), but the magnetic fields can be amplified via stochastic tangling of an imposed field by turbulent motions - the turbulent induction mechanisms discussed previously.

\section{The dynamo equation}

The discussion so far was that, with sufficiently large magnetic Reynolds numbers, the magnetic field dynamics in the laboratory and astrophysical systems are equivalent. Consequently, the measured magnetic field in the laboratory can be rescaled to make predictions of the value expected in a galaxy cluster which can be compared with observations. In the previous section, however, we have pointed out that the growth rate of the magnetic field at the resistive scale goes as $\gamma \sim u_{e} R m^{1 / 2} / L_{e}$, Eq. (40). This would give rise, in general, of an unequal evolution of the magnetic field in the laboratory and galaxy cluster systems because of the enormous difference in the actual values of $R m$, even if large in both cases. The difference is clearly related to the non-linear, multi-scale nature of MHD turbulence, and the dynamo process. In the presence of a turbulent fluid, we can think of the magnetic field as given by the sum of two independent terms: one is the mean (smooth and coherent) part and the other one is a random, rapidly fluctuating term. Similarly, the velocity can also be split into a smooth and turbulent term. We write:

$$
\begin{gathered}
\mathbf{B} \rightarrow \mathbf{B}+\delta \mathbf{B}, \\
\mathbf{u} \rightarrow \mathbf{u}+\delta \mathbf{u},
\end{gathered}
$$

where we assume that $\delta \mathbf{B}$ and $\delta \mathbf{u}$ average to zero over a time much longer than $\tau_{c}$ (the de-correlation time). That is, $\langle\mathbf{B}+\delta \mathbf{B}\rangle=\mathbf{B}$ and $\langle\mathbf{u}+\delta \mathbf{u}\rangle=\mathbf{u}$. Substituting the above into the induction equation (4) and taking a time average, we 
obtain

$$
\frac{\partial \mathbf{B}}{\partial t}=\nabla \times(\mathbf{u} \times \mathbf{B})+\nabla \times\langle\delta \mathbf{u} \times \delta \mathbf{B}\rangle+\eta \nabla^{2} \mathbf{B} .
$$

The main difference between this equation and the induction equation is that now $\mathbf{B}$ and $\mathbf{u}$ represent mean field values. We also see the appearance of a mean electromotive force associated with the fluctuating components $\langle\delta \mathbf{u} \times \delta \mathbf{B}\rangle$. The corresponding term in the induction equation is of central importance[15]. In practice, this is the equation we need to use when comparing with experimental data and observations, since it is the mean field that is measured, as the magnetic field which is measured by the induction probes is averaged over a finite volume (i.e., the size of the probe). The ergodic hypothesis ensures that time averages are roughly equivalent to spatial averages. The fluctuating electromotive term should in principle be solved self-consistently and re-expressed in terms of the mean components $\mathbf{B}$ and $\mathbf{u}$, such that equation 48 can be integrated. Adopting the same approximation given in [15]

$$
\langle\delta \mathbf{u} \times \delta \mathbf{B}\rangle=\alpha \mathbf{B}-\beta \nabla \times \mathbf{B},
$$

where

$$
\begin{gathered}
\alpha=-\frac{\tau_{c}}{3}\langle(\mathbf{u}+\delta \mathbf{u}) \cdot \nabla \times(\mathbf{u}+\delta \mathbf{u})\rangle \approx-\frac{\tau_{c}}{3} \mathbf{u} \cdot \nabla \times \mathbf{u}, \\
\beta=\frac{\tau_{c}}{2}\left\langle(\mathbf{u}+\delta \mathbf{u})^{2}\right\rangle \approx \frac{\tau_{c}}{3} u^{2} .
\end{gathered}
$$

we see we have the desired result. Here, the parameter $\alpha$ is related to the degree of vortex lines linkage (the helicity). For an ideal fluid (i.e., large $R e$ ) the helicity is a conserved quantity. In galaxy clusters and in the experiment discussed here turbulence is created without any injection of helicity, hence the system evolves keeping $\alpha \sim 0$. This, together with the assumption that the mean field obeys $\nabla \cdot \mathbf{B}=0$, implies the following equation for the mean field

$$
\frac{\partial \mathbf{B}}{\partial t}=\nabla \times(\mathbf{u} \times \mathbf{B})+(\beta+\eta) \nabla^{2} \mathbf{B},
$$

thus $\beta$ essentially behaves as turbulent resistivity, effectively increasing or decreasing electrical currents in a nontrivial manner. Indeed experiment in liquid metals have shown that $\beta$ can take both positive and negative values at different scales [147]. In order to get a better understanding of the induction equation and its relevance in the context of previous (and future) laser-based plasma experiments, let's consider its dimensionless form, obtained following the same procedure as discussed in Section 4. We have

$$
\frac{\partial \mathbf{B}^{*}}{\partial t^{*}}=\nabla^{*} \times\left(\mathbf{u}^{*} \times \mathbf{B}^{*}\right)+\frac{1}{D y} \nabla^{2 *} \mathbf{B}^{*}+\frac{1}{R m} \nabla^{2 *} \mathbf{B}^{*},
$$

where the a new non-dimensional number has appeared:

$$
D y=\frac{u_{0} \ell_{0}}{\beta},
$$

with $\ell_{0}$ and $u_{0}$ the characteristic spatial and velocity scales. Taking those scales to be the ones associated to the outer motions, and, for typical experimental conditions where $R e \gg R m$, the de-correlation time corresponds to the eddy-turnover time on the scale set by the probe size. Since this scale is close to the dissipation scale, $\tau_{c} \sim 1 / \gamma=$ $\ell_{0} R m^{-1 / 2} / u_{0}$. Also, we can approximate the turbulent resistivity as [15] $\beta=\tau_{c} u_{0}^{2} / 3$, thus $D y \sim 3 R m^{-1 / 2}$ and the dimensionless mean field dynamo equation equation reads as

$$
\frac{\partial \mathbf{B}^{*}}{\partial t^{*}}=\nabla^{*} \times\left(\mathbf{u}^{*} \times \mathbf{B}^{*}\right)+\frac{3}{R m^{1 / 2}} \nabla^{2 *} \mathbf{B}^{*}+\frac{1}{R m} \nabla^{2 *} \mathbf{B}^{*} .
$$

This result is very instructive. The growth of the magnetic field is driven by the induction term $\nabla^{*} \times\left(\mathbf{u}^{*} \times \mathbf{B}^{*}\right)$. For dynamo to be operative, we want this term to be much larger than the diffusion ones, say at least ten times larger. While a magnetic Reynolds number $R m \sim 10$ is sufficient to make the regular resistive term negligible, turbulent diffusion still remains substantial, due to the much weaker dependence with $R m$. This implies that $R m$ must be at least a few hundreds in order for $3 / \mathrm{Rm}^{1 / 2}$ to be small enough. Indeed, numerical simulations indicate that the critical $R m$ for the onset of dynamo is near or above 100 [130]. 


\section{Further applications - particle acceleration}

The main focus of this review has been to summarise experimental efforts to study magnetogenisis and field amplification in laboratory plasmas, and their relevance to field generation in the Universe. It is clear that shocks play a major role in this study. Astrophysical shocks are typically associated with significant plasma heating, as well as non-thermal particle acceleration. This is found to hold at almost all scales measured, from coronal mass ejection (CME) shocks propagating in the stellar wind [148], to radio relics [149], produced in the merger of galaxies. The theory of diffusive shock acceleration $[58,55,57,56]$, sometimes called first-order Fermi acceleration ${ }^{4}$ has proved to be remarkably successful in accounting for observation of non-thermal particle signatures at shocks. It is also argued that this process, occurring at supernova remnant shocks, is the primary source of cosmic rays in our Galaxy $[151,152,153]$. The origin of cosmic rays is now a century old problem. Originally discover in 1912 in a series of balloon experiments [154], today, through a combination of balloon, satellite and ground based experiments, we have a wealth of data, providing us with detailed measurements of both the spectral energy distribution and composition of cosmic rays. Recent gamma-ray measurements from the Fermi satellite have also revealed the presence of the long-sought after pion-decay signature, resulting from the decay of $\pi^{0}$ mesons produced in the collisions between accelerated and thermal protons [155]. Hence the evidence is mounting that supernovae are indeed the sources of Galactic cosmic rays.

However, the theory is not complete. The two major obstacles are the injection mechanism; how particles are lifted from the thermal pool into the diffusive process; and the maximum energy. While numerical simulations are advancing our understanding of these processes $[156,157,158]$, combining the two is extremely difficult, since a fully self-consistent model would need to accurately simulate the physics from scales relevant to the thermal particles, up the maybe $\mathrm{PeV}$ energies. This is currently intractable, for numerous reasons.

The question arises, as to where laser produced shock experiments can provide input into this field. While kinetic simulations of astrophysical shocks seem to imply that particle acceleration of some description, is a natural outcome of collisionless shock formation, recent work has demonstrated that provided the shock heated electrons are sufficiently magnetised, i.e. $\omega_{g} \tau>1$, super-thermal particles can still participate in the diffusive shock acceleration mechanism [159]. The primary experimental difficulty for diffusive shock acceleration measurements in the laboratory, concerns distinguishing accelerated particles from shock heated particles. Clearly the larger the separation between these two populations the better. Generally speaking, an upper limit to the maximum energy that a particle can accelerated to in any system, is given by the so-called Hillas criterion, which for a non-relativistic particle reads

$$
\varepsilon_{\max }<Z e \int \boldsymbol{E} \cdot d \boldsymbol{r}<5 Z\left(\frac{u}{10^{6} \mathrm{~ms}^{-1}}\right)\left(\frac{B}{1 \mathrm{~T}}\right)\left(\frac{R}{1 \mathrm{~cm}}\right) \mathrm{keV},
$$

where $Z$ is the charge, $R$ the system size and $u$ the characteristic velocity of scatterers, here corresponding to the shock velocity. We have also assumed the electric field is well approximated by $\boldsymbol{E}=-\boldsymbol{u} \times \boldsymbol{B}$. Comparing this with the standard Rankine-Hugoniot conditions for a strong (sonic Mach number $M \gg 1$ ) hydrodynamic shock in gas with adiabatic index $\gamma=5 / 3$,

$$
k_{\mathrm{B}} T_{\mathrm{i}}=\frac{3}{16} m_{i} u_{\mathrm{sh}}^{2}=2 \mu_{i}\left(\frac{u}{10^{6} \mathrm{~ms}^{-1}}\right)^{2} \mathrm{keV},
$$

where $\mu_{i}=m_{i} / m_{p}$, with $m_{p}$ the proton mass. Electron heating will depend on both the nature of the shock, but is typically larger than $\left(m_{e} / m_{i}\right) T_{i}$ and smaller than $T_{i}$. Results from collisionless shock simulations suggest $T_{e} \sim 0.1 T_{i}$, which is reasonably well supported by in situ measurements of interplanetary shocks, and supernova observations [160], with a general trend of decreasing temperature ration with increasing Alfvénic Mach number.

The shock velocity used in these calculations is clearly larger than that measured in the experiments discussed in sections 9 and 13. However, those shocks were highly collisional, to the extent that electrons were completely unmagnetised. As we will demonstrate, the laser energy of at least a kiloJoule is required to carry out the investigations we envisage. Nevertheless, we could consider a similar experimental set-up, whereby a central target is irradiated,

\footnotetext{
${ }^{4}$ As opposed to the original acceleration mechanism proposed by Fermi [150] where particles were elastically scattered off random distribution of slowly moving clouds. The average fractional energy change per collision in this case is $\Delta E / E \propto<u^{2}>/ \mathrm{v}^{2}$, where $<u^{2}>$ is the mean square of the cloud velocities, and $v$ the speed of the accelerating particle. Modern theories have replaced the clouds by MHD waves, and typically refer to any mechanism of this type as second order Fermi acceleration.
} 
launching a spherical shock through a gas filled chamber. Assuming a efficiency factor $\eta=0.01$ for conversion of laser energy into the blast wave, the Sedov-Taylor blast wave solution is

$$
u_{\mathrm{sh}} \approx 10^{6} \mu_{i}^{-1 / 2}\left(\frac{\eta}{0.01}\right)^{1 / 2}\left(\frac{E}{1 \mathrm{~kJ}}\right)^{1 / 2}\left(\frac{n_{\mathrm{ext}}}{10^{16} \mathrm{~cm}^{-3}}\right)^{-1 / 2}\left(\frac{R}{1 \mathrm{~cm}}\right)^{-3 / 2} \mathrm{~m} \mathrm{~s}^{-1}
$$

where $n_{\text {ext }}$ is the number density of ambient gas in the chamber and $\mu_{i}$ its mean atomic mass. Thus, our fiducial shock velocity is not unreasonable, and comparing Equations (56) and (57) it is immediately apparent that distinguishing thermal and non-thermal ions presents a serious challenge. Hence we focus on the possibility of detecting non-thermal electrons.

As already stated, diffusive shock acceleration requires the background electrons to be magnetised. From the electron Coulomb scattering time $\tau_{e}$ [49], we find

$$
\omega_{g} \tau_{e} \approx 0.6\left(\frac{B}{1 \mathrm{~T}}\right)\left(\frac{n}{10^{16} \mathrm{~cm}^{-3}}\right)^{-1}\left(\frac{\varepsilon_{e}}{1 \mathrm{eV}}\right)^{3 / 2}
$$

Self-generated fields produced at the shock will generally be insufficient to achieve $\omega_{g} \tau_{e}>1$, although with an external 1 Tesla magnet, modest shock heating should be enough to ensure the electrons are magnetised. The use of an external magnetic field is also vital to confine the particles to the shock, facilitating multiple crossing, as required to accelerate up to the maximum energy given in Equation (56). If, for example, one relies exclusively on Biermann battery fields generated downstream of the shock, any electrons that venture upstream of the shock will simply outrun it. While the flux of electrons may excite various plasma instabilities, the time taken to isotropise these streaming particles is not sufficient to allow any appreciable acceleration. In fact, a necessary condition for acceleration in the laboratory is that the acceleration time is shorter than the Coulomb collision time:

$$
t_{\mathrm{acc}} \sim \frac{p}{\langle\Delta p\rangle} t_{\mathrm{c}} \approx \frac{\mathrm{v} t_{\mathrm{c}}}{u_{\mathrm{sh}}}<\tau_{e}
$$

where $\Delta p$ is the average kinematic change in particle momentum on crossing the shock ${ }^{5}$, and $t_{\mathrm{c}}$ is the average time taken to perform one shock crossing cycle. This time will depend on the orientation of the mean magnetic field, and how effectively small fluctuations in the field can scatter non-thermal particles. Defining a collisionless scattering time, $\tau_{B}$ similar to the Coulomb scattering time, if the shock normal is in the direction $\boldsymbol{n}$, provided $\omega_{g} \tau_{B}>1$, which is expected in most circumstances, we can approximate $t_{\mathrm{c}}$ in the two limiting field orientations $[161,153,159]$

$$
\omega_{g} t_{\mathrm{c}} \approx \frac{\mathrm{V}}{u_{\mathrm{sh}}}\left\{\begin{array}{cc}
\omega_{g} \tau_{B} & \boldsymbol{B} \| \boldsymbol{n} \\
\left(\omega_{g} \tau_{B}\right)^{-1} & \boldsymbol{B} \perp \boldsymbol{n}
\end{array}\right.
$$

Clearly acceleration can operate faster at perpendicular shocks with strong fields. We thus acceleration at perpendicular shocks, since if it is only marginally possible at these shocks, it will be impossible at parallel shocks. If we take the optimal conditions for acceleration, i.e. $\omega_{g} \tau_{B}=\mathrm{v} / u_{\mathrm{sh}}[162,163]$, such that $\omega_{g} t_{\mathrm{c}} \approx 1$, the inequality in equation (60) can be used to find a minimum energy for acceleration to dominate over Coulomb losses

$$
\frac{\varepsilon_{\min }}{1 \mathrm{eV}}>\operatorname{Max}\left[\frac{20}{\mu_{i}}\left(\frac{\eta}{0.01}\right)\left(\frac{E}{1 \mathrm{~kJ}}\right)\left(\frac{n_{\mathrm{ext}}}{10^{16} \mathrm{~cm}^{-3}}\right)^{-1}\left(\frac{R}{1 \mathrm{~cm}}\right)^{-3}, 25 \mu_{i}^{2}\left(\frac{\eta}{0.01}\right)^{-2}\left(\frac{E}{1 \mathrm{~kJ}}\right)^{-2}\left(\frac{B}{1 \mathrm{~T}}\right)^{-2}\left(\frac{R}{1 \mathrm{~cm}}\right)^{6}\right]
$$

Here, we have also taken into account that the minimum energy that satisfies the collsionless condition, might not necessarily be sufficiently fast to outrun the shock. The first entry in the minimum function, thus corresponds to an electron with energy $0.5 m_{e} u_{\mathrm{sh}}^{2}$.

\footnotetext{
${ }^{5}$ If, as is commonly done in astrophysical systems, the momentum is measured in the local fluid frame, where in the ideal MHD approximation, the local electric field vanishes, this momentum change is purely due to the change in reference frames between the converging flows. It is trivial to show that at a shock front, this momentum change is always positive, both for upstream and downstream transitions, in both cases $<\Delta p>/ p \sim u_{\mathrm{sh}} / \mathrm{v}$. It is this scaling that has resulted in the acceleration mechanism being frequently referred to as a first order Fermi mechanism, compared with the original acceleration mechanism proposed by Fermi, which resulted in a slower momentum space diffusion that was a second order process.
} 
While it might appear the larger the magnetic field the better, it is important that the magnetic pressure upstream does not exceed the ram pressure of the shock. This is equivalent to requiring the shock remains super-Alfvénic

$$
M_{\mathrm{A}} \approx 5\left(\frac{\eta}{0.01}\right)^{1 / 2}\left(\frac{E}{1 \mathrm{~kJ}}\right)^{1 / 2}\left(\frac{B}{1 \mathrm{~T}}\right)^{-1}\left(\frac{R}{1 \mathrm{~cm}}\right)^{-3 / 2}>1
$$

We thus have three conditions that must be fulfilled to successfully and unambiguously detect electron acceleration in the laboratory: 1) clear separation between thermal and maximum energy particles; 2) acceleration faster than Coulomb collision rate; 3) super-Alfvenic shock. From these three constraints, we can construct a parameter space to locate regions where a clear detection of particle acceleration can be made.

The first condition (separation between thermal and non-thermal particles by at least a factor of 10) requires

$$
R_{\mathrm{sh}}>0.5 \mu_{i}^{-1 / 5}\left(\frac{\eta}{0.01}\right)^{1 / 5}\left(\frac{E}{1 \mathrm{~kJ}}\right)^{1 / 5}\left(\frac{B}{1 \mathrm{~T}}\right)^{-2 / 5}\left(\frac{n_{\mathrm{ext}}}{10^{16} \mathrm{~cm}^{-3}}\right)^{-1 / 5} \mathrm{~cm}
$$

while the latter two conditions require

$$
R_{\mathrm{sh}}<\operatorname{Min}\left[2 \mu_{i}^{-1 / 2}\left(\frac{\eta}{0.01}\right)^{1 / 3}\left(\frac{E}{1 \mathrm{~kJ}}\right)^{1 / 3}\left(\frac{B}{1 \mathrm{~T}}\right)^{2 / 9}\left(\frac{n_{\mathrm{ext}}}{10^{16} \mathrm{~cm}^{-3}}\right)^{-5 / 9},\left(\frac{\eta}{0.01}\right)^{1 / 3}\left(\frac{E}{1 \mathrm{~kJ}}\right)^{1 / 3}\left(\frac{B}{1 \mathrm{~T}}\right)^{-2 / 3}\right] \mathrm{cm},
$$

where for the latter limit, we chose for convenience $M_{\mathrm{A}}>5$.

From these scalings, we can see a number of obvious effects. Firstly, the window for successful detection is quite narrow for the given parameters. Moving from a kiloJoule class laser to a MegaJoule laser presents only a small advantage. Using lower background densities also offers a slight improvement, but makes other diagnostics, such as interferometry, a challenge. However, a successful detection should be achievable in a carefully designed experiment, with controls.

\section{Conclusions and Outlook}

Many of the problems faced by astrophysicists working in the field of galaxy clusters are fundamental plasma physics problems. In this review we have attempted to motivate how carefully designed laser-based experiments can provide further insight into the inner workings of these giant structures, and specifically provide important clues regarding the mechanisms involved in the generation and amplification of magnetic fields. Magnetic field generation by the Biermann battery mechanism, the Weibel instability and resistive return currents have been demonstrated in several laboratory experiments for conditions relevant to galaxy clusters. The experiments are used as an important benchmarking tool for simulations codes, particularly when the time and spatial scales are well beyond the linear growth regimes. Laboratory experiments can now achieve very large fluid Reynolds numbers, in excess of what currently achievable in numerical simulations. This implies that the hydrodynamic equations correctly scale between the laboratory and galaxy cluster, reproducing all the relevant features of turbulent flows at scales smaller than the buoyancy scale due to density stratification. Turbulence is believed to be the main driver mechanism for the amplification of magnetic fields. At small magnetic Reynolds numbers, the amplification is set by the balance between stochastic tangling of the field lines and Ohmic diffusion. This has been seen in numerical simulations and recently applied to laboratory experiments. Future experiments on large laser facilities, such as the National Ignition Facility, could also achieve large magnetic Reynolds number, above the dynamo threshold and thus answer fundamental questions such as the saturation of the magnetic field by the back-reaction of the fluid and the time needed to reach saturation. These new experiments may even be able to access the weakly collisional magnetised regime in which the ion Larmor radius falls below the collisional mean free path. Moreover, experiments could also reach the regime where radiative losses become dynamically important to change the structure of the turbulence. How magnetic field evolves in this radiative and weakly collisional MHD regime is an area of active current research in plasma physics, and where laboratory experimental studies are likely to be ahead of observations and numerical simulations. This is an exciting time where novel discoveries are ahead of us. 


\section{Acknowledgements}

The research leading to these results has received funding from the European Research Council under the European Community's Seventh Framework Programme (FP7/2007-2013) / ERC grant agreement no. 256973. Partial support from the Science and Technology Facilities Council and the Engineering and Physical Sciences Research Council of the United Kingdom is also acknowledged.

[1] B. A. Remington, D. Arnett, R. P. Drake, H. Takabe, Modeling Astrophysical Phenomena in the Laboratory with Intense Lasers, Science 284 (1999) 1488-1493.

[2] R. Drake, High-Energy-Density Physics: Fundamentals, Inertial Fusion, and Experimental Astrophysics, Shock Wave and High Pressure Phenomena, Springer, 2006.

[3] B. A. Remington, R. P. Drake, D. D. Ryutov, Experimental astrophysics with high power lasers and z pinches, Rev. Mod. Phys. 78 (2006) 755.

[4] D. W. Savin, N. S. Brickhouse, J. J. Cowan, R. P. Drake, S. R. Federman, G. J. Ferland, A. Frank, M. S. Gudipati, W. C. Haxton, E. Herbst, S. Profumo, F. Salama, L. M. Ziurys, E. G. Zweibel, The impact of recent advances in laboratory astrophysics on our understanding of the cosmos, Reports on Progress in Physics 75 (2012) 036901.

[5] J. A. Harte, Lasnex: A 2-d physics code for modeling icd, LLNL Quarterly Report 6 (1996) UCRL-LR-105821-96-4.

[6] D. Ryutov, R. P. Drake, J. Kane, E. Liang, B. A. Remington, W. M. Wood-Vasey, Similarity Criteria for the Laboratory Simulation of Supernova Hydrodynamics, Astrophys. J. 518 (1999) 821-832.

[7] D. Ryutov, R. P. Drake, B. A. Remington, Criteria for scaled laboratory simulations of astrophysical MHD phenomena, The Astrophysical Journal 465 (2) (2000) 465-468.

[8] D. D. Ryutov, B. a. Remington, H. F. Robey, R. P. Drake, Magnetohydrodynamic scaling: From astrophysics to the laboratory, Physics of Plasmas 8 (5) (2001) 1804

[9] E. Falize, B. Loupias, A. Ravasio, C. D. Gregory, A. Dizière, M. Koenig, C. Michaut, C. Cavet, P. Barroso, J.-P. Leidinger, X. Ribeyre, J. Breil, H. Takabe, Y. Sakawa, Y. Kuramitsu, T. Morita, N. C. Woolsey, W. Nazarov, S. Pikuz, The scalability of the accretion column in magnetic cataclysmic variables: the POLAR project, Astrophysics and Space Science 336 (2011) 81-85.

[10] E. Falize, C. Michaut, S. Bouquet, Similarity Properties and Scaling Laws of Radiation Hydrodynamic Flows in Laboratory Astrophysics, The Astrophysical Journal 730 (2011) 96.

[11] J. E. Cross, B. Reville, G. Gregori, Scaling of magneto-quantum-radiative hydrodynamic equations: From laser-produced plasmas to astrophysics, Astrophys. J. 795 (2014) 59.

[12] J. Connor, J. Taylor, Scaling laws for plasma confinement, Nuclear Fusion 15 (1977) 1047.

[13] D. D. Ryutov, N. L. Kugland, H. S. Park, C. Plechaty, B. a. Remington, J. S. Ross, Basic scalings for collisionless-shock experiments in a plasma without pre-imposed magnetic field, Plasma Physics and Controlled Fusion 54 (10) (2012) 105021.

[14] I. B. Zeldovich, A. A. Ruzmaikin, D. D. Sokolov, Magnetic fields in astrophysics, Gordon \& Breach, New York, 1990.

[15] E. G. Zweibel, R. M. Kulsrud, On the origin of cosmic magnetic fields, Reports On Progress In Physics 71 (2008) 046901.

[16] P. P. Kronberg, Extragalactic magnetic fields., Rep. Prog. Phys. (1994) 325-382.

[17] C. L. Carilli, G. B. Taylor, Cluster Magnetic Fields, Annual Review of Astronomy and Astrophysics 40 (2002) 319

[18] F. Govoni, L. Feretti, Magnetic fields in clusters of galaxies, Int J Mod Phys D 13 (2004) 1549-1594.

[19] A. Neronov, I. Vovk, Evidence for strong extragalactic magnetic fields from Fermi observations of TeV blazars., Science 328 (5974) (2010) 73-75.

[20] F. Tavecchio, G. Ghisellini, L. Foschini, G. Bonnoli, G. Ghirlanda, P. Coppi, The intergalactic magnetic field constrained by Fermi/Large Area Telescope observations of the TeV blazar 1ES0229+200, Monthly Notices of the Royal Astronomical Society: Letters 406 (1) (2010) L70-L74.

[21] F. Tavecchio, G. Ghisellini, G. Bonnoli, L. Foschini, Extreme TeV blazars and the intergalactic magnetic field, Monthly Notices of the Royal Astronomical Society 414 (4) (2011) 3566-3576.

[22] E. N. Parker, Hydromagnetic Dynamo Models., ApJ 122 (1955) 293.

[23] A. Brandenburg, K. Subramanian, Astrophysical magnetic fields and nonlinear dynamo theory, Physics Reports 417 (2005) 1-209.

[24] S. I. Vaŭnshteǔn, Y. B. Zel'dovich, REVIEWS OF TOPICAL PROBLEMS: Origin of Magnetic Fields in Astrophysics (Turbulent "Dynamo" Mechanisms), Soviet Physics Uspekhi 15 (2) (1972) 159-172.

[25] F. Miniati, D. Ryu, H. Kang, T. W. Jones, R. Cen, J. P. Ostriker, Properties of cosmic shock waves in large-scale structure formation., Astrophys. J. 542 (2000) 608-621.

[26] P. Schuecker, A. Finoguenov, F. Miniati, H. Böhringer, U. Briel, Probing turbulence in the Coma galaxy cluster, Astron. Astrophys. 426 (2004) 387-397.

[27] E. Churazov, A. Vikhlinin, I. Zhuravleva, A. Schekochihin, I. Parrish, R. Sunyaev, W. Forman, H. Böhringer, S. Randall, X-ray surface brightness and gas density fluctuations in the Coma cluster, Mon. Not. R. Astron. Soc. 421 (2012) 1123-1135.

[28] F. Miniati, The Matryoshka Run: A Eulerian Refinement Strategy to Study the Statistics of Turbulence in Virialized Cosmic Structures, The Astrophysical Journal 782 (1) (2014) 21.

[29] F. Miniati, The Matryoshka Run (II): Time Dependent Turbulence Statistics, Stochastic Particle Acceleration and Microphysics Impact in a Massive Galaxy Cluster, arXiv.org (2014) 3576arXiv:1409.3576.

[30] F. Vazza, G. Brunetti, C. Gheller, R. Brunino, M. Brüggen, Massive and refined. II. The statistical properties of turbulent motions in massive galaxy clusters with high spatial resolution, Astronomy and Astrophysics 529 (2011) 17.

[31] V. L. Ginzburg, Theoretical physics and astrophysics (3rd revised and enlarged edition), Pergamon, 1979.

[32] E. N. Parker, Dynamical Instability in an Anisotropic Ionized Gas of Low Density, Physical Review 109 (6) (1958) 1874-1876.

[33] A. A. Schekochihin, S. C. Cowley, R. M. Kulsrud, M. S. Rosin, T. Heinemann, Nonlinear Growth of Firehose and Mirror Fluctuations in Astrophysical Plasmas, Physical Review Letters 100 (8) (2008) 81301. 
[34] A. Schlüter, I. Biermann, Interstellare Magnetfelder, Zeitschrift Naturforschung Teil A 5 (1950) 237.

[35] A. A. Schekochihin, S. C. Cowley, Turbulence and Magnetic Fields in Astrophysical Plasmas, Magnetohydrodynamics: Historical Evolution and Trends (2007) 85.

[36] A. Beresnyak, Basic properties of magnetohydrodynamic turbulence in the inertial range, Monthly Notices of the Royal Astronomical Society 422 (4) (2012) 3495-3502.

[37] A. Beresnyak, Universal Nonlinear Small-Scale Dynamo, Physical Review Letters 108 (3) (2012) 35002.

[38] F. Miniati, A. Beresnyak, Self-similarity of dynamo action in the largest cosmic structures, Submitted.

[39] L. M. Widrow, Origin of galactic and extragalactic magnetic fields, Reviews of Modern Physics 74 (3) (2002) 775-823.

[40] L. D. Landau, E. M. Lifshitz, Fluid mechanics; 2nd ed., Course of theoretical physics, Butterworth, Oxford, 1987.

[41] R. M. Kulsrud, R. Cen, J. P. Ostriker, D. Ryu, The protogalactic origin for cosmic magnetic fields, Astrophys. J. 480 (1997) 481-491.

[42] E. S. Weibel, Spontaneously Growing Transverse Waves in a Plasma Due to an Anisotropic Velocity Distribution, Physical Review Letters 2 (3) (1959) 83-84.

[43] S. S. Moiseev, R. Z. Sagdeev, Collisionless shock waves in a plasma in a weak magnetic field, Journal of Nuclear Energy. Part C 5 (1963) 43.

[44] R. Schlickeiser, P. K. Shukla, Cosmological Magnetic Field Generation by the Weibel Instability, Astrophys. J. 599 (2003) L57-L60.

[45] M. V. Medvedev, L. O. Silva, M. Kamionkowski, Cluster Magnetic Fields from Large-Scale Structure and Galaxy Cluster Shocks, Astrophys. J. 642 (2006) L1-L4.

[46] P. Chang, A. Spitkovsky, J. Arons, Long-Term Evolution of Magnetic Turbulence in Relativistic Collisionless Shocks: Electron-Positron Plasmas, Astrophys. J. 674 (2008) 378-387.

[47] F. Miniati, A. R. Bell, Resistive Magnetic Field Generation at Cosmic Dawn, The Astrophysical Journal 729 (1) (2011) 73.

[48] F. Miniati, A. R. Bell, Resistive Generation of Intergalactic Magnetic Field at Cosmic Dawn, in: Numerical modeling of space plasma flows (astronum 2011). Proceedings of a 6th internation conference held at Velancia, 2012, p. 125.

[49] L. Spitzer, Physics of fully ionized gases, Interscience Tracts on Physics and Astronomy, New York: Interscience Publication, 1965, 2nd rev. ed., 1965.

[50] J. Stamper, B. Ripin, Faraday-Rotation measurements of megagauss magnetic fields in laser-produced plasmas, Physical Review Letters 34 (1975) 138-141.

[51] K. Subramanian, A. Shukurov, N. E. L. Haugen, Evolving turbulence and magnetic fields in galaxy clusters, Monthly Notices of the Royal Astronomical Society 366 (4) (2006) 1437-1454.

[52] N. Y. Gnedin, A. Ferrara, E. G. Zweibel, Generation of the Primordial Magnetic Fields during Cosmological Reionization, The Astrophysical Journal 539 (2) (2000) 505-516.

[53] K. Subramanian, D. Narasimha, S. Chitre, Thermal generation of cosmological seed magnetic fields in ionization fronts, Monthly Notices of the Royal Society 271 (1994) L15-L18.

[54] N. Y. Gnedin, A. Ferrara, E. G. Zweibel, Generation of the Primordial Magnetic Fields during Cosmological Reionization, Astrophysical Journal 539 (2) (2000) 505-516.

[55] G. F. Krymskii, A regular mechanism for the acceleration of charged particles on the front of a shock wave, Akademiia Nauk SSSR Doklady 234 (1977) 1306-1308.

[56] W. I. Axford, E. Leer, G. Skadron, The acceleration of cosmic rays by shock waves, International Cosmic Ray Conference 11 (1977) $132-137$.

[57] R. D. Blandford, J. P. Ostriker, Particle acceleration by astrophysical shocks, ApJL 221 (1978) L29-L32. doi:10.1086/182658.

[58] A. R. Bell, The acceleration of cosmic rays in shock fronts. I, MNRAS182 (1978) 147-156.

[59] V. S. Berezinskii, S. V. Bulanov, V. L. Ginzburg, V. A. Dogel, V. S. Ptuskin, The astrophysics of cosmic rays, Moscow.

[60] A. Bamba, R. Yamazaki, M. Ueno, K. Koyama, Studying astrophysical collisionless shocks with counterstreaming plasmas from high power lasers, Astrophys. J. 589 (2003) 827-837.

[61] H. Park, D. Ryutov, J. Ross, N. Kugland, S. Glenzer, C. Plechaty, S. Pollaine, B. Remington, a. Spitkovsky, L. Gargate, G. Gregori, a. Bell, C. Murphy, Y. Sakawa, Y. Kuramitsu, T. Morita, H. Takabe, D. Froula, G. Fiksel, F. Miniati, M. Koenig, a. Ravasio, a. Pelka, E. Liang, N. Woolsey, C. Kuranz, R. Drake, M. Grosskopf, Small-scale structure of the SN 1006 shock with chandra observations, High Energy Density Phys. 8 (2012) 38-45.

[62] R. Treumann, Fundamentals of collisionless shocks for astrophysical application, 1. non-relativistic shocks, Astron. Astrophys. Rev. 17 (2009) 409-535.

[63] C. Niemann, W. Gekelman, C. Constantin, T. Everson, D. Schaeffer, A. Bondarenko, S. Clark, D. Winske, S. Vincena, B. Van Compernolle, P. Pribyl, Observation of collisionless shocks in a large current-free laboratory plasma, Geophys. Res. Lett. 41 (2014) 7413-7418.

[64] T. Kato, H. Takabe, Nonrelativistic collisionless shocks in unmagnetized Electron-Ion plasmas, Astrophys. J. 681 (2008) L93-L96.

[65] M. Haines, Magnetic-field generation in laser fusion and hot-electron transport, Canadian Journal of Physics 64 (1986) 912.

[66] F. Shu, The Physics of Astrophysics Volume II: Gas Dynamics, University Science Books, 1992.

[67] D. Mihalas, B. Mihalas, Foundations of Radiation Hydrodynamics, Dover Books on Physics, Dover, 1999.

[68] Y. Zel'dovich, Y. Raizer, Physics of Shock Waves and High-temperature Hydrodynamic Phenomena, Dover Books on Physics Series, Dover Publications, 2002.

[69] J. Castor, Radiation Hydrodynamics, Cambridge University Press, 2004.

[70] R. G. McClarren, R. P. Drake, J. E. Morel, J. P. Holloway, Theory of radiative shocks in the mixed, optically thick-thin case, Physics of Plasmas 17 (9) (2010) 093301.

[71] F. Haas, Quantum Plasmas: An Hydrodynamic Approach, Springer Series on Atomic, Springer New York, 2011.

[72] A. Chapman, T. G. Cowling, The Mathematical Theory of Non- Uniform Gases, Cambridge University Press, 1970

[73] H. Alfvén, Existence of Electromagnetic-Hydrodynamic Waves, Nature 150 (1942) 405-406.

[74] A. G. Kritsuk, Å. Nordlund, D. Collins, P. Padoan, M. L. Norman, T. Abel, R. Banerjee, C. Federrath, M. Flock, D. Lee, P. S. Li, W.-C. Müller, R. Teyssier, S. D. Ustyugov, C. Vogel, H. Xu, Comparing Numerical Methods for Isothermal Magnetized Supersonic Turbulence, 
The Astrophysical Journal 737 (2011) 13.

[75] M. Wan, S. Oughton, S. Servidio, W. H. Matthaeus, On the accuracy of simulations of turbulence, Physics of Plasmas 17 (8) (2010) 082308.

[76] M. J. Berger, J. Oliger, Adaptive Mesh Refinement for Hyperbolic Partial Differential Equations, Journal of Computational Physics 53 (1984) 484-512.

[77] D. W. Hughes, S. A. E. G. Falle, The Rise of Twisted Magnetic Flux Tubes: A High Reynolds NumberAdaptive Grid Calculation, ApJL 509 (1998) L57-L60.

[78] B. Fryxell, K. Olson, P. Ricker, F. X. Timmes, M. Zingale, D. Q. Lamb, P. MacNeice, R. Rosner, J. W. Truran, H. Tufo, FLASH: An Adaptive Mesh Hydrodynamics Code for Modeling Astrophysical Thermonuclear Flashes, Astrophys. J. Suppl. 131 (2000) $273-334$.

[79] F. Miniati, P. Colella, Block structured adaptive mesh and time refinement for hybrid, hyperbolic + N-body systems, Journal of Computational Physics 227 (2007) 400-430.

[80] C. Graziani, P. Tzeferacos, D. Lee, D. Lamb, K. Weide, M. Fatenejad, J. Miller, The biermann catastrophe in numerical magnetohydrodynamics, Astrophys. J. 802 (2015) 43.

[81] J. L. Maron, G. G. Howes, Gradient Particle Magnetohydrodynamics: A Lagrangian Particle Code for Astrophysical Magnetohydrodynamics, The Astrophysical Journal 595 (2003) 564-572.

[82] J. Meinecke, H. W. Doyle, F. Miniati, A. R. Bell, R. Bingham, R. Crowston, R. P. Drake, M. Fatenejad, M. Koenig, Y. Kuramitsu, C. C. Kuranz, D. Q. Lamb, D. Lee, M. J. Macdonald, C. D. Murphy, H.-S. Park, A. Pelka, A. Ravasio, Y. Sakawa, A. A. Schekochihin, A. Scopatz, P. Tzeferacos, W. C. Wan, N. C. Woolsey, R. Yurchak, B. Reville, G. Gregori, Turbulent amplification of magnetic fields in laboratory laserproduced shock waves, Nature Phys. 10 (2014) 520-524.

[83] T. Boehly, R. McCrory, C. Verdon, W. Seka, S. Loucks, A. Babushkin, R. Bahr, R. Boni, D. Bradley, R. Craxton, J. Delettrez, W. Donaldson, R. Epstein, D. Harding, P. Jaanimagi, S. Jacobs, K. Kearney, R. Keck, J. Kelly, T. Kessler, R. Kremens, J. Knauer, D. Lonobile, L. Lund, F. Marshall, P. McKenty, D. Meyerhofer, S. Morse, A. Okishev, S. Papernov, G. Pien, T. Safford, J. Schnittman, R. Short, M. S. III, M. Skeldon, S. Skupsky, A. Schmid, V. Smalyuk, D. Smith, J. Soures, M. Wittman, B. Yaakobi, Inertial confinement fusion experiments with omega-a 30-kj, 60-beam \{UV\} laser, Fusion Engineering and Design 44 (1999) 35 - 42.

[84] E. I. Moses, The national ignition facility: Transition to a target shooter, in: B. Hammel, D. Meyerhofer, J. Meyher-ter Vehn, A. H. (Eds.), 3rd International Conference on Inertial Fusion Sciences and Applications, American Nuclear Society, Monterey, CA, 2003, p. 535540.

[85] A. Bettinger, M. Decroisette, Laser megajoule project and impact on the inertial fusion program, Fusion Engineering and Design 46 (1999) $457-460$.

[86] S. Glenzer, R. Redmer, X-ray thomson scattering in high energy density plasmas, Rev. Mod. Phys. 81 (4) (2009) 1625-1663.

[87] J. Lindl, Development of the indirect drive approach to inertial confinement fusion and the target physics, Phys. Plasmas 2 (1995) 39334024

[88] J. Nuckolls, L. Wood, A. Thiessen, G. Zimmerman, Laser compression of matter to Super-High densities: Thermonuclear (CTR) applications, Nature 239 (5368) (1972) 139-142.

[89] O. Hurricane, D. Callahan, D. Casey, P. Celliers, C. Cerjan, E. Dewald, T. Dittrich, T. Döppner, D. Hinkel, L. Hopkins, J. Kline, S. Pape, T. Ma, M. a., J. Milovich, a. Pak, H. Park, P. Patel, B. Remington, J. Salmonson, P. Springer, R. Tommasini, Fuel gain exceeding unity in an inertially confined fusion implosion, Nature 506 (2014) 343-348.

[90] G. Gregori, A. Ravasio, C. D. Murphy, K. Schaar, A. Baird, a. R. Bell, A. Benuzzi-Mounaix, R. Bingham, C. Constantin, R. P. Drake, M. Edwards, E. T. Everson, C. D. Gregory, Y. Kuramitsu, W. Lau, J. Mithen, C. Niemann, H.-S. Park, B. a. Remington, B. Reville, a. P. L. Robinson, D. D. Ryutov, Y. Sakawa, S. Yang, N. C. Woolsey, M. Koenig, F. Miniati, Generation of scaled protogalactic seed magnetic fields in laser-produced shock waves, Nature 481 (7382) (2012) 480-483.

[91] M. Koenig, E. Henry, G. Huser, A. Benuzzi-Mounaix, B. Faral, E. Martinolli, S. Lepape, T. Vinci, D. Batani, M. Tomasini, B. Telaro, P. Loubeyre, T. Hall, P. Celliers, G. Collins, L. DaSilva, R. Cauble, D. Hicks, D. Bradley, A. MacKinnon, P. Patel, J. Eggert, J. Pasley, O. Willi, D. Neely, M. Notley, C. Danson, M. Borghesi, L. Romagnani, T. Boehly, K. Lee, High pressures generated by laser driven shocks: applications to planetary physics, Nuclear Fusion 44 (2004) S208.

[92] J. F. Hansen, M. J. Edwards, D. Froula, G. Gregori, A. Edens, T. Ditmire, Laboratory Simulations of Supernova Shockwave Propagation, Ap\&SS 298 (2005) 61-67.

[93] J. F. Hansen, M. J. Edwards, D. H. Froula, G. Gregori, A. D. Edens, T. Ditmire, Laboratory observation of secondary shock formation ahead of a strongly radiative blast wave, Physics of Plasmas 13 (2006) 22105.

[94] G. S. Settles, Schlieren and Shadowgraph Techniques, Springer, 2001.

[95] J. J. Macfarlane, I. E. Golovkin, P. R. Woodruff, HELIOS-CR A 1-D radiation-magnetohydrodynamics code with inline atomic kinetics modeling, Journal of Quantitative Spectroscopy and Radiative Transfer 99 (2006) 381.

[96] E. T. Everson, P. Pribyl, C. G. Constantin, A. Zylstra, D. Schaeffer, N. L. Kugland, C. Niemann, Design, construction, and calibration of a three-axis, high-frequency magnetic probe (B-dot probe) as a diagnostic for exploding plasmas, Review of Scientific Instruments 80 (11) (2009) 113505.

[97] A. Bell, R. Kingham, Resistive Collimation of Electron Beams in Laser-Produced Plasmas, Physical Review Letters 91 (3) (2003) 35003.

[98] F. Miniati, A. R. Bell, Resistive magnetic fields at cosmic dawn, Astrophys. J. 729 (2011) 73.

[99] C. Reich, P. Gibbon, I. USCHMANN, E. Förster, Yield Optimization and Time Structure of Femtosecond Laser Plasma K $\alpha$ Sources, Physical Review Letters 84 (2000) 4846.

[100] M. Borghesi, a. MacKinnon, a. Bell, R. Gaillard, O. Willi, Megagauss Magnetic Field Generation and Plasma Jet Formation on Solid Targets Irradiated by an Ultraintense Picosecond Laser Pulse, Physical Review Letters 81 (1998) 112-115.

[101] R. P. Drake, G. Gregori, Design Considerations for Unmagnetized Collisionless-Shock Measurements in Homologous Flows, Astrophysical Journal 749 (2012) 171

[102] H. K. Moffatt, Magnetic field generation in electrically conducting fluids, Cambridge, England, Cambridge University Press, 1978.

[103] W. D. Hayes, The vorticity jump across a gasdynamic discontinuity, Journal of Fluid Mechanics 2 (1957) 595.

[104] A. Robinson, H. Schmitz, T. Fox, J. Pasley, D. Symes, Vorticity deposition, structure generation and the approach to self- similarity in colliding blast wave experiments, High Energy Density Phys. 14 (2015) 6-12. 
[105] U. Ziegler, A central-constrained transport scheme for ideal magnetohydrodynamics, Journal of Computational Physics 196 (2004) 393-416.

[106] M. Roth, The diagnostics of ultra-short pulse laser-produced plasma, J. Instrum. 6 (2011) R09001.

[107] M. Tatarakis, J. R. Davies, P. Lee, P. A. Norreys, N. G. Kassapakis, F. N. Beg, A. R. Bell, M. G. Haines, A. E. Dangor, Plasma Formation on the Front and Rear of Plastic Targets due to High-Intensity Laser-Generated Fast Electrons, Physical Review Letters 81 (1998) 999-1002.

[108] J. R. Davies, A. R. Bell, M. Tatarakis, Magnetic focusing and trapping of high-intensity laser-generated fast electrons at the rear of solid targets, Phys. Rev. E 59 (1999) 6032-6036.

[109] P. Gibbon, Short pulse laser interactions with matter, Imperial College Press London, 2005.

[110] S. Wilks, W. Kruer, M. Tabak, A. Langdon, ABSORPTION OF ULTRA-INTENSE LASER-PULSES, Phys. Rev. Lett. 69 (1992) 13831386.

[111] I. A. Bush, R. Kingham, A. P. L. Robinson, J. Pasley, Cavitation and shock wave formation in dense plasmas by relativistic electron beams, Plasma Physics and Controlled Fusion 52 (12) (2010) 125007

[112] A. R. Bell, Turbulent amplification of magnetic field and diffusive shock acceleration of cosmic rays, MNRAS353 (2004) 550-558.

[113] A. R. Bell, The interaction of cosmic rays and magnetized plasma, MNRAS358 (2005) 181-187.

[114] B. Reville, A. R. Bell, Universal behaviour of shock precursors in the presence of efficient cosmic ray acceleration, MNRAS430 (2013) 2873-2884. arXiv:1301.3173.

[115] C. Danson, P. Brummitt, R. Clarke, J. Collier, B. Fell, A. Frackiewicz, S. Hancock, S. Hawkes, C. Hernandez-Gomez, P. Holligan, M. Hutchinson, A. Kidd, W. Lester, I. Musgrave, D. Neely, D. Neville, P. Norreys, D. Pepler, C. Reason, W. Shaikh, T. Winstone, R. Wyatt, B. Wyborn, Vulcan petawattan ultra-high-intensity interaction facility, Nuclear Fusion 44 (2004) S239.

[116] D. Strickland, G. Mourou, Compression of amplified chirped optical pulses, Opt. Comm. 55 (1985) 447-449.

[117] N. C. Woolsey, Y. A. Ali, R. G. Evans, R. A. D. Grundy, S. J. Pestehe, P. G. Carolan, N. J. Conway, R. O. Dendy, P. Helander, K. G. McClements, J. G. Kirk, P. A. Norreys, M. M. Notley, S. J. Rose, Collisionless shock and supernova remnant simulations on VULCAN, Physics of Plasmas 8 (2001) 2439-2445

[118] C. Courtois, R. A. D. Grundy, A. D. Ash, D. M. Chambers, N. C. Woolsey, R. O. Dendy, K. G. McClements, Experiment on collisionless plasma interaction with applications to supernova remnant physics, Physics of Plasmas 11 (2004) 3386-3393. doi:10.1063/1.1752930.

[119] W. Fox, G. Fiksel, a. Bhattacharjee, P.-Y. Chang, K. Germaschewski, S. X. Hu, P. M. Nilson, Filamentation Instability of Counterstreaming Laser-Driven Plasmas, Physical Review Letters 111 (2013) 225002.

[120] M. Borghesi, D. H. Campbell, A. Schiavi, M. G. Haines, O. Willi, A. J. MacKinnon, P. Patel, L. A. Gizzi, M. Galimberti, R. J. Clarke, F. Pegoraro, H. Ruhl, S. Bulanov, Electric field detection in laser-plasma interaction experiments via the proton imaging technique, Physics of Plasmas (1994-present) 9 (5).

[121] M. Roth, A. Blazevic, M. Geissel, T. Schlegel, T. E. Cowan, M. Allen, J.-C. Gauthier, P. Audebert, J. Fuchs, J. Meyer-Ter-Vehn, M. Hegelich, S. Karsch, A. Pukhov, Energetic ions generated by laser pulses: A detailed study on target properties, Physical Review Special Topics Accelerators and Beams 5 (2002) 061301.

[122] S. C. Wilks, A. B. Langdon, T. E. Cowan, M. Roth, M. Singh, S. Hatchett, M. H. Key, D. Pennington, A. MacKinnon, R. A. Snavely, Energetic proton generation in ultra-intense laser-solid interactions, Physics of Plasmas (2001) 542-549.

[123] L. Robson, P. T. Simpson, R. J. Clarke, K. W. D. Ledingham, F. Lindau, O. Lundh, T. McCanny, P. Mora, D. Neely, C.-G. Wahlström, M. Zepf, P. McKenna, Scaling of proton acceleration driven by petawatt-laserplasma interactions, Nature Physics 3 (2006) 58-62.

[124] N. L. Kugland, D. D. Ryutov, P.-Y. Chang, R. P. Drake, G. Fiksel, D. H. Froula, S. H. Glenzer, G. Gregori, M. Grosskopf, M. Koenig, Y. Kuramitsu, C. Kuranz, M. C. Levy, E. Liang, J. Meinecke, F. Miniati, T. Morita, A. Pelka, C. Plechaty, R. Presura, A. Ravasio, B. a. Remington, B. Reville, J. S. Ross, Y. Sakawa, A. Spitkovsky, H. Takabe, H.-S. Park, Self-organized electromagnetic field structures in laser-produced counter-streaming plasmas, Nature Physics 8 (2012) 1-4

[125] C. M. Huntington, F. Fiuza, J. S. Ross, A. B. Zylstra, R. P. Drake, D. H. Froula, G. Gregori, N. L. Kugland, C. C. Kuranz, M. C. Levy, C. K. Li, J. Meinecke, T. Morita, R. Petrasso, C. Plechaty, B. A. Remington, D. D. Ryutov, Y. Sakawa, A. Spitkovsky, H. Takabe, H.-S. Park, Observation of magnetic field generation via the Weibel instability in interpenetrating plasma flows, Nature Physics 11 (2015) $173-176$. arXiv: 1310.3337 , doi:10.1038/nphys3178.

[126] R. A. Fonseca, S. F. Martins, L. O. Silva, J. W. Tonge, F. S. Tsung, W. B. Mori, One-to-one direct modeling of experiments and astrophysical scenarios: pushing the envelope on kinetic plasma simulations, Plasma Physics and Controlled Fusion (2008) 124034.

[127] D. A. Frail, E. Berger, T. Galama, S. R. Kulkarni, G. H. Moriarty-Schieven, G. G. Pooley, R. Sari, D. S. Shepherd, G. B. Taylor, F. Walter, The Enigmatic Radio Afterglow of GRB 991216, Astrophysical Journal 538 (2000) L129-L132.

[128] P. Odier, J.-F. Pinton, S. Fauve, Advection of a magnetic field by a turbulent swirling flow, Physical Review E 58 (1998) 7397-7401.

[129] R. Monchaux, M. Berhanu, M. Bourgoin, M. Moulin, P. Odier, J.-F. Pinton, R. Volk, S. Fauve, N. Mordant, F. Pétrélis, A. Chiffaudel, F. Daviaud, B. Dubrulle, C. Gasquet, L. Marié, F. Ravelet, Generation of a Magnetic Field by Dynamo Action in a Turbulent Flow of Liquid Sodium, Physical Review Letters 98 (2007) 044502.

[130] A. A. Schekochihin, A. B. Iskakov, S. C. Cowley, J. C. McWilliams, M. R. E. Proctor, T. A. Yousef, Fluctuation dynamo and turbulent induction at low magnetic Prandtl numbers, New J. Phys. 9 (2007) 300.

[131] G. S. Golitsyn, Fluctuations of the Magnetic Field and Current Density in a Turbulent Flow of a Weakly Conducting Fluid, Soviet Phys. Doklady 5 (1960) 536-539.

[132] H. K. Moffatt, The amplification of a weak applied magnetic field by turbulence in fluids of moderate conductivity, J. Fluid Mech. 11 (1961) $625-635$.

[133] A. Ruzmaikin, A. Shukurov, Spectrum of the galactic magnetic fields, Astrophysics and Space Science 82 (1982) 397-407.

[134] R. M. Kulsrud, S. W. Anderson, The spectrum of random magnetic fields in the mean field dynamo theory of the Galactic magnetic field, ApJ 396 (1992) 606-630. doi:10.1086/171743.

[135] J. MacFarlane, G. I., P. Wang, P. Woodruff, N. Pereyra, Spect3d - a multi-dimensional collisional-radiative code for generating diagnostic signatures based on hydrodynamics and PIC simulation output, High Energy Dens. Phys. 3 (2007) 181-190.

[136] G. Comte-Bellot, S. Corrsin, The use of a contraction to improve the isotropy of grid-generated turbulence, J. Fluid Mech. 25 (1966) 1-26.

[137] A. Dubey, K. Antypas, M. K. Ganapathy, L. B. Reid, K. Riley, D. Sheeler, A. Siegel, K. Weide, Extensible component-based architecture 
for flash, a massively parallel, multiphysics simulation code, Parallel Computing 35 (2009) 512 - 522.

[138] D. Lee, A solution accurate, efficient and stable unsplit staggered mesh scheme for three dimensional magnetohydrodynamics, Journal of Computational Physics 243 (2013) 269-292.

[139] P. Tzeferacos, M. Fatenejad, N. Flocke, G. Gregori, D. Lamb, D. Lee, J. Meinecke, A. Scopatz, K. Weide, Magnetohydrodynamic simulations of shock-generated magnetic field experiments, High Energy Dens. Phys. 8 (2012) 322-328.

[140] P. Tzeferacos, M. Fatenejad, N. Flocke, C. Graziani, G. Gregori, D. Lamb, D. Lee, J. Meinecke, A. Scopatz, K. Weide, Flash mhd simulations of experiments that study shock-generated magnetic fields, High Energy Dens. Phys. (2014) in press.

[141] G. I. Taylor, The Spectrum of Turbulence, Proceedings of the Royal Society A: Mathematical, Physical and Engineering Sciences 164 (1938) 476-490.

[142] J. Meinecke, P. Tzeferacos, A. R. Bell, R. Bingham, R. J. Clarke, E. M. Churazov, R. Crowston, H. Doyle, R. P. Drake, R. Heathcote, M. Koenig, Y. Kuramitsu, C. C. Kuranz, D. Lee, M. J. MacDonald, C. D. Murphy, M. M. Notley, H.-S. Park, A. Pelka, A. Ravasio, B. Reville, Y. Sakawa, W. C. Wan, N. C. Woolsey, R. Yurchak, F. Miniati, A. A. Schekochihin, D. Q. Lamb, G. Gregori, Developed turbulence and non-linear amplification of magnetic fields in laboratory and astrophysical plasmas, submitted.

[143] I. Zhuravleva, E. M. Churazov, a. a. Schekochihin, S. W. Allen, P. Arelavo, A. C. Fabian, W. R. Forman, J. S. Sanders, A. Simionescu, R. Sunyaev, A. Vikhlinin, N. Werner, Turbulent heating in the X-ray brightest galaxy clusters, Nature (2014) in press.

[144] I. Zhuravleva, E. M. Churazov, a. a. Schekochihin, E. T. Lau, D. Nagai, M. Gaspari, S. W. Allen, K. Nelson, I. J. Parrish, the Relation Between Gas Density and Velocity Power Spectra in Galaxy Clusters: Qualitative Treatment and Cosmological Simulations, Astrophys. J. 788 (2014) L13.

[145] A. A. Schekochihin, S. C. Cowley, Turbulence, magnetic fields, and plasma physics in clusters of galaxies, Phys. Plasmas 13 (2006) 056501.

[146] E. G. Zweibel, C. Heiles, Magnetic fields in galaxies and beyond, Nature 385 (1997) 131-136.

[147] S. Cabanes, N. Schaeffer, H.-C. Nataf, Turbulence reduces magnetic diffusivity in a liquid sodium experiment, Phys. Rev. Lett. 113 (2014) 184501 .

[148] J. T. Gosling, Coronal mass ejections: An overview, Washington DC American Geophysical Union Geophysical Monograph Series 99 (1997) 9-16. doi:10.1029/GM099p0009.

[149] R. J. van Weeren, H. J. A. Röttgering, M. Brüggen, M. Hoeft, Particle Acceleration on Megaparsec Scales in a Merging Galaxy Cluster, Science 330 (2010) 347-. arXiv:1010.4306, doi:10.1126/science.1194293.

[150] E. Fermi, On the Origin of the Cosmic Radiation, Physical Review 75 (1949) 1169-1174. doi:10.1103/PhysRev.75.1169.

[151] V. L. Ginzburg, S. I. Syrovatskii, The origin of cosmic rays, Topics in Astrophysics and Space Physics, New York: Gordon and Breach, $1969,1969$.

[152] R. Blandford, D. Eichler, Particle acceleration at astrophysical shocks: A theory of cosmic ray origin, Phys. Reports. 154 (1987) 1-75. doi:10.1016/0370-1573(87)90134-7.

[153] L. O. Drury, An introduction to the theory of diffusive shock acceleration of energetic particles in tenuous plasmas, Reports on Progress in Physics 46 (1983) 973-1027. doi:10.1088/0034-4885/46/8/002.

[154] V. F. Hess, Über Beobachtungen der durchdringenden Strahlung bei sieben Freiballonfahrten, Physikalische Zeitschrift 13 (1912) 10841091.

[155] M. Ackermann, M. Ajello, A. Allafort, L. Baldini, J. Ballet, G. Barbiellini, M. G. Baring, D. Bastieri, K. Bechtol, R. Bellazzini, R. D. Blandford, E. D. Bloom, E. Bonamente, A. W. Borgland, E. Bottacini, T. J. Brandt, J. Bregeon, M. Brigida, P. Bruel, R. Buehler, G. Busetto, S. Buson, G. A. Caliandro, R. A. Cameron, P. A. Caraveo, J. M. Casandjian, C. Cecchi, Ö. Celik, E. Charles, S. Chaty, R. C. G. Chaves, A. Chekhtman, C. C. Cheung, J. Chiang, G. Chiaro, A. N. Cillis, S. Ciprini, R. Claus, J. Cohen-Tanugi, L. R. Cominsky, J. Conrad, S. Corbel, S. Cutini, F. D'Ammando, A. de Angelis, F. de Palma, C. D. Dermer, E. do Couto e Silva, P. S. Drell, A. Drlica-Wagner, L. Falletti, C. Favuzzi, E. C. Ferrara, A. Franckowiak, Y. Fukazawa, S. Funk, P. Fusco, F. Gargano, S. Germani, N. Giglietto, P. Giommi, F. Giordano, M. Giroletti, T. Glanzman, G. Godfrey, I. A. Grenier, M.-H. Grondin, J. E. Grove, S. Guiriec, D. Hadasch, Y. Hanabata, A. K. Harding, M. Hayashida, K. Hayashi, E. Hays, J. W. Hewitt, A. B. Hill, R. E. Hughes, M. S. Jackson, T. Jogler, G. Jóhannesson, A. S. Johnson, T. Kamae, J. Kataoka, J. Katsuta, J. Knödlseder, M. Kuss, J. Lande, S. Larsson, L. Latronico, M. Lemoine-Goumard, F. Longo, F. Loparco, M. N. Lovellette, P. Lubrano, G. M. Madejski, F. Massaro, M. Mayer, M. N. Mazziotta, J. E. McEnery, J. Mehault, P. F. Michelson, R. P. Mignani, W. Mitthumsiri, T. Mizuno, A. A. Moiseev, M. E. Monzani, A. Morselli, I. V. Moskalenko, S. Murgia, T. Nakamori, R. Nemmen, E. Nuss, M. Ohno, T. Ohsugi, N. Omodei, M. Orienti, E. Orlando, J. F. Ormes, D. Paneque, J. S. Perkins, M. Pesce-Rollins, F. Piron, G. Pivato, S. Rainò, R. Rando, M. Razzano, S. Razzaque, A. Reimer, O. Reimer, S. Ritz, C. Romoli, M. SánchezConde, A. Schulz, C. Sgrò, P. E. Simeon, E. J. Siskind, D. A. Smith, G. Spandre, P. Spinelli, F. W. Stecker, A. W. Strong, D. J. Suson, H. Tajima, H. Takahashi, T. Takahashi, T. Tanaka, J. G. Thayer, J. B. Thayer, D. J. Thompson, S. E. Thorsett, L. Tibaldo, O. Tibolla, M. Tinivella, E. Troja, Y. Uchiyama, T. L. Usher, J. Vandenbroucke, V. Vasileiou, G. Vianello, V. Vitale, A. P. Waite, M. Werner, B. L. Winer, K. S. Wood, M. Wood, R. Yamazaki, Z. Yang, S. Zimmer, Detection of the Characteristic Pion-Decay Signature in Supernova Remnants, Science 339 (2013) 807-811. arXiv:1302.3307, doi:10.1126/science.1231160.

[156] L. Gargaté, A. Spitkovsky, Ion Acceleration in Non-relativistic Astrophysical Shocks, ApJ 744 (2012) $67 . \quad$ arXiv:1107.0762, doi:10.1088/0004-637X/744/1/67.

[157] A. R. Bell, K. M. Schure, B. Reville, G. Giacinti, Cosmic-ray acceleration and escape from supernova remnants, MNRAS431 (2013) 415-429. arXiv:1301.7264.

[158] F. Guo, J. Giacalone, The Acceleration of Thermal Protons at Parallel Collisionless Shocks: Three-dimensional Hybrid Simulations, ApJ 773 (2013) 158. arXiv:1303.5174, doi:10.1088/0004-637X/773/2/158

[159] B. Reville, A. R. Bell, G. Gregori, Diffusive shock acceleration at laser-driven shocks: studying cosmic-ray accelerators in the laboratory, New Journal of Physics 15 (1) (2013) 015015. arXiv:1211.3638.

[160] P. Ghavamian, S. J. Schwartz, J. Mitchell, A. Masters, J. M. Laming, Electron-Ion Temperature Equilibration in Collisionless Shocks: The Supernova Remnant-Solar Wind Connection, Space Science Rev. 178 (2013) 633-663. arXiv:1305.6617, doi:10.1007/s11214-013-9999-0.

[161] J. R. Jokipii, Cosmic-Ray Propagation. I. Charged Particles in a Random Magnetic Field, ApJ 146 (1966) 480. doi:10.1086/148912.

[162] J. R. Jokipii, Rate of energy gain and maximum energy in diffusive shock acceleration, ApJ 313 (1987) 842-846. doi:10.1086/165022. 
[163] A. Achterberg, L. Ball, Particle acceleration at superluminal quasi-perpendicular shocks. Application to SN1978K and SN1987A, A\&A 285 (1994) 687-704 